ALEA, Lat. Am. J. Probab. Math. Stat. 15, 703-753 (2018)

\title{
Distributional Itô's Formula and Regularization of Generalized Wiener Functionals
}

\section{Takafumi Amaba and Yoshihiro Ryu}

Fukuoka University,

8-19-1 Nanakuma,

Jônan-ku, Fukuoka, 814-0180, Japan.

E-mail address: fmamaba@fukuoka-u.ac.jp

Ritsumeikan University,

1-1-1 Nojihigashi,

Kusatsu, Shiga, 525-8577, Japan.

E-mail address: gr0095kp@ed.ritsumei.ac.jp

\begin{abstract}
We investigate Bochner integrabilities of generalized Wiener functionals. We further formulate an Itô formula for a diffusion in a distributional setting, and apply it to investigate differentiability-index $s$ and integrability-index $p \geqslant 2$ for which the Bochner integral belongs to $\mathbb{D}_{p}^{s}$.
\end{abstract}

\section{Introduction}

In this paper, we justify the symbol " $\int_{0}^{T} \delta_{y}\left(X_{t}\right) \mathrm{d} t$ " denoting a quantity relating to the local time of a $d$-dimensional diffusion process $X=\left(X_{t}\right)_{t \geqslant 0}$ with $X_{0}$ being deterministic (in multi-dimensional case, we assume $X_{0} \neq y$ ), or more generally, the object " $\int_{0}^{T} \Lambda\left(X_{t}\right) \mathrm{d} t$ " where $\Lambda$ is a distribution. Our diffusion process $X=\left(X_{t}\right)_{t \geqslant 0}$ is assumed to satisfy a $d$-dimensional stochastic differential equation

$$
\mathrm{d} X_{t}=\sigma\left(X_{t}\right) \mathrm{d} w(t)+b\left(X_{t}\right) \mathrm{d} t, \quad X_{0}=x \in \mathbb{R}^{d},
$$

where $w=\left(w^{1}(t), \cdots, w^{d}(t)\right)_{t \geqslant 0}$ is a $d$-dimensional Wiener process with $w(0)=0$. The main conditions on $\sigma=\left(\sigma_{j}^{i}\right)_{1 \leqslant i, j \leqslant d}$ and $b=\left(b^{i}\right)_{1 \leqslant i \leqslant d}$ under which we will work are combinations from the following.

Hypothesis 1.1. (H1) the coefficients $\sigma$ and $b$ are $C^{\infty}$, and have bounded derivatives in all orders $\geqslant 1$.

(H2) $\left(\sigma \sigma^{*}\right)(x)$ is strictly positive, where $x=X_{0}$ and $\sigma^{*}$ is the transposed matrix of $\sigma$.

Received by the editors April 26th, 2017; accepted June 3rd, 2018.

2010 Mathematics Subject Classification. Primary 60H07; Secondary 60J60, 60J35.

Key words and phrases. Malliavin calculus, Hölder continuity of diffusion local times, Distributional Itô's formula, Smoothing effect brought by time-integral.

The research of the first author was supported by JSPS KAKENHI Grant Number $15 \mathrm{~K} 17562$. 
(H3) $\sigma \sigma^{*}$ is uniformly positive definite, i.e., there exists $\lambda>0$ such that

$$
\lambda|\xi|_{\mathbb{R}^{d}}^{2} \leqslant\left\langle\xi,\left(\sigma \sigma^{*}\right)(y) \xi\right\rangle_{\mathbb{R}^{d}} \text { for all } \xi, y \in \mathbb{R}^{d},
$$

where $\langle\bullet, \bullet\rangle_{\mathbb{R}^{d}}$ is the standard inner product on $\mathbb{R}^{d}$, and $|\bullet|_{\mathbb{R}^{d}}=|\bullet|$ is the corresponding norm.

(H4) $\sigma$ and $b$ are bounded.

We further formulate stochastic integrals and an Itô formula in this distributional setting and investigate when the local time belongs to $\mathbb{D}_{2}^{s}$ (the Sobolev space of integrability-index 2 and differentiability-index $s \in \mathbb{R}$ with respect to the Malliavin derivative).

In fact, we will formulate $\int_{0}^{T} \delta_{y}\left(X_{t}\right) \mathrm{d} t$ as a Bochner integral in the space of generalized Wiener functional. We remark here the Bochner integrability seems nontrivial when $y=X_{0}$, since $\delta_{y}\left(X_{t}\right)$ no longer makes sense at $t=0$. On the other hand, the local time is usually formulated as a classical Wiener functional. Hence, once the Bochner integrability is proved, a "smoothing effect" should occur in the Bochner integral $\int_{0}^{T} \delta_{y}\left(X_{t}\right) \mathrm{d} t$, i.e., the differentiability-index for $\int_{0}^{T} \delta_{y}\left(X_{t}\right) \mathrm{d} t$, should be greater than that of $\delta_{y}\left(X_{t}\right)$.

In the case of Brownian motion $X_{t}=w(t)$, everything can be explicitly computed and we can exhibit this phenomenon. Namely, the following is the prototype of this study.

Let $\mathscr{S}^{\prime}\left(\mathbb{R}^{d}\right)$ denote the space of all Schwartz distributions on $\mathbb{R}^{d}$.

Theorem 1.1. Assume $d=1$. Let $\Lambda \in \mathscr{S}^{\prime}(\mathbb{R})$ and $s \in \mathbb{R}$. If $\Lambda(w(T)) \in \mathbb{D}_{2}^{s}$ then the mapping

$$
(0, T] \ni t \mapsto \sqrt{\frac{T}{t}} \Lambda\left(\sqrt{\frac{T}{t}} w(t)\right) \in \mathbb{D}_{2}^{s}
$$

is Bochner integrable in $\mathbb{D}_{2}^{s}$ and we have

$$
\int_{0}^{T} \sqrt{\frac{T}{t}} \Lambda\left(\sqrt{\frac{T}{t}} w(t)\right) \mathrm{d} t \in \mathbb{D}_{2}^{s+1} .
$$

Since it is known that $\delta_{0}(w(t)) \in \mathbb{D}_{2}^{(-1 / 2)-}$ (see Watanabe, 1991), we obtain $\int_{0}^{T} \delta_{0}(w(t)) \mathrm{d} t \in \mathbb{D}_{2}^{(1 / 2)-}$, which agrees with the result by Nualart and Vives (1992) and Watanabe (1994a). The proof of this theorem is due to the chaos computations (which is essentially the same as Nualart and Vives, 1992 but with no approximations of the integrand). When $b=\sigma \sigma^{\prime} / 2$, this computation brings the Hölder-continuity of the local time with respect to space variable. The norm on $\mathbb{D}_{p}^{s}$ will be denoted by $\|\bullet\|_{p, s}$.

Theorem 1.2. Let $d=1$. Assume (H1), (H3) and that the drift-coefficient is given by $b=\sigma \sigma^{\prime} / 2$. Then for each $s<\frac{1}{2}$ and $\beta \in\left(0, \min \left\{\frac{1}{2}-s, 1\right\}\right)$, there exists a constant $c=c(s, \beta)>0$ such that

$$
\left\|\sigma(y) \int_{0}^{1} \delta_{y}\left(X_{t}\right) \mathrm{d} t-\sigma(z) \int_{0}^{1} \delta_{z}\left(X_{t}\right) \mathrm{d} t\right\|_{2, s} \leqslant c|y-z|^{\beta}
$$

for every $y, z \in \mathbb{R}$.

The proof of this theorem seems interesting in its own right. The study of Hölder continuity of local times had been initiated by Trotter (1958, inequalities (2.1) and (2.3)), in which the almost-sure Hölder-continuity of the Brownian local 
time $\{l(t, x): t \geqslant 0, x \in \mathbb{R}\}$ in time-space variable $(t, x)$ was proved (see also Boufoussi and Roynette, 1993). There are a lot of such studies (see, e.g., Liang, 2006, Ait Ouahra et al., 2014, Lou and Ouyang, 2017 and references therein).

Theorem 1.2 implies immediately the following.

Corollary 1.3. Under the conditions in Theorem 1.2, let $p_{t}(x, y)$ be the transition density of $X_{t}$. Then the mapping

$$
\mathbb{R} \ni y \mapsto \sigma(y) \int_{0}^{1} p_{t}(x, y) \mathrm{d} t \in \mathbb{R}
$$

is (globally) $\beta$-Hölder continuous for every $\beta<1$.

The latter half of this paper concerns with an Itô formula in a distributional setting. The classical Itô-Tanaka formula had been extended with several formulations (see Föllmer et al., 1995, Bouleau and Yor, 1981, Wang, 1977/78, Kubo, 1983 and so on). In particular, according to results in Wang (1977/78) and Bouleau and Yor (1981), the Itô-Tanaka formula for $f\left(X_{t}\right)$ is valid in the case where $f$ is just a convex function. In our case, we obtained the following.

Theorem 1.4. Assume (H1), (H2) and (H4). Let $A_{i}=\sum_{j=1}^{d} \sigma_{i}^{j} \partial /\left(\partial x_{j}\right)$ and $L$ be the generator of the diffusion process $X$. Suppose that $f: \mathbb{R}^{d} \rightarrow \mathbb{R}$ is a measurable function such that

(i) $f$ is continuous at $x$,

(ii) $f$ has at most exponential growth,

(iii) $\int_{0}^{T}\left\|\left(A_{i} f\right)\left(X_{t}\right)\right\|_{2,-k}^{2} \mathrm{~d} t<+\infty$ for $i=1,2, \cdots, d$,

(iv) $\int_{0}^{T}\left\|(L f)\left(X_{t}\right)\right\|_{2,-k} \mathrm{~d} t<+\infty$

for some $k \in \mathbb{N}$. Then we have

$$
f\left(X_{T}\right)-f(x)=\sum_{i=1}^{d} \int_{0}^{T}\left(A_{i} f\right)\left(X_{t}\right) \mathrm{d} w^{i}(t)+\int_{0}^{T}(L f)\left(X_{t}\right) \mathrm{d} t \quad \text { in } \mathbb{D}^{-\infty} .
$$

We can drop the assumption (H4) if $f$ has at most polynomial growth.

The definition of stochastic integral will be given in Section 4.1 and the timeintegral $\int_{0}^{T}(L f)\left(X_{t}\right) \mathrm{d} t$ is understood in the sense of Bochner integral in $\mathbb{D}_{2}^{-k}$. Kubo (1983) also obtained an Itô formula for Brownian motion in a distributional setting. However, his formula does not need to consider the Bochner integrability because the time-interval of integration is a closed interval excluding zero. A generalization to the case of one-dimensional fractional Brownian motion was done by Bender (2003) (and see references therein), in which, even the case where the time-interval of integration is such as $(0, T]$ is considered (Bender, 2003, Theorem 4.4), though the first distributional derivative of $f$ is assumed to be a regular distribution. But he did not give a systematic treatment of Bochner integrability. Theorem 1.4 will be proved in Section 4 and it will be established in Section 4.2 even the case where $f$ itself is a distribution of exponential-type and furthermore the time-interval of integration is $(0, T]$.

A distribution $\Lambda \in \mathscr{S}^{\prime}\left(\mathbb{R}^{d}\right)$ is said to be positive if $\langle\Lambda, f\rangle \geqslant 0$ for every nonnegative test function $f \in \mathscr{S}\left(\mathbb{R}^{d}\right)$. To include local times for diffusions in our scope, we prepare the following 
Theorem 1.5. Assume $d=1,(\mathrm{H} 1)$ and $(\mathrm{H} 2)$. Let $\Lambda \in \mathscr{S}^{\prime}(\mathbb{R})$ be positive. Then there exists $k \in \mathbb{Z}_{\geqslant 0}$ such that we have $\int_{0}^{T}\left\|\Lambda\left(X_{t}\right)\right\|_{p,-2 k} \mathrm{~d} t<+\infty$ for every $p \in$ $(1, \infty)$.

Hence the mapping $(0, T] \ni t \mapsto \delta_{y}\left(X_{t}\right) \in \mathbb{D}_{p}^{-2 k}$ is Bochner integrable in the case of $d=1$. For multi-dimensional cases, it is sufficient to assume $x \neq y$ in order to guarantee the Bochner integrability (Proposition 3.12).

Finally, let $H_{p}^{s}\left(\mathbb{R}^{d}\right):=(1-\triangle)^{-s / 2} L_{p}\left(\mathbb{R}^{d}, \mathrm{~d} z\right)$ for $p \in(1, \infty), s \in \mathbb{R}$, which are called the Bessel potential spaces (see Abels, 2012 or Krylov, 2008 for details). We will then apply the Itô formula (Theorem 4.9) to derive the following.

Corollary 1.6. Assume (H1), (H3) and (H4). Let $p \in(1, \infty)$ and $s \in \mathbb{R}$. Then for each $\Lambda \in H_{p}^{s}\left(\mathbb{R}^{d}\right)$, we have

(i) $\Lambda\left(X_{t}\right) \in \mathbb{D}_{p^{\prime}}^{s}$, for $t>0$ and $p^{\prime} \in(1, p)$;

(ii) if $p>2$, we further have $\int_{t_{0}}^{T} \Lambda\left(X_{t}\right) \mathrm{d} t \in \mathbb{D}_{p^{\prime}}^{s+1}$ for $t_{0} \in(0, T]$ and $p^{\prime} \in[2, p)$.

It might be natural to ask about the class to which $\int_{t_{0}}^{T} \Lambda\left(X_{t}\right) \mathrm{d} t$ belongs when $t_{0}=0$. Some examples are included in Section 4.3.

The organization of the current paper is as follows: We first review the classical Malliavin calculus in Section 2 to introduce several notations. In particular, the mapping of Watanabe's pull-back will be extended to the space of distributions of exponential-type. Section 3 is devoted to investigate Bochner integrability of the mapping $(0, T] \ni t \mapsto \Lambda\left(X_{t}\right)$ where $\Lambda$ is a distribution. We will illustrate the Brownian case with detailed computations. The methods there bring a Hölder continuity in the space variable of the local time in the case where the stochastic differential equation is written in a Fisk-Stratonovich symmetric form. In Section 4, we give a definition of stochastic integrals and formulate an Itô formula in this distributional setting. Corollary 1.6 and some examples will be presented in Section 4.3. Several estimates necessary for these examples are wrapped up in Appendix A.

\section{Review of Malliavin Calculus}

First, we make a brief review of the classical Malliavin calculus on the $d$-dimensional classical Wiener space to introduce notations.

Let $(W, \mathcal{F}, \mathbf{P})$ be the $d$-dimensional Wiener space on $[0, T]$, that is, $W$ is the space of all continuous functions $[0, T] \rightarrow \mathbb{R}^{d}, \mathcal{F}$ is the $\sigma$-field generated by the canonical process $W \ni w \mapsto w(t) \in \mathbb{R}^{d}, 0 \leqslant t \leqslant T$, and $\mathbf{P}$ is the Wiener measure with $\mathbf{P}(w(0)=0)=1$. The expectation under $\mathbf{P}$ will be denoted by $\mathbf{E}$. The space $W$ contains the subspace $H$, consisting of all absolutely continuous $h \in W$ with $h(0)=0$ and the square-integrable derivative. The subspace $H$ is called the Cameron-Martin subspace and forms a real Hilbert space with the inner product

$$
\left\langle h_{1}, h_{2}\right\rangle_{H}:=\int_{0}^{T}\left\langle\dot{h}_{1}(t), \dot{h}_{2}(t)\right\rangle_{\mathbb{R}^{d}} \mathrm{~d} t, \quad h_{1}, h_{2} \in H .
$$

It is known that $L_{2}:=L_{2}(W, \mathcal{F}, \mathbf{P})$ has the following orthogonal decomposition, called the Wiener-Itô chaos expansion:

$$
L_{2}=\mathbb{R} \oplus \mathcal{C}_{1} \oplus \mathcal{C}_{2} \oplus \cdots,
$$


where each $\mathcal{C}_{k}$ is a closed linear subspace of $L_{2}$ spanned by multiple stochastic integrals

$$
\int_{0 \leqslant t_{1}<\cdots<t_{k} \leqslant T}\left\langle\dot{h}_{1}\left(t_{1}\right) \otimes \cdots \otimes \dot{h}_{k}\left(t_{k}\right), \mathrm{d} w\left(t_{1}\right) \otimes \cdots \otimes \mathrm{d} w\left(t_{k}\right)\right\rangle_{\left(\mathbb{R}^{d}\right)^{\otimes k}}
$$

for $h_{1}, h_{2}, \cdots, h_{k} \in H$, of $k$-th degree. Each $\mathcal{C}_{k}$ is called the subspace of Wiener's homogeneous chaos of $k$-th order. We denote by $J_{k}$ the orthogonal projection onto $\mathcal{C}_{k}$. For each separable Hilbert space $\left(E,\langle\bullet, \bullet\rangle_{E}\right), L_{p}(E)$ denotes the space of $E$ valued $p$-th order integrable random variables $F$ with norm $\|F\|_{p}=\mathbf{E}\left[|F|_{E}^{p}\right]^{1 / p}$. Each projection $J_{n}$ extends to $L_{2}(E) \cong L_{2} \otimes E \rightarrow \mathcal{C}_{n} \otimes E$, which is still denoted by the same symbol.

For each $s \in \mathbb{R}$ and $p \in(1, \infty)$, a Sobolev-type space $\mathbb{D}_{p}^{s}(E)$ (we write this $\mathbb{D}_{p}^{s}$ when $E=\mathbb{R})$ is defined as the completion of $\mathcal{P}:=\cup_{n=1}^{\infty} \cap_{m} \geqslant n\left\{F \in L_{2}(E): J_{m} F=\right.$ $0\}$ under the norm $\|\cdot\|_{p, s}$ defined by $\|F\|_{p, s}=\left\|(I-\mathcal{L})^{s / 2} F\right\|_{p}$ for $F \in \mathcal{P}$, where $\mathcal{L}$ is the Ornstein-Uhlenbeck operator on the Wiener space. It is known that

$$
(I-\mathcal{L})^{s / 2} F=\sum_{k=0}^{\infty}(1+k)^{s / 2} J_{k} F, \quad F \in \mathcal{P} .
$$

Note that $\mathbb{D}_{p}^{0}=L_{p}$ for $p \in(1, \infty)$, and

$$
\|F\|_{2, s}^{2}=\sum_{k=0}^{\infty}(1+k)^{s}\left\|J_{k} F\right\|_{2}^{2}, \quad F \in \mathbb{D}_{2}^{s} .
$$

We further define

$$
\mathbb{D}^{\infty}(E):=\bigcap_{s>0} \bigcap_{1<p<\infty} \mathbb{D}_{p}^{s}(E) \text { and } \mathbb{D}^{-\infty}(E):=\bigcup_{s<0} \bigcup_{1<p<\infty} \mathbb{D}_{p}^{s}(E) .
$$

It is known that $\left(\mathbb{D}_{p}^{s}(E)\right)^{\prime}=\mathbb{D}_{q}^{-s}(E)$ if and only if $1 / p+1 / q=1$ (where "" stands for the "continuous dual") for each $s \in \mathbb{R}$, the space $\mathbb{D}^{\infty}(E)$ is a complete countably-normed space and $\mathbb{D}^{-\infty}(E)$ is its dual which is called the space of generalized Wiener functionals. The pairing of $\Phi \in \mathbb{D}^{-\infty}(E)$ and $F \in \mathbb{D}^{\infty}(E)$ is written as $\mathbf{E}[\Phi F]:=\mathbb{D}^{-\infty}(E)\langle\Phi, F\rangle_{\mathbb{D}^{\infty}(E)}$, and then $\mathbf{E}[\Psi]=\mathbb{D}^{-\infty}\langle\Psi, 1\rangle_{\mathbb{D} \infty}$ is called the generalized expectation of $\Psi \in \mathbb{D}^{-\infty}$.

One can define a (continuous) linear operator $D: \mathbb{D}^{-\infty}(E) \rightarrow \mathbb{D}^{-\infty}(E \otimes H)$ such that (a) each restriction $D: \mathbb{D}_{p}^{s+1}(E) \rightarrow \mathbb{D}_{p}^{s}(E \otimes H)$ and is continuous for every $s \in \mathbb{R}$ and $p \in(1, \infty)$, and (b) we have $\langle D F, e \otimes h\rangle_{E \otimes H}=\left\langle D_{h} F, e\right\rangle_{E}$ for $e \in E$, $h \in H$ and $F \in \mathcal{P}$, where $D_{h} F$ is given by

$$
\left\langle\left(D_{h} F\right)(w), e\right\rangle_{E}=\lim _{\varepsilon \rightarrow 0} \frac{1}{\varepsilon}\langle F(w+h)-F(w), e\rangle_{E} \quad \text { for } w \in W .
$$

The differential operator $D_{h}$ in (2.3) is well-defined for almost all $w$ because of the so-called Cameron-Martin theorem. There also exists a (continuous) linear operator $D^{*}: \mathbb{D}^{-\infty}(E \otimes H) \rightarrow \mathbb{D}^{-\infty}(E)$ such that $(\mathrm{a})^{*}$ each restriction $D^{*}: \mathbb{D}_{p}^{s+1}(E \otimes H) \rightarrow$ $\mathbb{D}_{p}^{s}(E)$ and is continuous for every $s \in \mathbb{R}$ and $p \in(1, \infty)$, and (b)* we have

$$
D^{*}(G \otimes h)=-D_{h} G+\int_{0}^{T}\langle\dot{h}(t), \mathrm{d} w(t)\rangle_{\mathbb{R}^{d}} G
$$

for $h \in H, G \in \mathbb{D}_{2}^{1}(E)$. These operators are related as follows: For $F, G \in \mathbb{D}_{2}^{1}(E)$ and $h \in H$, it holds that

$$
\mathbf{E}\left[\langle D F, G \otimes h\rangle_{E \otimes H}\right]=\mathbf{E}\left[\left\langle F, D^{*}(G \otimes h)\right\rangle_{E}\right] .
$$


Let $\mathscr{S}\left(\mathbb{R}^{d}\right)$ be the real Schwartz space of rapidly decreasing $C^{\infty}$-functions on $\mathbb{R}^{d}$. We denote by $\mathscr{S}_{2 k}\left(\mathbb{R}^{d}\right), k \in \mathbb{Z}$ the completion of $\mathscr{S}\left(\mathbb{R}^{d}\right)$ by the norm

$$
|\phi|_{2 k}:=\left|\left(1+x^{2}-\triangle / 2\right)^{k} \phi\right|_{\infty}, \quad \phi=\phi(x) \in \mathscr{S}\left(\mathbb{R}^{d}\right),
$$

where $\triangle=\sum_{i=1}^{d} \partial^{2} /\left(\partial x_{i}\right)^{2}$ and $|\phi|_{\infty}=\sup _{x \in \mathbb{R}^{d}}|\phi(x)|$.

Definition 2.1. $\quad$ (i) A Wiener functional $F=\left(F^{1}, \cdots, F^{d}\right) \in \mathbb{D}^{\infty}\left(\mathbb{R}^{d}\right)$ is said to be non-degenerate if $\left\|\operatorname{det}\left(\left\langle D F^{i}, D F^{j}\right\rangle_{H}\right)_{i, j}^{-1}\right\|_{p}<\infty$ for any $p \in(1, \infty)$.

(ii) A family of Wiener functionals $F_{\alpha}=\left(F_{\alpha}^{1}, \cdots, F_{\alpha}^{d}\right) \in \mathbb{D}^{\infty}\left(\mathbb{R}^{d}\right), \alpha \in I$, where $I$ is an index set, is said to be uniformly non-degenerate if for any $p \in(1, \infty)$, it holds that $\sup _{\alpha \in I}\left\|\operatorname{det}\left(\left\langle D F_{\alpha}^{i}, D F_{\alpha}^{j}\right\rangle_{H}\right)_{i, j}^{-1}\right\|_{p}<\infty$.

If $F \in \mathbb{D}^{\infty}\left(\mathbb{R}^{d}\right)$ is non-degenerate, then the mapping $\mathscr{S}\left(\mathbb{R}^{d}\right) \ni \phi \mapsto \phi(F) \in \mathbb{D}^{\infty}$ extends uniquely to a mapping $\mathscr{S}^{\prime}\left(\mathbb{R}^{d}\right) \ni \Lambda \mapsto \Lambda(F) \in \cup_{s>0} \cap_{1<p<\infty} \mathbb{D}_{p}^{-s}$ such that each restriction maps $\mathscr{S}_{-2 k}\left(\mathbb{R}^{d}\right) \rightarrow \mathbb{D}_{p}^{-2 k}$ and is continuous for every $k \in \mathbb{Z}$ and $p \in$ $(1, \infty)$ (see e.g., Ikeda and Watanabe, 1989, Chapter V, Section 9). The generalized Wiener functional $\Lambda(F)$ is called the pull-back of $\Lambda \in \mathscr{S}^{\prime}\left(\mathbb{R}^{d}\right)$ by $F \in \mathbb{D}^{\infty}\left(\mathbb{R}^{d}\right)$.

For $\Lambda \in \mathscr{S}^{\prime}(\mathbb{R})$, we denote by $\Lambda^{(n)}$ the $n$-th distributional derivative of $\Lambda$.

Lemma 2.2. Let $y \in \mathbb{R}$ and $\delta_{y}$ be the Dirac delta-function at $y$. Then $\delta_{y} \in \mathscr{S}_{-2}(\mathbb{R})$, $\delta_{y}^{(2 k)} \in \mathscr{S}_{-2(k+1)}(\mathbb{R})$ for $k \in \mathbb{N}$, and $\sup _{a \in \mathbb{R}}\left|\delta_{a}\right|_{-2}<+\infty$.

Proof: It is well known that $\delta_{y} \in \mathscr{S}_{-2}(\mathbb{R})$ and $\delta_{y}^{(2 k)} \in \mathscr{S}_{-2(k+1)}(\mathbb{R})$ for $k \in \mathbb{N}$ (see Ikeda and Watanabe, 1989, Chapter V, section 9, Lemma 9.1, p.380). It is also known that

$$
\left(\left(1+x^{2}-\triangle / 2\right)^{-1} \delta_{y}\right)(x) \leqslant \frac{1}{2 \pi} \int_{-\infty}^{+\infty} \frac{\mathrm{e}^{i \xi(x-y)}}{\left(1+\frac{\xi^{2}}{2}\right)} \mathrm{d} \xi
$$

for any $x, y \in \mathbb{R}$, from which, we easily conclude that

$$
\sup _{a \in \mathbb{R}}\left|\delta_{a}\right|_{-2} \leqslant \frac{1}{2 \pi} \int_{-\infty}^{+\infty} \frac{\mathrm{d} \xi}{\left(1+\frac{\xi^{2}}{2}\right)}<+\infty
$$

2.1. Slight extension to exponential-type distributions. It will be convenient to extend the pull-back procedure from Schwartz distribution space to the space of all distributions of exponential-type. Let $\partial_{i}:=\partial /\left(\partial x_{i}\right), i=1,2, \cdots, d$.

Definition 2.3 (Hasumi, 1961). We say $\phi \in C^{\infty}\left(\mathbb{R}^{d}\right)$ belongs to $\mathscr{E}\left(\mathbb{R}^{d}\right)$ if for any $p \in \mathbb{Z}_{\geqslant 0}$ and $k_{1}, \cdots, k_{d} \in \mathbb{Z}_{\geqslant 0}$, we have $\sup _{x \in \mathbb{R}}\left|\exp (p|x|) \partial_{1}^{k_{1}} \cdots \partial_{d}^{k_{d}} \phi(x)\right|<+\infty$.

Semi-norms on $\mathscr{E}\left(\mathbb{R}^{d}\right)$, defined by

$$
|\phi|_{p}:=\sup _{\substack{k_{1}, \cdots, k_{d} \in \mathbb{Z}_{\geqslant 0}: \\ 0 \leqslant k_{1}+\cdots+k_{d} \leqslant p}} \sup _{x \in \mathbb{R}^{d}}\left|\exp (p|x|) \partial_{1}^{k_{1}} \cdots \partial_{d}^{k_{d}} \phi(x)\right|, \quad p=0,1,2, \cdots
$$

make $\mathscr{E}\left(\mathbb{R}^{d}\right)$ a locally convex metrizable space and induces continuous inclusions

$$
\mathscr{D}\left(\mathbb{R}^{d}\right) \hookrightarrow \mathscr{E}\left(\mathbb{R}^{d}\right) \hookrightarrow \mathscr{S}\left(\mathbb{R}^{d}\right) \quad \text { and } \quad \mathscr{S}^{\prime}\left(\mathbb{R}^{d}\right) \hookrightarrow \mathscr{E}^{\prime}\left(\mathbb{R}^{d}\right) \hookrightarrow \mathscr{D}^{\prime}\left(\mathbb{R}^{d}\right)
$$

where $\mathscr{D}^{\prime}\left(\mathbb{R}^{d}\right)$ is the space of all distributions on $\mathbb{R}^{d}$ with the test function space $\mathscr{D}\left(\mathbb{R}^{d}\right)$, and $\mathscr{E}^{\prime}\left(\mathbb{R}^{d}\right)$ is the continuous dual of $\mathscr{E}\left(\mathbb{R}^{d}\right)$. Elements in $\mathscr{E}^{\prime}\left(\mathbb{R}^{d}\right)$ are referred as distributions of exponential-type. The following is known (see Hasumi, 1961, Proposition 3). 
Theorem 2.4. For any $\Lambda \in \mathscr{E}^{\prime}\left(\mathbb{R}^{d}\right)$, there exist $k \in \mathbb{Z}_{\geqslant 0}$ and a bounded continuous function $f: \mathbb{R}^{d} \rightarrow \mathbb{R}$ such that

$$
\Lambda=\frac{\partial^{k d}}{\partial x_{1}^{k} \cdots \partial x_{d}^{k}}[\exp (k|x|) f(x)] .
$$

Here, the derivatives are understood in the sense of distributional derivatives.

Remark 2.5. The spaces $\mathscr{E}\left(\mathbb{R}^{d}\right)$ and $\mathscr{E}^{\prime}\left(\mathbb{R}^{d}\right)$ are denoted by $H$ and $\Lambda_{\infty}$ respectively in Hasumi (1961). The space $\mathscr{E}^{\prime}\left(\mathbb{R}^{d}\right)=\Lambda_{\infty}$ is introduced by Sebastião e Silva (1958) in the study of the Fourier transform of $\Lambda_{\infty}$, called ultra-distributions (see Sebastião e Silva, 1958, Hasumi, 1961 and Yoshinaga, 1960).

Define $e_{k}(x):=\prod_{i=1}^{d} \cosh \left(k x_{i}\right)$ for $k \in \mathbb{Z}_{\geqslant 0}$ and $x=\left(x_{1}, \cdots, x_{d}\right) \in \mathbb{R}^{d}$. We postpone the proof of the next proposition after Definition 2.7.

Proposition 2.6. Suppose that $F_{\alpha}=\left(F_{\alpha}^{1}, \cdots, F_{\alpha}^{d}\right) \in \mathbb{D}^{\infty}\left(\mathbb{R}^{d}\right), \alpha \in I$, where $I$ is an index set, are uniformly non-degenerate and satisfy

$$
M_{r}:=\sup _{\alpha \in I} \mathbf{E}\left[\exp \left(r\left|F_{\alpha}\right|_{\mathbb{R}^{d}}\right)\right]<+\infty \quad \text { for each } r>0 .
$$

Then for any $p \in(1, \infty), k \in \mathbb{Z}_{\geqslant 0}$ and $r>0$, there exists a constant $c=$ $c\left(q, k, r, M_{r}\right)>0$, where $1 / p+1 / q=1$, such that

$$
\left\|\frac{\partial^{k d}\left(e_{r} \phi\right)}{\partial x_{1}^{k} \cdots \partial x_{d}^{k}}\left(F_{\alpha}\right)\right\|_{p,-k d} \leqslant c \sup _{x \in \mathbb{R}^{d}}|\phi(x)|
$$

for all $\phi \in \mathscr{S}\left(\mathbb{R}^{d}\right)$ and $\alpha \in I$.

Now, let $F \in \mathbb{D}^{\infty}\left(\mathbb{R}^{d}\right)$ be non-degenerate. Take $\Lambda=\partial_{1}^{k} \cdots \partial_{d}^{k}[\exp (k|x|) f(x)]$ $\in \mathscr{E}^{\prime}\left(\mathbb{R}^{d}\right)$ (where $f$ is the one associated to $\Lambda$ in Theorem 2.4) and assume $k \geqslant 1$. Let $\varepsilon \in(0,1)$ be arbitrary and put $r:=k+\varepsilon>0$. Define $\phi \in C_{0}\left(\mathbb{R}^{d}\right)$ (the space of continuous functions on $\mathbb{R}^{d}$ vanishing at infinity) by $\phi(x):=\left(\mathrm{e}^{k|x|} / \prod_{i=1}^{d} \cosh \left(r x_{i}\right)\right) f(x)$, so that now we have $\Lambda=\partial_{1}^{k} \cdots \partial_{d}^{k}\left(e_{r} \phi\right)=\partial_{1}^{k} \cdots \partial_{d}^{k}\left[\left(\prod_{i=1}^{d} \cosh \left(r x_{i}\right)\right) \phi(x)\right]$. Take any sequence $\phi_{n} \in \mathscr{S}\left(\mathbb{R}^{d}\right), n \in \mathbb{N}$ such that $\left|\phi_{n}-\phi\right|_{\infty} \rightarrow 0$. Then Proposition 2.6 tells us that $\lim _{n \rightarrow \infty}\left[\partial_{1}^{k} \cdots \partial_{d}^{k}\left(e_{r} \phi_{n}\right)\right](F)$ exists in $\mathbb{D}_{p}^{-k d}$ for each $p \in(1, \infty)$. The limit does not depend on the choice of $\varepsilon>0$ and the sequence $\phi_{n} \in \mathscr{S}\left(\mathbb{R}^{d}\right)$. Under these notations, we put the following.

Definition 2.7. We denote the limit by $\Lambda(F)$ and call the pull-back of $\Lambda \in \mathscr{E}^{\prime}\left(\mathbb{R}^{d}\right)$ by $F$.

Proof of Proposition 2.6: Let $p \in(1, \infty), k \in \mathbb{Z}_{\geqslant 0}, r>0, \alpha \in I, \phi \in \mathscr{S}\left(\mathbb{R}^{d}\right)$ and $J \in \mathbb{D}^{\infty}$ be arbitrary. Then

$$
\mathbf{E}\left[\left(\partial_{1}^{k} \cdots \partial_{d}^{k}\left(e_{r} \phi\right)\right)\left(F_{\alpha}\right) J\right]=\mathbf{E}\left[\left(\prod_{i=1}^{d} \cosh \left(r F_{\alpha}^{i}\right)\right) \phi\left(F_{\alpha}\right) l_{\alpha}(J)\right]
$$

where $l_{\alpha}(J) \in \mathbb{D}^{\infty}$ is of the form

$$
l_{\alpha}(J)=\sum_{j=0}^{k d}\left\langle P_{j}(w), D^{j} J\right\rangle_{H^{\otimes j}}
$$

for some $P_{j}(w) \in \mathbb{D}^{\infty}\left(H^{\otimes j}\right), j=0,1, \cdots, k d$ which are polynomials in $F_{\alpha}$, its derivatives up to the order $k d$, and $\operatorname{det}\left(\left\langle D F_{\alpha}^{i}, D F_{\alpha}^{j}\right\rangle_{H}\right)_{i j}^{-1}$. Take $q^{\prime} \in(1, q)$, where 
$1 / p+1 / q=1$. Since $\left\{F_{\alpha}\right\}_{\alpha \in I}$ is uniformly non-degenerate, there exists $c_{0}>0$ such that

$$
\left\|l_{\alpha}(J)\right\|_{q^{\prime}} \leqslant c_{0}\|J\|_{q, k} \text { for all } \alpha \in I \text { and } J \in \mathbb{D}^{\infty} .
$$

Therefore by taking $p^{\prime} \in(1, \infty)$ such that $1 / p^{\prime}+1 / q^{\prime}=1$, we have

$$
\left|\mathbf{E}\left[\left(\prod_{i=1}^{d} \cosh \left(r F_{\alpha}^{i}\right)\right) \phi\left(F_{\alpha}\right) l_{\alpha}(J)\right]\right| \leqslant c_{0}^{\prime}|\phi|_{\infty}\left\|\exp \left(r\left|F_{\alpha}\right|\right)\right\|_{p^{\prime}}\|J\|_{q, k},
$$

for some constant $c_{0}^{\prime}>0$, which implies

$$
\left\|\left(e_{k} \phi\right)^{(k)}\left(F_{\alpha}\right)\right\|_{p,-k} \leqslant c_{0}^{\prime}|\phi|_{\infty}\left\|\exp \left(r\left|F_{\alpha}\right|\right)\right\|_{p^{\prime}} \leqslant c_{0}^{\prime} \sup _{\alpha \in I}\left\|\exp \left(r\left|F_{\alpha}\right|\right)\right\|_{p^{\prime}}|\phi|_{\infty} .
$$

Corollary 2.8. Suppose that $F_{\alpha} \in \mathbb{D}^{\infty}\left(\mathbb{R}^{d}\right)$ for $\alpha \in I \subset \mathbb{R}$, where $I$ is an index set, satisfy

(i) $\left\{F_{\alpha}\right\}_{\alpha \in I}$ is uniformly non-degenerate;

(ii) $\sup _{\alpha \in I} \mathbf{E}\left[\exp \left(r\left|F_{\alpha}\right|_{\mathbb{R}^{d}}\right)\right]<+\infty$ for each $r>0$;

(iii) the mapping $I \ni \alpha \mapsto F_{\alpha} \in \mathbb{D}^{\infty}\left(\mathbb{R}^{d}\right)$ is continuous.

Then for any $p \in(1, \infty)$ and $\Lambda=\partial_{1}^{k} \cdots \partial_{d}^{k}[\exp (k|x|) f(x)] \in \mathscr{E}^{\prime}\left(\mathbb{R}^{d}\right)$ ( $f$ is the one associated to $\Lambda$ in Theorem 2.4), the mapping

$$
I \ni \alpha \mapsto \Lambda\left(F_{\alpha}\right) \in \mathbb{D}_{p}^{-k d}
$$

is continuous.

Proof: Let $p \in(1, \infty), \alpha \in I$ and $\varepsilon>0$ be arbitrary. Suppose that $\Lambda=$ $\partial_{1}^{k} \cdots \partial_{d}^{k}\left[\left(\prod_{i=1}^{d} \cosh \left(r x_{i}\right)\right) \phi(x)\right]$ where $r>k \geqslant 1$ and $\phi \in C_{0}\left(\mathbb{R}^{d}\right)$. Then by Proposition 2.6, there exists $\psi \in \mathscr{S}\left(\mathbb{R}^{d}\right)$ such that $\left\|\Lambda\left(F_{\beta}\right)-\left[\partial_{1}^{k} \cdots \partial_{d}^{k}\left(e_{r} \psi\right)\right]\left(F_{\beta}\right)\right\|_{p,-k}<\varepsilon / 3$ for every $\beta \in I$. Furthermore, by the condition (ii) and (iii), there exists $\delta>0$ such that $\left\|\left[\partial_{1}^{k} \cdots \partial_{d}^{k}\left(e_{r} \psi\right)\right]\left(F_{\beta}\right)-\left[\partial_{1}^{k} \cdots \partial_{d}^{k}\left(e_{r} \psi\right)\right]\left(F_{\alpha}\right)\right\|_{p}<\varepsilon / 3$ if $|\beta-\alpha|<\delta$. Hence, for each $\beta \in I$ with $|\beta-\alpha|<\delta$,

$$
\begin{aligned}
& \left\|\Lambda\left(F_{\alpha}\right)-\Lambda\left(F_{\beta}\right)\right\|_{p,-k} \\
& \begin{array}{r}
\leqslant\left\|\left(F_{\alpha}\right)-\left[\partial_{1}^{k} \cdots \partial_{d}^{k}\left(e_{r} \psi\right)\right]\left(F_{\alpha}\right)\right\|_{p,-k} \\
+\left\|\left[\partial_{1}^{k} \cdots \partial_{d}^{k}\left(e_{r} \psi\right)\right]\left(F_{\alpha}\right)-\left[\partial_{1}^{k} \cdots \partial_{d}^{k}\left(e_{r} \psi\right)\right]\left(F_{\beta}\right)\right\|_{p} \\
\\
\quad+\left\|\left[\partial_{1}^{k} \cdots \partial_{d}^{k}\left(e_{r} \psi\right)\right]\left(F_{\beta}\right)-\Lambda\left(F_{\beta}\right)\right\|_{p,-k}
\end{array} \\
& <\varepsilon .
\end{aligned}
$$

The case of $k=0$ is clear.

If we assume (H1), (H2) and (H4), then we have the Gaussian estimate for the transition density of $X$ (the special case $n=0$ in Lemma 3.10-(ii)) and

$$
\sup _{t \in K} \mathbf{E}[\exp (r|X(t, x, w)|)]<+\infty
$$

for all $r>0$ and compact set $K \subset(0, \infty)$. Hence by Corollary 2.8, for any $\Lambda=$ $\partial_{1}^{k} \cdots \partial_{d}^{k}[\exp (k|x|) f(x)] \in \mathscr{E}^{\prime}\left(\mathbb{R}^{d}\right)$, the mapping $(0, \infty) \ni t \mapsto \Lambda(X(t, x, w)) \in \mathbb{D}_{p}^{-k d}$ is continuous for every $p \in(1, \infty)$. 


\section{Bochner Integrability of Pull-Backs by Diffusions}

Let $w=(w(t))_{t \geqslant 0}$ be a $d$-dimensional Wiener process with $w(0)=0$. Let $\sigma: \mathbb{R}^{d} \rightarrow \mathbb{R}^{d} \otimes \mathbb{R}^{d}, b: \mathbb{R}^{d} \rightarrow \mathbb{R}^{d}$. We consider the following stochastic differential equation

$$
\mathrm{d} X_{t}=\sigma\left(X_{t}\right) \mathrm{d} w(t)+b\left(X_{t}\right) \mathrm{d} t, \quad X_{0}=x \in \mathbb{R}^{d} .
$$

In this section, we assume conditions (H1) and (H2). Under these conditions, the equation (3.1) admits a unique strong solution. We denote by $\{X(t, x, w)\}_{t \geqslant 0}$ the unique strong solution $X=\left(X_{t}\right)_{t \geqslant 0}$ to (3.1). Furthermore, for each $t>0$ and $x \in \mathbb{R}$, we have $X(t, x, w) \in \mathbb{D}^{\infty}\left(\mathbb{R}^{d}\right)$ and is non-degenerate. Henceforward for $t>0$, one can define the pull-back $\Lambda(X(t, x, w))$ of $\Lambda \in \mathscr{S}^{\prime}\left(\mathbb{R}^{d}\right)$ by $X(t, x, w)$ as an element of $\cup_{s>0} \cap_{1<p<\infty} \mathbb{D}_{p}^{-s}$ (Recall things just after Definition 2.1).

Fix $T>0$ be arbitrary. By the condition (H2), we further know that

$$
\sup _{t \in K}\left\|\operatorname{det}\left(\left\langle D X_{t}^{i}, D X_{t}^{j}\right\rangle_{H}\right)_{i j}^{-1}\right\|_{p}<+\infty \quad \text { for } 1<p<\infty
$$

holds for each closed interval $K \subset(0, T]$, which implies that for each $k \in \mathbb{Z}_{\geqslant 0}$, $p \in(1, \infty)$ and $\Lambda \in \mathscr{S}_{-2 k}\left(\mathbb{R}^{d}\right)$, the mapping $(0, T] \ni t \mapsto \Lambda(X(t, x, w)) \in \mathbb{D}_{p}^{-2 k}$ is continuous (see e.g. Watanabe, 1987, Remark 2.2). In particular, the mapping $\left[t_{0}, T\right] \ni t \mapsto \Lambda(X(t, x, w)) \in \mathbb{D}_{p}^{-2 k}$ is Bochner integrable for each $t_{0}>0$, and hence the Bochner integral $\int_{t_{0}}^{T} \Lambda(X(t, x, w)) \mathrm{d} t$ makes sense as an element in $\mathbb{D}_{p}^{-2 k}$ for each $t_{0}>0$. If we assume (H4) additionally, then analogous results follow also for $\Lambda \in \mathscr{E}^{\prime}\left(\mathbb{R}^{d}\right)$ by virtue of the Gaussian estimates for the transition density function of $\left(X_{t}\right)_{t \geqslant 0}$ (or one can refer Lemma 3.10 below) and Corollary 2.8.

Now our problem is the Bochner integrability on $(0, T]$, i.e., whether it holds that $\int_{0}^{T}\|\Lambda(X(t, x, w))\|_{p,-2 k} \mathrm{~d} t<+\infty$ or not. The main results of this section are Theorem 3.4 and Proposition 3.12. Before seeing this, we shall start with the Brownian case, which would be an introductory example.

\subsection{Bochner integrability of $\delta_{0}(w(t))$.}

Proposition 3.1. Let $d=1, \Lambda \in \mathscr{S}^{\prime}(\mathbb{R})$ and $s \in \mathbb{R}$. If $\Lambda(w(T)) \in \mathbb{D}_{2}^{s}$ then the mapping

$$
(0, T] \ni t \mapsto \sqrt{\frac{T}{t}} \Lambda\left(\sqrt{\frac{T}{t}} w(t)\right) \in \mathbb{D}_{2}^{s}
$$

is Bochner integrable in $\mathbb{D}_{2}^{s}$ and we have

$$
\int_{0}^{T} \sqrt{\frac{T}{t}} \Lambda\left(\sqrt{\frac{T}{t}} w(t)\right) \mathrm{d} t \in \mathbb{D}_{2}^{s+1}
$$

Remark 3.2. Hence the Bochner integral poses a sort of "smoothing effect", in the sense of raising the regularity-index $s$, which might be a common understanding for most of us.

Proof: Recall the integration by parts formula

$$
\mathbf{E}\left[\Lambda^{\prime}(w(t)) H_{n}\left(\frac{w(t)}{\sqrt{t}}\right)\right]=t^{-1 / 2} \mathbf{E}\left[\Lambda(w(t)) H_{n+1}\left(\frac{w(t)}{\sqrt{t}}\right)\right]
$$

where $\Lambda^{\prime}=\partial \Lambda$ is the distributional derivative of $\Lambda, H_{n}:=\partial^{* n} 1$ is the $n$-th Hermite polynomial and $\partial^{*}=-\partial+x$. The family $\left\{\frac{1}{\sqrt{n !}} H_{n}\right\}_{n=0}^{\infty}$ forms a complete orthonormal base of $L_{2}\left(\mathbb{R},(2 \pi)^{-1 / 2} \mathrm{e}^{-x^{2} / 2} \mathrm{~d} x\right)$ (see Lemma A.1 in Appendix A). 
Then $\Lambda\left((T / t)^{1 / 2} w(t)\right)$ has the Fourier expansion (the Wiener-Itô chaos expansion)

$$
\Lambda\left(\sqrt{\frac{T}{t}} w(t)\right)=\sum_{n=0}^{\infty} \frac{1}{n !} \mathbf{E}\left[\Lambda\left(\sqrt{\frac{T}{t}} w(t)\right) H_{n}\left(\frac{w(t)}{\sqrt{t}}\right)\right] H_{n}\left(\frac{w(t)}{\sqrt{t}}\right),
$$

and hence

$$
\left\|\Lambda\left(\sqrt{\frac{T}{t}} w(t)\right)\right\|_{2, s}^{2}=\sum_{n=0}^{\infty} \frac{(1+n)^{s}}{n !} \mathbf{E}\left[\Lambda\left(\sqrt{\frac{T}{t}} w(t)\right) H_{n}\left(\frac{w(t)}{\sqrt{t}}\right)\right]^{2}
$$

Here we have

$$
\mathbf{E}\left[\Lambda\left(\sqrt{\frac{T}{t}} w(t)\right) H_{n}\left(\frac{w(t)}{\sqrt{t}}\right)\right]=\mathbf{E}\left[\Lambda(w(T)) H_{n}\left(\frac{w(T)}{\sqrt{T}}\right)\right] .
$$

Therefore

$$
\begin{aligned}
& \left\|\sqrt{\frac{T}{t}} \Lambda\left(\sqrt{\frac{T}{t}} w(t)\right)\right\|_{2, s}^{2} \\
& =\frac{T}{t} \sum_{n=0}^{\infty} \frac{(1+n)^{s}}{n !} \mathbf{E}\left[\Lambda(w(T)) H_{n}\left(\frac{w(T)}{\sqrt{T}}\right)\right]^{2}=\frac{T}{t}\|\Lambda(w(T))\|_{2, s}^{2},
\end{aligned}
$$

that is, we get $\left\|(T / t)^{1 / 2} \Lambda\left((T / t)^{1 / 2} w(t)\right)\right\|_{2, s}=(T / t)^{1 / 2}\|\Lambda(w(T))\|_{2, s}$. Since

$$
\int_{0}^{T}\left\|\sqrt{\frac{T}{t}} \Lambda\left(\sqrt{\frac{T}{t}} w(t)\right)\right\|_{2, s} \mathrm{~d} t=T^{1 / 2}\|\Lambda(w(T))\|_{2, s} \int_{0}^{T} t^{-1 / 2} \mathrm{~d} t<+\infty,
$$

the function $(0, T] \ni t \mapsto(T / t)^{1 / 2} \Lambda\left((T / t)^{1 / 2} w(t)\right) \in \mathbb{D}_{2}^{s}$ is Bochner integrable and $\int_{0}^{T}(T / t)^{1 / 2} \Lambda\left((T / t)^{1 / 2} w(t)\right) \mathrm{d} t \in \mathbb{D}_{2}^{s}$.

Next we show

$$
\int_{0}^{T} \sqrt{\frac{T}{t}} \Lambda\left(\sqrt{\frac{T}{t}} w(t)\right) \mathrm{d} t \in \mathbb{D}_{2}^{s+1} .
$$

For this, we note that

$$
\begin{aligned}
& \int_{0}^{T} \sqrt{\frac{T}{t}} \Lambda\left(\sqrt{\frac{T}{t}} w(t)\right) \mathrm{d} t \\
& =\sum_{n=0}^{\infty} \frac{1}{n !} \mathbf{E}\left[\Lambda(w(T)) H_{n}\left(\frac{w(T)}{\sqrt{T}}\right)\right] \int_{0}^{T} \sqrt{\frac{T}{t}} H_{n}\left(\frac{w(t)}{\sqrt{t}}\right) \mathrm{d} t
\end{aligned}
$$

is the chaos expansion for $\int_{0}^{T}(T / t)^{1 / 2} \Lambda\left((T / t)^{1 / 2} w(t)\right) \mathrm{d} t$ (more precisely, we have used Corollary 3.6 below). We shall focus on the $L_{2}$-norm of the last factor:

$$
\begin{aligned}
& \mathbf{E}\left[\left\{\int_{0}^{T} \frac{1}{\sqrt{t}} H_{n}\left(\frac{w(t)}{\sqrt{t}}\right) \mathrm{d} t\right\}^{2}\right] \\
& =2 \int_{0<t<s<T} \frac{1}{\sqrt{t s}} \mathbf{E}\left[H_{n}\left(\frac{w(t)}{\sqrt{t}}\right) H_{n}\left(\frac{w(s)}{\sqrt{s}}\right)\right] \mathrm{d} t \mathrm{~d} s \\
& =2 \int_{0<t<s<T} \frac{1}{\sqrt{t s}} \mathbf{E}\left[H_{n}\left(\frac{w(t)}{\sqrt{t}}\right) H_{n}\left(\sqrt{\frac{t}{s}} \frac{w(t)}{\sqrt{t}}+\frac{w(s)-w(t)}{\sqrt{s}}\right)\right] \mathrm{d} t \mathrm{~d} s \\
& =2 \int_{0<t<s<T} \frac{1}{\sqrt{t s}} n !\left(\frac{t}{s}\right)^{n / 2} \mathrm{~d} t \mathrm{~d} s \\
& =2(n !) \int_{0<t<s<T} t^{(n-1) / 2} s^{-(n+1) / 2} \mathrm{~d} t \mathrm{~d} s=\frac{4 T}{n+1} n ! .
\end{aligned}
$$


In the third equality, we have used the integration by parts formula and $H_{n}^{\prime}=$ $n H_{n-1}$. Hence we have

$$
\begin{aligned}
& \left\|\int_{0}^{T} \sqrt{\frac{T}{t}} \Lambda\left(\sqrt{\frac{T}{t}} w(t)\right) \mathrm{d} t\right\|_{2, s+1}^{2} \\
& =T \sum_{n=0}^{\infty} \frac{(1+n)^{s+1}}{(n !)^{2}} \mathbf{E}\left[\Lambda(w(T)) H_{n}\left(\frac{w(T)}{\sqrt{T}}\right)\right]^{2} \mathbf{E}\left[\left\{\int_{0}^{T} \frac{1}{\sqrt{t}} H_{n}\left(\frac{w(t)}{\sqrt{t}}\right) \mathrm{d} t\right\}^{2}\right] \\
& =4 T^{2} \sum_{n=0}^{\infty} \frac{(1+n)^{s}}{n !} \mathbf{E}\left[\Lambda(w(T)) H_{n}\left(\frac{w(T)}{\sqrt{T}}\right)\right]^{2}=4 T^{2}\|\Lambda(w(T))\|_{2, s}^{2}<+\infty,
\end{aligned}
$$

which proves (3.2).

By Nualart and Vives (1992, Section 2) and Watanabe (1994a), it is known that

$$
\delta_{0}(w(t)) \in \mathbb{D}_{2}^{(-1 / 2)-} \quad \text { but } \quad \delta_{0}(w(t)) \notin \mathbb{D}_{2}^{-1 / 2} \text { for } t>0,
$$

where $\mathbb{D}_{2}^{s-}:=\cap_{\alpha<s} \mathbb{D}_{2}^{\alpha}$. From this fact and Proposition 3.1, we reached the following result by Nualart and Vives (1992) and Watanabe (1994a).

Corollary 3.3. If $d=1$, we have $\int_{0}^{T} \delta_{0}(w(t)) \mathrm{d} t \in \mathbb{D}_{2}^{(1 / 2)-}$.

3.2. Bochner integrability of $\Lambda\left(X_{t}\right)$ where $\Lambda$ is a distribution. A distribution $\Lambda \in$ $\mathscr{D}^{\prime}\left(\mathbb{R}^{d}\right)$ is said to be positive if $\langle\Lambda, f\rangle \geqslant 0$ for all nonnegative $f \in \mathscr{D}\left(\mathbb{R}^{d}\right)$. It is known that for a positive distribution $\Lambda \in \mathscr{D}^{\prime}\left(\mathbb{R}^{d}\right)$, there exists a Radon measure $\mu$ on $\mathbb{R}^{d}$ such that

$$
\langle\Lambda, f\rangle=\int_{\mathbb{R}^{d}}\left\langle\delta_{y}, f\right\rangle \mu(\mathrm{d} y), \quad f \in \mathscr{D}\left(\mathbb{R}^{d}\right) .
$$

The main objective in this section is to prove

Theorem 3.4. Let $d=1$ and $x \in \mathbb{R}$. Suppose that $\Lambda \in \mathscr{D}^{\prime}(\mathbb{R})$ is positive.

(i) If (H1), (H2) and $\Lambda \in \mathscr{S}_{-2 k}(\mathbb{R})$ where $k \in \mathbb{Z}_{\geqslant 0}$, then for any $p \in(1,+\infty)$, $\int_{0}^{T}\|\Lambda(X(t, x, w))\|_{p,-2 k} \mathrm{~d} t<+\infty$.

(ii) If (H1), (H2), (H4) and $\Lambda \in \mathscr{E}^{\prime}(\mathbb{R})$ hold, then

$$
\int_{0}^{T} \int_{\mathbb{R}}\left\|\delta_{y}(X(t, x, w))\right\|_{p,-2} \mu(\mathrm{d} y) \mathrm{d} t<+\infty,
$$

where $\mu$ is the Radon measure associated to $\Lambda$ as above.

Remark 3.5. (a) Therefore if we assume (H1), (H2) and $\Lambda \in \mathscr{S}_{-2 k}(\mathbb{R})$ is positive, then $(0, T] \ni t \mapsto \Lambda\left(X_{t}\right) \in \mathbb{D}_{p}^{-2 k}$ is Bochner integrable. If we have (H4) additionally, the mapping $(0, T] \times \mathbb{R} \ni(t, y) \mapsto \delta_{y}\left(X_{t}\right) \in \mathbb{D}_{p}^{-2}$ is also Bochner integrable with respect to $\mathrm{d} t \otimes \mu(\mathrm{d} y)$ (the measurability can be checked from the construction of $\left.\delta_{y}\left(X_{t}\right)\right)$, and we have

$$
\begin{aligned}
\int_{0}^{T} \Lambda\left(X_{t}\right) \mathrm{d} t & =\int_{0}^{T} \int_{\mathbb{R}} \delta_{y}\left(X_{t}\right) \mu(\mathrm{d} y) \mathrm{d} t \\
& =\int_{\mathbb{R}} \int_{0}^{T} \delta_{y}\left(X_{t}\right) \mathrm{d} t \mu(\mathrm{d} y) \quad \text { in } \mathbb{D}_{p}^{-2} .
\end{aligned}
$$


(b) Under (H1) and (H2), Lemma 2.2 and Theorem 3.4-(i) assures $\int_{0}^{T}\left\|\delta_{y}\left(X_{t}\right)\right\|_{p,-2} \mathrm{~d} t$ is finite for any $y \in \mathbb{R}$.

Corollary 3.6. Let $d=1$. Assume (H1) and (H2). For each $y \in \mathbb{R}$ and $n \in \mathbb{Z}_{\geqslant 0}$, the mapping $(0, T] \ni t \mapsto J_{n}\left[\delta_{y}\left(X_{t}\right)\right] \in L_{2}$ is Bochner integrable and

$$
J_{n}\left[\int_{0}^{T} \delta_{y}\left(X_{t}\right) \mathrm{d} t\right]=\int_{0}^{T} J_{n}\left[\delta_{y}\left(X_{t}\right)\right] \mathrm{d} t .
$$

Proof of Corollary 3.6: By Theorem 3.4, we have

$$
\begin{aligned}
\int_{0}^{T}\left\|J_{n}\left[\delta_{y}\left(X_{t}\right)\right]\right\|_{L_{2}} \mathrm{~d} t & =(1+n) \int_{0}^{T}\left\|J_{n}\left[\delta_{y}\left(X_{t}\right)\right]\right\|_{2,-2} \mathrm{~d} t \\
& \leqslant(1+n) \int_{0}^{T}\left\|\delta_{y}\left(X_{t}\right)\right\|_{2,-2} \mathrm{~d} t<+\infty
\end{aligned}
$$

This shows the Bochner integrability of the mapping $(0, T] \ni t \mapsto J_{n}\left[\delta_{y}\left(X_{t}\right)\right] \in L_{2}$, and hence $\int_{0}^{T} J_{n}\left[\delta_{y}\left(X_{t}\right)\right] \mathrm{d} t \in L_{2}$.

Second, for each $F \in \mathbb{D}^{\infty}$, the mapping $\mathbb{D}_{2}^{s} \ni G \mapsto \mathbf{E}[G F] \in \mathbb{R}$ is linear and bounded for each $s \in \mathbb{R}$. Therefore Bochner integrals and (generalized) expectations may be interchanged, and accordingly we have

$$
\begin{aligned}
& \mathbf{E}\left[J_{n}\left[\int_{0}^{T} \delta_{y}\left(X_{t}\right) \mathrm{d} t\right] F\right]=\mathbf{E}\left[\int_{0}^{T} \delta_{y}\left(X_{t}\right) \mathrm{d} t J_{n} F\right] \\
& =\int_{0}^{T} \mathbf{E}\left[\delta_{y}\left(X_{t}\right) J_{n} F\right] \mathrm{d} t \\
& =\int_{0}^{T} \mathbf{E}\left[J_{n}\left[\delta_{y}\left(X_{t}\right)\right] F\right] \mathrm{d} t=\mathbf{E}\left[\int_{0}^{T} J_{n}\left[\delta_{y}\left(X_{t}\right)\right] \mathrm{d} t F\right],
\end{aligned}
$$

which proves the second assertion.

To prove Theorem 3.4, we need several implements.

For each $\varepsilon>0$, we consider the following $d$-dimensional stochastic differential equation

$$
\mathrm{d} X_{t}=\varepsilon \sigma\left(X_{t}\right) \mathrm{d} w(t)+\varepsilon^{2} b\left(X_{t}\right) \mathrm{d} t .
$$

Similarly to the equation (3.1), we denote by $\left\{X^{\varepsilon}(t, x, w)\right\}_{t \geqslant 0}$ a unique strong solution $X^{\varepsilon}=\left(X_{t}^{\varepsilon}\right)_{t \geqslant 0}$ to (3.4) such that $X_{0}^{\varepsilon}=x \in \mathbb{R}^{d}$. Then it holds that for each $\varepsilon>0,\left\{X\left(\varepsilon^{2} t, x, w\right)\right\}_{t \geqslant 0}$ is equivalent to $\left\{X^{\varepsilon}(t, x, w)\right\}_{t \geqslant 0}$ in law. A more tricky fact which we need is the following.

Proposition 3.7. Let $\Lambda \in \mathscr{S}^{\prime}\left(\mathbb{R}^{d}\right)$. Then for every $p \in(1, \infty), s \in \mathbb{R}$ and $t>0$, we have $\left\|\Lambda\left(X\left(\varepsilon^{2} t, x, w\right)\right)\right\|_{p, s}=\left\|\Lambda\left(X^{\varepsilon}(t, x, w)\right)\right\|_{p, s}$.

Proof: For simplicity, we give a proof in the case of $d=1$. Let $p \in(1, \infty)$, $s \in \mathbb{R}$ and $t>0$ be arbitrary. It is enough to prove that $\left\|f\left(X\left(\varepsilon^{2} t, x, w\right)\right)\right\|_{p, s}=$ $\left\|f\left(X^{\varepsilon}(t, x, w)\right)\right\|_{p, s}$ for each $f \in \mathscr{S}(\mathbb{R})$. By the Veretennikov-Krylov formula (see Veretennikov and Krylov, 1976, p.279, Theorem 4), we have

$$
\begin{aligned}
& J_{n}\left[f\left(X^{\varepsilon}(t, x, w)\right)\right] \\
& =\int_{0}^{t} \cdots \int_{0}^{r_{2}}\left(P_{r_{1}}^{\varepsilon} Q_{r_{2}-r_{1}}^{\varepsilon} \cdots Q_{r_{n}-r_{n-1}}^{\varepsilon} Q_{t-r_{n}}^{\varepsilon} f\right)(x) \mathrm{d} w\left(r_{1}\right) \cdots \mathrm{d} w\left(r_{n}\right),
\end{aligned}
$$


where $\left(P_{r}^{\varepsilon} f\right)(z):=\mathbf{E}\left[f\left(X^{\varepsilon}(r, z, w)\right)\right]$ for $z \in \mathbb{R}, Q_{r}^{\varepsilon} f:=A^{\varepsilon}\left(P_{r}^{\varepsilon} f\right)$ and $A^{\varepsilon}:=\varepsilon \sigma \frac{\mathrm{d}}{\mathrm{d} x}$. In the case $\varepsilon=1$, we will write $P_{r}:=P_{r}^{1}, A:=A^{1}$ and $Q_{r}:=Q_{r}^{1}$ for simplicity. Therefore, we have to show that

$$
\begin{aligned}
& \left\{\int_{0}^{\varepsilon^{2} t} \cdots \int_{0}^{\varepsilon^{2} r_{2}}\left(P_{r_{1}} Q_{r_{2}-r_{1}} \cdots Q_{r_{k}-r_{k-1}} Q_{\varepsilon^{2} t-r_{k}} f\right)(x) \mathrm{d} w\left(r_{1}\right) \cdots \mathrm{d} w\left(r_{k}\right)\right\}_{k=0}^{n} \\
& =\left\{\int_{0}^{t} \cdots \int_{0}^{r_{2}}\left(P_{r_{1}}^{\varepsilon} Q_{r_{2}-r_{1}}^{\varepsilon} \cdots Q_{r_{k}-r_{k-1}}^{\varepsilon} Q_{t-r_{k}}^{\varepsilon} f\right)(x) \mathrm{d} w\left(r_{1}\right) \cdots \mathrm{d} w\left(r_{k}\right)\right\}_{k=0}^{n}
\end{aligned}
$$

in law for each $n \in \mathbb{N}$, and hence by the scaling property of Brownian motion: $\left(\varepsilon^{-1} w\left(\varepsilon^{2} t\right)\right)_{t \geqslant 0}=(w(t))_{t \geqslant 0}$ in law, it suffices to show that

$$
\begin{aligned}
& \left(P_{\varepsilon^{2} r_{1}} Q_{\varepsilon^{2} r_{2}-\varepsilon^{2} r_{1}} \cdots Q_{\varepsilon^{2} r_{n}-\varepsilon^{2} r_{n-1}} Q_{\varepsilon^{2} t-\varepsilon^{2} r_{n}} f\right)(x) \\
& =\varepsilon^{-n}\left(P_{r_{1}}^{\varepsilon} Q_{r_{2}-r_{1}}^{\varepsilon} \cdots Q_{r_{n}-r_{n-1}}^{\varepsilon} Q_{t-r_{n}}^{\varepsilon} f\right)(x)
\end{aligned}
$$

for each $0<r_{1}<\cdots<r_{n}<t$. But this is clear since we have $P_{\varepsilon^{2} r}=P_{r}^{\varepsilon}$, $A=\varepsilon^{-1} A^{\varepsilon}$ and $Q_{\varepsilon^{2} r}=\varepsilon^{-1} Q_{r}^{\varepsilon}$ for each $r>0$.

For each $\varepsilon>0$ and $x \in \mathbb{R}^{d}$, we set

$$
F(\varepsilon, x, w):=\frac{X^{\varepsilon}(1, x, w)-x}{\varepsilon} .
$$

Then the following two conditions are equivalent (see Watanabe, 1987, Theorem $3.4)$ :

$\circ$ (H2), i.e., there exists $\lambda>0$ such that

$$
\lambda|\xi|^{2} \leqslant\left\langle\xi,\left(\sigma \sigma^{*}\right)(x) \xi\right\rangle_{\mathbb{R}^{d}}, \quad \text { for all } \xi \in \mathbb{R}^{d} .
$$

$\circ$ the family $\{F(\varepsilon, x, w)\}_{\varepsilon>0}$ is uniformly non-degenerate.

Proposition 3.8. Let $d=1$ and $x \in \mathbb{R}$. Suppose (H1) and $\sigma(x)^{2}>0$. Then for any $p \in(1, \infty)$, we have $\sup _{0<\varepsilon \leqslant 1}\left\|\delta_{0}(F(\varepsilon, x, w))\right\|_{p,-2}<+\infty$.

Proof: Let $\phi(z):=\left(1+z^{2}-\triangle\right)^{-1} \delta_{0}(z) \in \mathscr{S}_{0}$ and take $q \in(1, \infty)$ so that $1 / p+1 / q=$ 1. Then for each $J \in \mathbb{D}^{\infty}$, we have

$$
\begin{aligned}
\mathbf{E}\left[\delta_{0}(F(\varepsilon, x, w)) J\right] & =\mathbf{E}\left[\left(\left(1+z^{2}-\triangle\right) \phi\right)(F(\varepsilon, x, w)) J\right] \\
& =\mathbf{E}\left[\phi(F(\varepsilon, x, w)) l_{\varepsilon}(J)\right]
\end{aligned}
$$

where $l_{\varepsilon}(J) \in \mathbb{D}^{\infty}$ is of the form

$$
l_{\varepsilon}(J)=\left\langle P_{0}(\varepsilon, w), D J\right\rangle_{H}+\left\langle P_{1}(\varepsilon, w), D^{2} J\right\rangle_{H \otimes H}
$$

for some $P_{i}(\varepsilon, w) \in \mathbb{D}^{\infty}\left(H^{\otimes i}\right), i=1,2$, both of which are polynomials in $F(\varepsilon, x, w)$, its derivatives up to the second order and $\left\|D X^{\varepsilon}(1, x, w)\right\|_{H}^{-2}$ (see e.g., Watanabe, 1987, equation (2.20)).

Take $q^{\prime} \in(1, q)$. Since $\{F(\varepsilon, x, w)\}_{\varepsilon>0}$ is uniformly non-degenerate, there exists $c_{0}>0$ such that

$$
\left\|l_{\varepsilon}(J)\right\|_{q^{\prime}} \leqslant c_{0}\|J\|_{q, 2} \quad \text { for all } \varepsilon \in(0,1] \text { and } J \in \mathbb{D}^{\infty} .
$$

Therefore we have for each $J \in \mathbb{D}^{\infty}$,

$$
\left|\mathbf{E}\left[\delta_{0}(F(\varepsilon, x, w)) J\right]\right| \leqslant\|\phi\|_{\infty}\left\|l_{\varepsilon}(J)\right\|_{q^{\prime}} \leqslant c_{0}\|\phi\|_{\infty}\|J\|_{q, 2}
$$

which implies $\sup _{0<\varepsilon \leqslant 1}\left\|\delta_{0}(F(\varepsilon, x, w))\right\|_{p,-2} \leqslant c_{0}\|\phi\|_{\infty}<+\infty$.

Second, we recall the next fact (see Ikeda and Watanabe, 1989, Chapter V, Section 8, p.384) to prove Theorem 3.4. 
Proposition 3.9. Let $x \in \mathbb{R}^{d}$ and suppose that (H1) and (H2). Then for any $p \in(1, \infty)$ and $k \in \mathbb{Z}_{\geqslant 0}$, there exists $C>0$ such that

$$
\|\phi \circ F(\varepsilon, x, w)\|_{p,-2 k} \leqslant C\|\phi\|_{-2 k} \quad \text { for } \phi \in \mathscr{S}\left(\mathbb{R}^{d}\right), \varepsilon \in(0, T] .
$$

The last tool we need is the following.

Lemma 3.10. Let $x \in \mathbb{R}^{d}$.

(i) If (H1) and (H2) hold, then there exist $K>0, T_{0} \in(0, T]$ with the following property: For each $k_{1}, \cdots, k_{d} \in \mathbb{Z}_{\geqslant 0}$ and $p \in(1, \infty)$, there exist constants $\nu_{0}, c_{1}, c_{2}>0$ such that

$$
\left\|\partial_{1}^{k_{1}} \cdots \partial_{d}^{k_{d}} \delta_{y}(X(t, x, w))\right\|_{p,-(n+2)} \leqslant c_{1} t^{-\nu_{0}} \exp \left\{-c_{2} \frac{|x-y|}{t}\right\}
$$

for each $t \in\left(0, T_{0}\right]$ and $y \in \mathbb{R}$ such that $|y| \geqslant K$.

(ii) If (H1), (H2) and (H4) hold, then for each $k_{1}, \cdots, k_{d} \in \mathbb{Z}_{\geqslant 0}, K>|x|$ and $p \in(1, \infty)$, there exist constants $\nu_{0}, c_{1}, c_{2}>0$ such that

$$
\left\|\partial_{1}^{k_{1}} \cdots \partial_{d}^{k_{d}} \delta_{y}(X(t, x, w))\right\|_{p,-(n+2)} \leqslant c_{1} t^{-\nu_{0}} \exp \left\{-c_{2} \frac{|x-y|^{2}}{t}\right\}
$$

for each $t \in(0, T]$ and $y \in \mathbb{R}$ such that $|y| \geqslant K$.

Here, $n:=k_{1}+\cdots+k_{d}$ in both cases.

Proof: (i) Assume (H1) and (H2). For simplicity of notation, we prove the case $d=1$. The case $d \geqslant 2$ can be proved by a similar argument. It suffices to prove that there exist $K>0$ and $T_{0} \in(0, T]$ with the following property:

For each $n \in \mathbb{Z}_{\geqslant 0}$ and $p \in(1, \infty)$, there exist constants $\nu_{0}, c_{1}, c_{2}>0$ such that

$$
\left|\mathbf{E}\left[J \delta_{y}^{(n)}\left(X_{t}\right)\right]\right| \leqslant c_{1} t^{-\nu_{0}} \exp \left\{-c_{2} \frac{|x-y|}{t}\right\}\|J\|_{p, n+2},
$$

for each $J \in \mathbb{D}^{\infty}, t \in\left(0, T_{0}\right]$ and $y \in \mathbb{R}$ with $|y| \geqslant K$.

Let $y \in \mathbb{R}$ be arbitrary and $\varphi(z):=\left(1+z^{2}-\triangle\right)^{-1} \delta_{y}(z) \in \mathscr{S}_{0}$. Take a $C^{\infty}$. function $\phi: \mathbb{R} \rightarrow \mathbb{R}$ such that

$$
\phi(\xi)= \begin{cases}1 & \text { if } \xi \leqslant 1 / 3 \\ 0 & \text { if } \xi \geqslant 2 / 3\end{cases}
$$

and then we set $\psi_{y}(z):=\phi\left(\frac{z-y}{\mid x-y}\right)$. Let $p \in(1, \infty)$ be arbitrary and let $q \in(1, \infty)$ be such that $1 / p+1 / q=1$. Further take $q^{\prime} \in(1, q)$. Since $\psi_{y} \delta_{y}=\delta_{y}$, we have for each $J \in \mathbb{D}^{\infty}$ that

$$
\begin{aligned}
\mathbf{E}\left[J \delta_{y}^{(n)}\left(X_{t}\right)\right] & =\mathbf{E}\left[J \psi_{y}\left(X_{t}\right)\left(\frac{\mathrm{d}^{n}}{\mathrm{~d} z^{n}}\left(1+z^{2}-\triangle\right) \varphi\right)\left(X_{t}\right)\right] \\
& =\mathbf{E}\left[\varphi\left(X_{t}\right)\left\{\sum_{j=0}^{n+2} \psi_{y}^{(j)}\left(X_{t}\right) l_{j, t}(J)\right\}\right]
\end{aligned}
$$

where $\psi_{y}^{(j)}$ is the $j$-th derivative of $\psi_{y}$ (with convention that $\psi_{y}^{(0)}=\psi_{y}$ ) and each $l_{j, t}(J)$ is of the form

$$
P_{0}(t, w) J+\left\langle P_{1}(t, w), D J\right\rangle_{H}+\cdots+\left\langle P_{n+2}(t, w), D^{n+2} J\right\rangle_{H^{\otimes(n+2)}}
$$

for some $P_{i}(t, w) \in \mathbb{D}^{\infty}\left(H^{\otimes i}\right), i=0,1, \cdots, n+2$, which is a polynomial in $X_{t}=$ $X(t, x, w)$, its derivatives and $\|D X(t, x, w)\|_{H}^{-2}$, but does not depend on $\psi_{y}$. 

to

Note that $\psi_{y}(z) \equiv 0$ for $|z-x|<\frac{|y-x|}{3}$, and hence the last term in (3.6) equals

$$
\mathbf{E}\left[\left(\sum_{j=0}^{n+2}\left(\varphi \psi_{y}^{(j)}\right)\left(X_{t}\right) l_{j, t}(J)\right) 1_{\left\{\left|X_{t}-x\right| \geqslant \frac{|y-x|}{3}\right\}}\right] .
$$

Therefore, by taking $p^{\prime} \in(1, \infty)$ such that $1 / p^{\prime}+1 / q^{\prime}=1$, we have

$$
\left|\mathbf{E}\left[J \delta_{y}^{(n)}\left(X_{t}\right)\right]\right| \leqslant \sum_{j=0}^{n+2}\left\|\varphi \psi_{y}^{(j)}\right\|_{\infty}\left\|l_{j, t}(J)\right\|_{q^{\prime}} \mathbf{P}\left(\left|X_{t}-x\right| \geqslant \frac{|y-x|}{3}\right)^{1 / p^{\prime}} .
$$

Henceforth we shall focus on each factor in the last equation.

First, since $\psi_{y}^{(j)}(z)=|x-y|^{-j} \phi^{(j)}((z-y) /|x-y|)$ for $j=0,1, \cdots, n+2$, and by Lemma 2.2 we have

$$
\sup _{\substack{y \in \mathbb{R} \\|y| \geqslant K}}\left\|\varphi \psi_{y}^{(j)}\right\|_{\infty}<+\infty \quad \text { for } K>0 \text { and } j=0,1, \cdots, n+2 .
$$

Second, it is well known that for each $r \in(1, \infty)$, there exist $\nu, c_{1}^{\prime}=c_{1}^{\prime}(r)>0$ such that

$$
\|\| D X_{t}\left\|_{H}^{-2}\right\|_{r} \leqslant c_{1}^{\prime} t^{-\nu} \quad \text { for any } t \in(0, T]
$$

(see Kusuoka and Stroock, 1985, (3.25) Corollary p.22 or Ikeda and Watanabe, 1989, Chapter V, Section 10, Theorem 10.2). Now, bearing in mind the form (3.7), we see that there exist $\nu_{0}, c_{1}^{\prime \prime}>0$ such that

$$
\left\|l_{j, t}(J)\right\|_{q^{\prime}} \leqslant c_{1}^{\prime \prime} t^{-\nu_{0}}\|J\|_{p, 2} \quad \text { for } j=0,1, \cdots, n+2 \text { and } t \in(0, T] .
$$

Third, we shall prove that there exist $K>0$ and $T_{0} \in(0, T]$ with the following property: there exist $c_{1}^{\prime \prime \prime}, c_{2}>0$ such that

$$
\mathbf{P}\left(\left|X_{t}-x\right| \geqslant \frac{|y-x|}{3}\right) \leqslant c_{1}^{\prime \prime \prime} \exp \left\{-c_{2} \frac{|x-y|}{t}\right\}
$$

for all $t \in\left(0, T_{0}\right]$ and $y \in \mathbb{R}$ with $|y| \geqslant K$. For this, we recall the following general fact (see Ikeda and Watanabe, 1989, Chapter V, section 10, Lemma 10.5):

Let $\kappa>0$ and $X=\left(X_{t}\right)_{0 \leqslant t \leqslant T}$ be a one-dimensional continuous semimartingale with its Doob-Mayer decomposition $X_{t}=X_{0}+M_{t}+$ $A_{t}$ such that $\langle M\rangle_{t}=\int_{0}^{t} \alpha(s) \mathrm{d} s, A_{t}=\int_{0}^{t} \beta(s) \mathrm{d} s$ and

$$
\sup _{0 \leqslant t \leqslant T} \max \{|\alpha(t)|,|\beta(t)|\} \leqslant \kappa \text {. }
$$

Then for any $a>0$ and $t \in\left(0, \min \left\{\frac{a}{2 \kappa}, T\right\}\right]$, it holds that

$$
\mathbf{P}\left(\tau_{a}<t\right) \leqslant \frac{4}{\sqrt{\pi a}} \exp \left(-\frac{a^{2}}{8 \kappa t}\right)
$$

where $\tau_{a}:=\inf \left\{t>0:\left|X_{t}-X_{0}\right|>a\right\}$.

Since we have assumed (H1), $\sigma^{2}$ and $b$ are Lipschitz continuous. Thus there exists $\alpha, \beta \geqslant 0$ such that $\max \left\{\left|\sigma^{2}(y)\right|,|b(y)|\right\} \leqslant \alpha|y-x|+\beta$ for any $y \in \mathbb{R}$. Put $\kappa(z):=\alpha z+\beta$ and fix $y \in \mathbb{R}$ arbitrarily. Let $T_{0} \in(0, T]$ so that $1-6 \alpha T_{0}>0$. Define $\tau:=: \tau_{|y-x| / 3}:=\inf \left\{t>0:\left|X_{t}-x\right|>\frac{|y-x|}{3}\right\}$. Then the stopped process $X^{\tau}=\left(X_{t \wedge \tau}\right)_{t \geqslant 0}$ is a continuous semi-martingale satisfying

$$
X_{t \wedge \tau}=x+\int_{0}^{t \wedge \tau} \sigma_{y}\left(X_{s \wedge \tau}\right) \mathrm{d} w(s)+\int_{0}^{t \wedge \tau} b_{y}\left(X_{s \wedge \tau}\right) \mathrm{d} s,
$$


where $\sigma_{y}(z):=\min \{\sigma(z), \kappa(|y-x|)\}$ and $b_{y}(z):=\min \{b(z), \kappa(|y-x|)\}$.

By setting $a=\frac{|y-x|}{3}$ in the above, we see that if $|y-x| \geqslant \xi_{0}:=\max \left\{\frac{6 \beta T_{0}}{1-6 \alpha T_{0}}, 1\right\}>$ 0 and $0<t \leqslant T_{0}$ (here, note that $\frac{\xi_{0}}{6\left(\alpha \xi_{0}+\beta\right)} \geqslant T_{0}$ since $\xi \mapsto \frac{\xi}{6(\alpha \xi+\beta)}$ is a nondecreasing function) then

$$
\begin{aligned}
\mathbf{P}\left(\left|X_{t}-X_{0}\right|>\frac{|y-x|}{3}\right) & \leqslant \mathbf{P}\left(\left|X_{t}^{\tau_{a}}-X_{0}\right|>\frac{|y-x|}{3}\right) \\
& \leqslant \mathbf{P}\left(\tau_{a}<t\right) \\
& \leqslant \frac{4}{\sqrt{\pi \frac{|y-x|}{3}}} \exp \left(-\frac{\left.\mid \frac{|y-x|}{3}\right)^{2}}{8(\alpha|y-x|+\beta) t}\right) \\
& \leqslant \frac{4 \sqrt{3}}{\sqrt{\pi \xi_{0}}} \exp \left(-\frac{|y-x|^{2}}{72(\alpha|y-x|+\beta|y-x|) t}\right) \\
& =\frac{4 \sqrt{3}}{\sqrt{\pi \xi_{0}}} \exp \left(-\frac{|y-x|}{72(\alpha+\beta) t}\right) .
\end{aligned}
$$

Thus (3.11) is satisfied if we set $K:=|x|+\xi_{0}, c_{1}^{\prime \prime \prime}:=\frac{4 \sqrt{3}}{\sqrt{\pi \xi_{0}}}$ and $c_{2}:=(72(\alpha+\beta))^{-1}$.

Now, combining (3.8), (3.9), (3.10) and (3.11), we obtain the result.

(ii) Assume (H1), (H2) and (H4). In this case, $\sigma$ and $b$ are bounded, and hence the following well-known estimate is available: There exist $c_{1}^{\prime \prime \prime}, c_{2}>0$ such that

$$
\mathbf{P}\left(\left|X_{t}-x\right| \geqslant \frac{|y-x|}{3}\right) \leqslant c_{1}^{\prime \prime \prime} \exp \left\{-c_{2} \frac{|x-y|^{2}}{t}\right\}
$$

for all $t \in(0, T]$ and $x, y \in \mathbb{R}$. By using this instead of (3.11), and combining with (3.8), (3.9) and (3.10), we obtain the result.

We are now in a position to prove Theorem 3.4.

Proof of Theorem 3.4: (i) Let $K>0$ and $T_{0} \in(0, T]$ be as in Lemma 3.10-(i). Since

$$
\frac{|x-y|^{2}}{y^{2}} \geqslant \frac{(|x|-|y|)^{2}}{y^{2}}=\frac{y^{2}-2|x y|+x^{2}}{y^{2}} \rightarrow 1 \quad \text { as }|y| \rightarrow+\infty,
$$

there exists $K^{\prime}>0$ such that

$$
|x-y| \geqslant \frac{|y|}{2}, \quad \text { for any }|y|>K^{\prime} .
$$

Let $K^{\prime \prime}:=\max \left\{K, K^{\prime}\right\}$. Let $k \in \mathbb{N}$ be such that $\Lambda \in \mathscr{S}_{-2 k}\left(\mathbb{R}^{d}\right)$. Let $p \in(1, \infty)$ be arbitrary. We have

$$
\int_{0}^{T}\left\|\Lambda\left(X_{t}\right)\right\|_{p,-2 k} \mathrm{~d} t=\int_{0}^{T_{0}}\left\|\Lambda\left(X_{t}\right)\right\|_{p,-2 k} \mathrm{~d} t+\int_{T_{0}}^{T}\left\|\Lambda\left(X_{t}\right)\right\|_{p,-2 k} \mathrm{~d} t,
$$

here, the last term is finite since $(0, T] \ni t \mapsto \Lambda\left(X_{t}\right) \in \mathbb{D}_{p}^{-2 k}$ is continuous. The other term is estimated as

$$
\int_{0}^{T_{0}}\left\|\Lambda\left(X_{t}\right)\right\|_{p,-2 k} \mathrm{~d} t \leqslant \int_{0}^{T_{0}} \int_{\mathbb{R}}\left\|\delta_{y}\left(X_{t}\right)\right\|_{p,-2} \mu(\mathrm{d} y) \mathrm{d} t \leqslant I_{1}+I_{2}
$$


where

$$
\begin{aligned}
I_{1} & :=\int_{0}^{T_{0}} \int_{|y|>K^{\prime \prime}}\left\|\delta_{y}\left(X_{t}\right)\right\|_{p,-2} \mu(\mathrm{d} y) \mathrm{d} t, \\
I_{2} & :=\int_{0}^{T_{0}} \int_{|y| \leqslant K^{\prime \prime}}\left\|\delta_{y}\left(X_{t}\right)\right\|_{p,-2} \mu(\mathrm{d} y) \mathrm{d} t .
\end{aligned}
$$

We shall look at the integral $I_{1}$. By Lemma 3.10-(i) and (3.13), there exist $\nu_{0}, c_{1}, c_{2}>0$ such that we have

$$
\left\|\delta_{y}\left(X_{t}\right)\right\|_{p,-2} \leqslant c_{1} t^{-\nu_{0}} \mathrm{e}^{-c_{2} \frac{|x-y|}{t}} \leqslant c_{1} t^{-\nu_{0}} \exp \left(-c_{2} \frac{|y|}{2 t}\right)
$$

for $|y|>K^{\prime \prime}$ and $t \in\left(0, T_{0}\right]$. To dominate the last quantity, we shall prove that for some $c_{3}>0$, it holds that

$$
t^{-\nu_{0}} \exp \left(-c_{2} \frac{|y|}{2 t}\right) \leqslant c_{3} \exp \left(-c_{2} \frac{|y|}{4 t}\right) \text { for } t \in\left(0, T_{0}\right] \text { and }|y|>K^{\prime \prime} .
$$

Indeed, we have

$$
\frac{t^{-\nu_{0}} \exp \left(-c_{2} \frac{|y|}{2 t}\right)}{\exp \left(-c_{2} \frac{|y|}{4 t}\right)}=t^{-\nu_{0}} \exp \left(-c_{2} \frac{|y|}{4 t}\right) \leqslant t^{-\nu_{0}} \exp \left(-c_{2} \frac{K^{\prime \prime}}{4 t}\right) \rightarrow 0
$$

as $t \downarrow 0$, which proves (3.15). Combining (3.14) and (3.15), we obtain

$$
\begin{aligned}
I_{1} & \leqslant c_{1} c_{3} \int_{0}^{T_{0}} \int_{|y|>K^{\prime \prime}} \exp \left\{-c_{2} \frac{|y|}{4 t}\right\} \mu(\mathrm{d} y) \mathrm{d} t \\
& \leqslant c_{1} c_{3} T \int_{|y|>K^{\prime \prime}} \exp \left\{-c_{2} \frac{|y|}{4 T}\right\} \mu(\mathrm{d} y)=c_{1} c_{3} T\langle\Lambda, f\rangle<+\infty
\end{aligned}
$$

where $f \in \mathscr{S}(\mathbb{R})$ is given by $f(y):=\phi(y) \mathrm{e}^{-c_{2}|y| /(4 T)}, y \in \mathbb{R}$ and $\phi$ is a $C^{\infty}$-function such that $\phi=0$ on a neighbourhood of 0 and $\phi(y)=1$ if $|y| \geqslant K^{\prime \prime}$.

Next we turn to $I_{2}$. By Proposition 3.9 and with noting that $\sup _{a \in \mathbb{R}}\left\|\delta_{a}\right\|_{-2}<$ $+\infty$ (Proposition 2.2), we have

$$
\begin{aligned}
& t^{1 / 2} \sup _{y \in \mathbb{R}}\left\|\delta_{y}(X(t, x, w))\right\|_{p,-2}=t^{1 / 2} \sup _{y \in \mathbb{R}}\left\|\delta_{y}\left(X^{\sqrt{t}}(1, x, w)\right)\right\|_{p,-2} \\
& =\sup _{y \in \mathbb{R}}\left\|\delta_{(y-x) / \sqrt{t}}(F(\sqrt{t}, x, w))\right\|_{p,-2} \leqslant C \sup _{a \in \mathbb{R}}\left\|\delta_{a}\right\|_{-2}
\end{aligned}
$$

for each $t>0$. Hence we can conclude that there exists $C^{\prime}>0$ such that

$$
\left\|\delta_{y}(X(t, x, w))\right\|_{p,-2} \leqslant C^{\prime} t^{-1 / 2} \text { for }|y| \leqslant K^{\prime \prime}, t \in(0, T] .
$$

Now, it is easy to deduce that

$$
I_{2} \leqslant C^{\prime} \mu\left(\left\{y \in \mathbb{R}:|y| \leqslant K^{\prime \prime}\right\}\right) \int_{0}^{T} t^{-1 / 2} \mathrm{~d} t<+\infty .
$$

(ii) is proved similarly by using Lemma 3.10-(ii) instead of Lemma 3.10-(i).

Recall that the support of $\Lambda \in \mathscr{D}^{\prime}\left(\mathbb{R}^{d}\right)$ is defined as the complement of

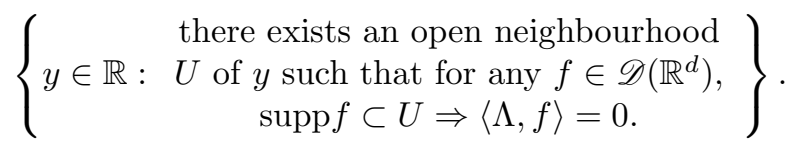

The proof of the following lemma will be given after Proposition 3.12 below. 
Lemma 3.11. Let $x \in \mathbb{R}^{d}$ and $\Lambda \in \mathscr{D}^{\prime}\left(\mathbb{R}^{d}\right)$ be such that $\operatorname{supp} \Lambda \not \supset x$. Then there exists $k \in \mathbb{Z}_{\geqslant 0}$ such that

$$
\lim _{t \downarrow 0}\|\Lambda(X(t, x, w))\|_{p,-k}=0 \quad \text { for each } p \in(1, \infty),
$$

if either one of the following holds:

(i) (H1), (H2) and $\Lambda \in \mathscr{S}^{\prime}\left(\mathbb{R}^{d}\right)$.

(ii) (H1), (H2), (H4) and $\Lambda \in \mathscr{E}^{\prime}\left(\mathbb{R}^{d}\right)$.

From this, the following is immediate.

Proposition 3.12. Let $x \in \mathbb{R}^{d}$ and $\Lambda \in \mathscr{D}^{\prime}\left(\mathbb{R}^{d}\right)$ be such that $\operatorname{supp} \Lambda \not \supset x$. Under the assumption in Lemma 3.11, there exists $k \in \mathbb{Z}_{\geqslant 0}$ such that

$$
\int_{0}^{T}\|\Lambda(X(t, x, w))\|_{p,-k}^{p} \mathrm{~d} t<+\infty \quad \text { for each } p \in(1, \infty) .
$$

Proof of Lemma 3.11: We shall prove under the assumption (ii). The proof in the case (i) is omitted since one can prove similarly.

We assume $d=1$ just for simplicity. Let $p \in(1, \infty)$ be arbitrary. By Proposition 2.4, we can write $\Lambda=\frac{\mathrm{d}^{k}}{\mathrm{~d} x^{k}}[\exp (k|x|) f(x)]$, where $k$ is a nonnegative integer and $f: \mathbb{R} \rightarrow \mathbb{R}$ is a bounded continuous function.

Since $x \notin \operatorname{supp} \Lambda$, we have $r_{0}:=\inf \{|x-y|: y \in \operatorname{supp} \Lambda\}>0$. Let $\Omega:=$ $\cup_{y \in \operatorname{supp} \Lambda} B_{r_{0} / 2}(y)$ (i.e., the $\left(r_{0} / 2\right)$-neighbourhood of $\operatorname{supp} \Lambda$ ), where $B_{r}(y)$ is the open ball with center $y$ and radius $r$. Then, we have $\Lambda \in \mathscr{D}^{\prime}(\Omega)$, and the function $f$ can be rearranged so that $\operatorname{supp} f \subset \Omega$.

Now let $\varepsilon>0$ and $J \in \mathbb{D}^{\infty}$ be arbitrary. Putting $\widetilde{e}_{k}(x)=e_{k}(|x|)=\exp (k|x|)$, we have

$$
\begin{aligned}
& \mathbf{E}\left[\Lambda\left(X^{\varepsilon}(1, x, w)\right) J\right]=\mathbf{E}\left[\left(\widetilde{e}_{k} f\right)^{(k)}\left(X^{\varepsilon}(1, x, w)\right) J\right] \\
& =\mathbf{E}\left[\exp \left(k\left|X^{\varepsilon}(1, x, w)\right|\right) f\left(X^{\varepsilon}(1, x, w)\right) l_{\varepsilon}(J)\right]
\end{aligned}
$$

where $l_{\varepsilon}(J) \in \mathbb{D}^{\infty}$ is of the form

$$
l_{\varepsilon}(J)=\sum_{j=0}^{k}\left\langle P_{j}(\varepsilon, w), D^{j} J\right\rangle_{H^{\otimes j}}
$$

for some $P_{j}(\varepsilon, w) \in \mathbb{D}^{\infty}\left(H^{\otimes j}\right), j=0,1, \cdots, k$, which are polynomials in $F(\varepsilon, x, w)$, its derivatives up to the order $k$, and $\left|D X^{\varepsilon}(t, x, w)\right|_{H}^{-2}$. Take $q^{\prime} \in(1, q)$. Since $\{F(\varepsilon, x, w)\}_{\varepsilon>0}$ is uniformly non-degenerate, there exists $c_{0}, \nu>0$ such that

$$
\left\|l_{\varepsilon}(J)\right\|_{q^{\prime}} \leqslant c_{0} \varepsilon^{-\nu}\|J\|_{q, k} \quad \text { for all } \varepsilon \in(0, T] \text { and } J \in \mathbb{D}^{\infty} .
$$

Therefore by taking $p^{\prime} \in(1, \infty)$ such that $1 / p^{\prime}+1 / q^{\prime}=1$, we have

$$
\begin{aligned}
& \left|\mathbf{E}\left[\Lambda\left(X^{\varepsilon}(1, x, w)\right) J\right]\right| \\
& \leqslant\left\|\exp \left(k\left|X^{\varepsilon}(1, x, w)\right|\right) f\left(X^{\varepsilon}(1, x, w)\right)\right\|_{p^{\prime}}\left\|l_{\varepsilon}(J)\right\|_{q^{\prime}} \\
& \leqslant c_{0} \varepsilon^{-\nu}\left\|\exp \left(k\left|X^{\varepsilon}(1, x, w)\right|\right) f\left(X^{\varepsilon}(1, x, w)\right)\right\|_{p^{\prime}}\|J\|_{q, k},
\end{aligned}
$$


which implies $\left\|\Lambda\left(X^{\varepsilon}(1, x, w)\right)\right\|_{p,-k} \leqslant c_{0} \varepsilon^{-\nu}\left\|\exp \left(k\left|X^{\varepsilon}(1, x, w)\right|\right) f\left(X^{\varepsilon}(1, x, w)\right)\right\|_{p^{\prime}}$ for any $\varepsilon>0$. By Proposition 3.7, we obtain

$$
\begin{aligned}
& \|\Lambda(X(t, x, w))\|_{p,-k}=\left\|\Lambda\left(X^{\sqrt{t}}(1, x, w)\right)\right\|_{p,-k} \\
& \leqslant c_{0} t^{-\nu / 2}\left\|\exp \left(k\left|X^{\sqrt{t}}(1, x, w)\right|\right) f\left(X^{\sqrt{t}}(1, x, w)\right)\right\|_{p^{\prime}} \\
& =c_{0} t^{-\nu / 2}\left\|\exp \left(k\left|X_{t}\right|\right) f\left(X_{t}\right) 1_{\left\{\left|X_{t}-x\right|>\frac{r_{0}}{2}\right\}}\right\|_{p^{\prime}} .
\end{aligned}
$$

By Lemma 3.10-(ii), we find that for any $r>0, \mathbf{E}[\exp (r|X(t, x, w)|)]=O\left(t^{-\nu^{\prime}}\right)$ as $t \downarrow 0$ for some $\nu^{\prime}>0$. However we have $\mathbf{P}\left(\left|X_{t}-x\right|>\frac{r_{0}}{2}\right)=O\left(\mathrm{e}^{-r_{0}^{2} /\left(c_{1} t\right)}\right)$ as $t \downarrow 0$ for some constant $c_{1}>0$, so that the last quantity converges to zero as $t \downarrow 0$, and hence get the conclusion.

3.3. Hölder continuity of local time in space variable: a special case. In this section, we assume $d=1,(\mathrm{H} 1)$ and (H3). Let $X=\left(X_{t}\right)_{t \geqslant 0}$ be a unique strong solution to the following one-dimensional stochastic differential equation

$$
\mathrm{d} X_{t}=\sigma\left(X_{t}\right) \mathrm{d} w(t)+\frac{1}{2} \sigma\left(X_{t}\right) \sigma^{\prime}\left(X_{t}\right) \mathrm{d} t, \quad X_{0}=x \in \mathbb{R},
$$

or equivalently,

$$
\mathrm{d} X_{t}=\sigma\left(X_{t}\right) \circ \mathrm{d} w(t), \quad X_{0}=x .
$$

The main purpose in this section is to prove Theorem 1.2.

Note that the object $\sigma(y)^{2} \int_{0}^{t} \delta_{y}\left(X_{u}\right) \mathrm{d} u$ in Theorem 1.2 is identified with the symmetric local time associated to the diffusion process $\left(X_{t}\right)_{t \geqslant 0}$. See Remark 4.15.

The Hermite polynomials $H_{n}, n \in \mathbb{Z}_{\geqslant 0}$ are defined by $H_{0}(x)=1$ and $H_{n}(x):=$ $\partial^{* n} 1(x)$ for $n \in \mathbb{N}$ and $x \in \mathbb{R}$, where the operator $\partial^{*}$ is given by

$$
\partial^{*} f(x):=-f^{\prime}(x)+x f(x), \quad x \in \mathbb{R}
$$

for any differentiable function $f: \mathbb{R} \rightarrow \mathbb{R}$.

The proof of Theorem 1.2 starts from this paragraph. Let $y, z \in \mathbb{R}$ be arbitrary. Let $A=A_{z}:=\sigma(z) \frac{\mathrm{d}}{\mathrm{d} z}$ and $p_{t}\left(z_{1}, z_{2}\right)$ be the transition-density function of $X$. For each $t \geqslant 0$, the Krylov-Veretennikov formula tells us that $\delta_{a}\left(X_{t}\right)=\sum_{n=0}^{\infty} J_{n}\left[\delta_{a}\left(X_{t}\right)\right]$ (the convergence is in $\mathbb{D}_{2}^{-\infty}$ ) for every $a \in \mathbb{R}$, where

$$
J_{n}\left[\delta_{a}\left(X_{t}\right)\right]=\int_{0 \leqslant t_{1}<\cdots<t_{n} \leqslant t} \Pi_{n}(x ; t, a)\left[t_{1}, \cdots, t_{n}\right] \mathrm{d} w\left(t_{1}\right) \cdots \mathrm{d} w\left(t_{n}\right)
$$

and

$$
\begin{aligned}
& \Pi_{n}(x ; t, a)\left[t_{1}, \cdots, t_{n}\right] \\
& =\int_{\mathbb{R}} \cdots \int_{\mathbb{R}} p_{t_{1}}\left(x, z_{1}\right) A_{z_{1}}\left[p_{t_{2}-t_{1}}\left(z_{1}, z_{2}\right)\right] \cdots A_{z_{n}}\left[p_{t-t_{n}}\left(z_{n}, a\right)\right] \mathrm{d} z_{1} \cdots \mathrm{d} z_{n} .
\end{aligned}
$$

We remark that the stochastic integral in (3.17) is well defined since the squareintegrability of each component $\left(t_{1}, \cdots, t_{n}\right) \mapsto \Pi_{n}(x ; t, a)\left[t_{1}, \cdots, t_{n}\right]$ of the chaos kernel $\left\{\Pi_{n}(x ; t, a)\right\}_{n=0}^{\infty}$ is established in Watanabe (1994b, Corollary 4.5), in a more general situation. Since the generator $L=\frac{1}{2} A^{2}$ commutes with $A$, we have

$$
\begin{aligned}
& \Pi_{n}(x ; t, a)\left[t_{1}, \cdots, t_{n}\right]=\left(\mathrm{e}^{t_{1} L} A \mathrm{e}^{\left(t_{2}-t_{1}\right) L} \cdots A \mathrm{e}^{\left(t_{n}-t_{n-1}\right) L} A \mathrm{e}^{\left(t-t_{n}\right) L}\right) \delta_{a} \\
& =\left[A^{n} \exp \left(\left\{t_{1}+\left(t_{2}-t_{1}\right)+\cdots+\left(t_{n}-t_{n-1}\right)+\left(t-t_{n}\right)\right\} L\right)\right] \delta_{a}=\left(A^{n} \mathrm{e}^{t L}\right)\left(\delta_{a}\right)
\end{aligned}
$$


in the distributional sense. By using $\left(\mathrm{e}^{t L} \delta_{a}\right)(x)=p_{t}(x, a)$, the formula (3.17) reduces to

$$
J_{n}\left[\delta_{a}\left(X_{t}\right)\right]=\left[A_{x}^{n} p_{t}(x, a)\right] \int_{0 \leqslant t_{1}<\cdots<t_{n} \leqslant t} \mathrm{~d} w\left(t_{1}\right) \cdots \mathrm{d} w\left(t_{n}\right) .
$$

Therefore, by using Corollary 3.6, we have

$$
\sigma(a) \int_{0}^{1} \delta_{a}\left(X_{s}\right) \mathrm{d} s=\sum_{n=0}^{\infty} \int_{0}^{1}\left[\sigma(a) A_{x}^{n} p_{t}(x, a)\right] \int_{0 \leqslant t_{1}<\cdots<t_{n} \leqslant t} \mathrm{~d} w\left(t_{1}\right) \cdots \mathrm{d} w\left(t_{n}\right) \mathrm{d} t .
$$

Hence we have

$$
\begin{aligned}
& \left\|\sigma(y) \int_{0}^{1} \delta_{y}\left(X_{t}\right) \mathrm{d} t-\sigma(z) \int_{0}^{1} \delta_{z}\left(X_{t}\right) \mathrm{d} t\right\|_{2, s}^{2} \\
& =\sum_{n=0}^{\infty}(1+n)^{s} \mathbf{E}\left[\left\{\int_{0}^{1}\left[\sigma(y) A_{x}^{n} p_{t}(x, y)-\sigma(z) A_{x}^{n} p_{t}(x, z)\right]\right.\right. \\
& \left.\left.\quad \times \int_{0 \leqslant t_{1}<\cdots<t_{n} \leqslant t} \mathrm{~d} w\left(t_{1}\right) \cdots \mathrm{d} w\left(t_{n}\right) \mathrm{d} t\right\}^{2}\right],
\end{aligned}
$$

and so we need to compute

$$
\begin{aligned}
& I_{n}:=\mathbf{E}\left[\left\{\int_{0}^{1}\left[\sigma(y) A_{x}^{n} p_{t}(x, y)-\sigma(z) A_{x}^{n} p_{t}(x, z)\right] \int_{0 \leqslant t_{1}<\cdots<t_{n} \leqslant t} \mathrm{~d} w\left(t_{1}\right) \cdots \mathrm{d} w\left(t_{n}\right) \mathrm{d} t\right\}^{2}\right] \\
& =2 \int_{0 \leqslant s<t \leqslant 1} \mathrm{~d} s \mathrm{~d} t\left\{\sigma(y) A_{x}^{n} p_{s}(x, y)-\sigma(z) A_{x}^{n} p_{s}(x, z)\right\}\left\{\sigma(y) A_{x}^{n} p_{t}(x, y)-\sigma(z) A_{x}^{n} p_{t}(x, z)\right\} \\
& \quad \times \mathbf{E}\left[\int_{0 \leqslant t_{1}<\cdots<t_{n} \leqslant s} \mathrm{~d} w\left(t_{1}\right) \cdots \mathrm{d} w\left(t_{n}\right) \int_{0 \leqslant t_{1}<\cdots<t_{n} \leqslant t} \mathrm{~d} w\left(t_{1}\right) \cdots \mathrm{d} w\left(t_{n}\right)\right] \\
& =\frac{2}{n !} \int_{0 \leqslant s<t \leqslant 1} s^{n} \mathrm{~d} s \mathrm{~d} t\left\{\sigma(y) A_{x}^{n} p_{s}(x, y)-\sigma(z) A_{x}^{n} p_{s}(x, z)\right\}\left\{\sigma(y) A_{x}^{n} p_{t}(x, y)-\sigma(z) A_{x}^{n} p_{t}(x, z)\right\} .
\end{aligned}
$$

Since $\sigma$ is Lipschitz continuous (which is because of (H1)), the vector field $A=\sigma(z)(\mathrm{d} / \mathrm{d} z)$ is complete, so that one can associate the one-parameter group of diffeomorphisms $\left\{\mathrm{e}^{s A}\right\}_{s \in \mathbb{R}}$. Note that $\mathrm{e}^{s A}(x)$ for each $x \in \mathbb{R}$ is defined by $\frac{\mathrm{d}}{\mathrm{d} s} \mathrm{e}^{s A}(x)=\sigma\left(\mathrm{e}^{s A}(x)\right)$ and $\left.\mathrm{e}^{s A}(x)\right|_{s=0}=x$.

Lemma 3.13. $\frac{\mathrm{d}}{\mathrm{d} u} \mathrm{e}^{u A}(x)=\sigma(x) \frac{\partial}{\partial x} \mathrm{e}^{u A}(x)$ for every $u \in \mathbb{R}$ and $x \in \mathbb{R}$.

Proof: By the homomorphism property: $\mathrm{e}^{(s+u) A}=\mathrm{e}^{s A} \circ \mathrm{e}^{u A}$, we have $\frac{\mathrm{d}}{\mathrm{d} s} \mathrm{e}^{s A}(x)=$ $\left.\frac{\mathrm{d}}{\mathrm{d} u}\right|_{u=0} \mathrm{e}^{(s+u) A}(x)=\left.\frac{\mathrm{d}}{\mathrm{d} u}\right|_{u=0} \mathrm{e}^{s A}\left(\mathrm{e}^{u A}(x)\right)=\sigma(x) \frac{\partial}{\partial x} \mathrm{e}^{s A}(x)$.

To continue the calculation of $I_{n}$, we shall look at $A_{x} p_{t}(x, a)$. The unique strong solution $X=\left(X_{t}\right)_{t \geqslant 0}$ is now expressed by $X_{t}=\mathrm{e}^{w(t) A}(x)$ (To check this, just apply 
the Itô formula for $\left.\mathrm{e}^{w(t) A}(x)\right)$. Therefore by using Lemma 3.13, we have

$$
\begin{aligned}
& A_{x} p_{t}(x, a)=A_{x} \mathbf{E}\left[\delta_{a}\left(\mathrm{e}^{w(t) A}(x)\right)\right] \\
& =\sigma(x) \frac{\partial}{\partial x} \int_{\mathbb{R}} \delta_{a}\left(\mathrm{e}^{\sqrt{t} u A}(x)\right) \frac{\mathrm{e}^{-u^{2} / 2}}{\sqrt{2 \pi}} \mathrm{d} u \\
& =\int_{\mathbb{R}}\left(\sigma(x) \frac{\partial}{\partial x} \mathrm{e}^{\sqrt{t} u A}(x)\right) \delta_{a}^{\prime}\left(\mathrm{e}^{\sqrt{t} u A}(x)\right) \frac{\mathrm{e}^{-u^{2} / 2}}{\sqrt{2 \pi}} \mathrm{d} u \\
& =\int_{\mathbb{R}}\left(\frac{1}{\sqrt{t}} \frac{\mathrm{d}}{\mathrm{d} u} \mathrm{e}^{\sqrt{t} u A}(x)\right) \delta_{a}^{\prime}\left(\mathrm{e}^{\sqrt{t} u A}(x)\right) \frac{\mathrm{e}^{-u^{2} / 2}}{\sqrt{2 \pi}} \mathrm{d} u \\
& =\frac{1}{\sqrt{t}} \int_{\mathbb{R}}\left(\frac{\mathrm{d}}{\mathrm{d} u} \delta_{a}\left(\mathrm{e}^{\sqrt{t} u A}(x)\right)\right) \frac{\mathrm{e}^{-u^{2} / 2}}{\sqrt{2 \pi}} \mathrm{d} u \\
& =\int_{\mathbb{R}} \delta_{a}\left(\mathrm{e}^{\sqrt{t} u A}(x)\right)\left(\frac{-1}{\sqrt{t}} \frac{\mathrm{d}}{\mathrm{d} u} \frac{\mathrm{e}^{-u^{2} / 2}}{\sqrt{2 \pi}}\right) \mathrm{d} u,
\end{aligned}
$$

where the integral is understood as the coupling of the Schwartz distribution and the test function. By the repetition of the above procedure, we obtain

$$
\begin{aligned}
A_{x}^{n} p_{t}(x, a) & =\int_{\mathbb{R}} \delta_{a}\left(\mathrm{e}^{\sqrt{t} u A}(x)\right)\left\{\frac{(-1)^{n}}{t^{n / 2}} \frac{\mathrm{d}^{n}}{\mathrm{~d} u^{n}} \frac{\mathrm{e}^{-u^{2} / 2}}{\sqrt{2 \pi}}\right\} \mathrm{d} u \\
& =\int_{\mathbb{R}} \delta_{a}\left(\mathrm{e}^{\sqrt{t} u A}(x)\right) \frac{1}{t^{n / 2}} H_{n}(u) \frac{\mathrm{e}^{-u^{2} / 2}}{\sqrt{2 \pi}} \mathrm{d} u
\end{aligned}
$$

For each $t>0$, define a mapping $\varphi: \mathbb{R} \rightarrow \mathbb{R}$ by $\varphi(u):=\mathrm{e}^{\sqrt{t} u A}(x)$. Since $\varphi$ is continuously differentiable and $\left|\varphi^{\prime}(u)\right|=\sqrt{t}|\sigma(\varphi(u))| \geqslant \sqrt{t} \lambda^{1 / 2}$ for every $u$, where $\lambda>0$ is the constant appeared in (H3), we see that $\varphi$ is a diffeomorphism. Hence we can apply the change of variables $b=\varphi(u)^{1}$ (and then $\left.\mathrm{d} u / \mathrm{d} b=(\sqrt{t} \sigma(b))^{-1}\right)$ in the above integral to get

$$
\begin{aligned}
A_{x}^{n} p_{t}(x, a) & =\int_{\mathbb{R}} \delta_{a}(b) \frac{1}{t^{(n+1) / 2}} H_{n}\left(\varphi^{-1}(b)\right) \frac{\mathrm{e}^{-\left(\varphi^{-1}(b)\right)^{2} / 2}}{\sqrt{2 \pi} \sigma(b)} \mathrm{d} b \\
& =\frac{1}{t^{(n+1) / 2}} H_{n}\left(\varphi^{-1}(a)\right) \frac{\mathrm{e}^{-\left(\varphi^{-1}(a)\right)^{2} / 2}}{\sqrt{2 \pi} \sigma(a)} .
\end{aligned}
$$

By using Lemma A.1-(i), we obtain the formula

$$
A_{x}^{n} p_{t}(x, a)=\frac{(-1)^{n} t^{-(n+1) / 2}}{2 \pi \sigma(a)} \int_{-\infty}^{\infty}(i \xi)^{n} \mathrm{e}^{-\xi^{2} / 2} \mathrm{e}^{i \xi \varphi^{-1}(a)} \mathrm{d} \xi
$$

Therefore by using (3.19),

$$
\begin{aligned}
& \sigma(y) A_{x}^{n} p_{t}(x, y)-\sigma(z) A_{x}^{n} p_{t}(x, z) \\
& =\frac{(-1)^{n} t^{-(n+1) / 2}}{2 \pi} \int_{-\infty}^{\infty}(i \xi)^{n} \mathrm{e}^{-\xi^{2} / 2}\left\{\mathrm{e}^{i \xi \varphi^{-1}(y)}-\mathrm{e}^{i \xi \varphi^{-1}(z)}\right\} \mathrm{d} \xi \\
& =: \mathbb{I}_{n} .
\end{aligned}
$$

Lemma 3.14. For any $\alpha \in[0,1]$ and $\theta \in \mathbb{R}$, we have $\left|\mathrm{e}^{i \theta}-1\right| \leqslant 2|\theta|^{\alpha}$ with the convention $0^{0}:=1$.

\footnotetext{
$1_{\text {when }} t=1$, we see that $\varphi^{-1}(z)=\int_{x}^{z} \frac{\mathrm{d} a}{\sigma(a)}$ and the stochastic process $\varphi^{-1}\left(X_{s}\right)$, which is nothing but the Wiener process $w(s)$, is known as the Lamperti transformation of $X$.
} 
Proof: Let $\alpha \in[0,1]$. If $\theta \in \mathbb{R} \backslash(-1,1)$, then we see that $\left|\mathrm{e}^{i \theta}-1\right| \leqslant 2 \leqslant 2|\theta|^{\alpha}$. On the other hand, for $\theta \in(-1,1),\left|\mathrm{e}^{i \theta}-1\right| \leqslant|\theta| \leqslant|\theta|^{\alpha} \leqslant 2|\theta|^{\alpha}$ because $\alpha \in[0,1]$. Thus we obtain $\left|\mathrm{e}^{i \theta}-1\right| \leqslant 2|\theta|^{\alpha}$ for all $\theta \in \mathbb{R}$.

Let $\beta \in[0,1)$ be arbitrary. By Lemma 3.14, we have

$$
\begin{aligned}
\left|I_{n}\right| & \leqslant \frac{t^{-(n+1) / 2}}{\pi}\left|\varphi^{-1}(y)-\varphi^{-1}(z)\right|^{\beta} \int_{-\infty}^{\infty}|\xi|^{n+\beta} \mathrm{e}^{-\xi^{2} / 2} \mathrm{~d} \xi \\
& =\frac{t^{-(n+1) / 2} 2^{(n+\beta-1) / 2}}{\pi} \Gamma\left(\frac{n+\beta+1}{2}\right)\left|\varphi^{-1}(y)-\varphi^{-1}(z)\right|^{\beta} .
\end{aligned}
$$

Since $\frac{\mathrm{d}}{\mathrm{d} b} \varphi^{-1}(b)=\frac{1}{\sqrt{t} \sigma(b)}$, we have $\left|\varphi^{-1}(y)-\varphi^{-1}(z)\right|=t^{-1 / 2}\left|\int_{y}^{z} \sigma(b)^{-1} \mathrm{~d} b\right|$, so that

$$
\left|I_{n}\right| \leqslant \frac{t^{-(n+\beta+1) / 2} 2^{(n+\beta-1) / 2}}{\pi \lambda^{\beta / 2}} \Gamma\left(\frac{n+\beta+1}{2}\right)|y-z|^{\beta},
$$

where it is recalled again that $\lambda>0$ is what appeared in (H3).

Substituting (3.21) into (3.20),

$$
\begin{aligned}
& \left|\sigma(y) A_{x}^{n} p_{t}(x, y)-\sigma(z) A_{x}^{n} p_{t}(x, z)\right| \\
& \leqslant c_{2} t^{-(n+\beta+1) / 2} 2^{(n+\beta-1) / 2} \Gamma\left(\frac{n+\beta+1}{2}\right)|y-z|^{\beta}
\end{aligned}
$$

where $c_{2}:=\left(\pi \lambda^{\beta / 2}\right)^{-1}$. Note that $c_{2}$ does not depend on $y$ and $z$. With putting

$$
c_{3}(n):=\Gamma\left(\frac{n+\beta+1}{2}\right),
$$

we have obtained

$$
\left|\sigma(y) A_{x}^{n} p_{t}(x, y)-\sigma(z) A_{x}^{n} p_{t}(x, z)\right| \leqslant c_{2} t^{-(n+\beta+1) / 2} 2^{(n+\beta-1) / 2} c_{3}(n)|y-z|^{\beta} .
$$

Now, by substituting (3.22) into (3.18), we have

$$
I_{n} \leqslant \frac{\left(c_{2}\right)^{2}}{n !} 2^{n+\beta} c_{3}(n)^{2}|y-z|^{2 \beta} \int_{0}^{1} s^{n} s^{-(n+\beta+1) / 2} \mathrm{~d} s \int_{s}^{1} t^{-(n+\beta+1) / 2} \mathrm{~d} t .
$$

Note that the last iterated integral is finite because $\beta<1$, and gives

$$
\int_{0}^{1} s^{n} s^{-(n+\beta+1) / 2} \mathrm{~d} s \int_{s}^{1} t^{-(n+\beta+1) / 2} \mathrm{~d} t=\frac{2}{(1-\beta)(n-\beta+1)} .
$$

Hence we have

$$
I_{n} \leqslant \frac{\left(c_{2}\right)^{2}}{(1-\beta)}|y-z|^{2 \beta} \frac{2^{n+\beta+1} c_{3}(n)^{2}}{n !(n-\beta+1)} .
$$

Finally we have

$$
\begin{aligned}
& \left\|\sigma(y) \int_{0}^{1} \delta_{y}\left(X_{t}\right) \mathrm{d} t-\sigma(z) \int_{0}^{1} \delta_{z}\left(X_{t}\right) \mathrm{d} t\right\|_{2, s}^{2}=\sum_{n=0}^{\infty}(1+n)^{s} I_{n} \\
& \leqslant \frac{\left(c_{2}\right)^{2}}{(1-\beta)}|y-z|^{2 \beta} \sum_{n=0}^{\infty}(1+n)^{s} \frac{2^{n+\beta+1} c_{3}(n)^{2}}{n !(n-\beta+1)}
\end{aligned}
$$

By Stirling's formula, we see that the quantity

$$
(1+n)^{s} \frac{2^{n+\beta+1} c_{3}(n)^{2}}{n !(n-\beta+1)}=(1+n)^{s} \frac{2^{n+\beta+1}}{n !(n-\beta+1)} \Gamma\left(\frac{n+\beta+1}{2}\right)^{2}
$$


behaves like

$$
\begin{aligned}
& (1+n)^{s} \frac{2^{n+\beta+1}}{(n-\beta+1) \sqrt{2 \pi n}\left(\frac{n}{\mathrm{e}}\right)^{n}} \times\left\{\sqrt{\pi(n+\beta-1)}\left(\frac{n+\beta-1}{2 \mathrm{e}}\right)^{\frac{n+\beta-1}{2}}\right\}^{2} \\
& =\frac{(1+n)^{s} 2^{n+\beta+1}}{(n-\beta+1) \sqrt{2 \pi n}\left(\frac{n}{\mathrm{e}}\right)^{n}} \pi(n+\beta-1)\left(\frac{n+\beta-1}{2 \mathrm{e}}\right)^{n+\beta-1}=O\left(n^{s+\beta-\frac{3}{2}}\right)
\end{aligned}
$$

as $n \rightarrow \infty$ for each $\beta$. Hence the sum converges if $s+\beta-\frac{3}{2}<-1$, i.e., $s+\beta<\frac{1}{2}$. The proof of Theorem 1.2 finishes.

Proof of Corollary 1.3: This is clear from Theorem 1.2 and the inequality

$$
\begin{aligned}
& \left|\sigma(y) \int_{0}^{1} p_{t}(x, y) \mathrm{d} t-\sigma(z) \int_{0}^{1} p_{t}(x, z) \mathrm{d} t\right| \\
& =\left|\mathbf{E}\left[\sigma(y) \int_{0}^{1} \delta_{y}\left(X_{t}\right) \mathrm{d} t-\sigma(z) \int_{0}^{1} \delta_{z}\left(X_{t}\right) \mathrm{d} t\right]\right| \\
& \leqslant\left\|\sigma(y) \int_{0}^{1} \delta_{y}\left(X_{t}\right) \mathrm{d} t-\sigma(z) \int_{0}^{1} \delta_{z}\left(X_{t}\right) \mathrm{d} t\right\|_{2,-1 / 2} .
\end{aligned}
$$

\section{Itô's Formula for Generalized Wiener Functionals}

Let $w=(w(t))_{t \geqslant 0}$ be the $d$-dimensional Wiener process with $w(0)=0$ and let $\left(\mathcal{F}_{t}^{w}\right)_{t \geqslant 0}$ be the filtration generated by $w: \mathcal{F}_{t}^{w}:=\sigma(w(s): 0 \leqslant s \leqslant t)$, for $t \geqslant 0$. Similarly to the previous section, we fix $x \in \mathbb{R}^{d}$ and consider the following $d$-dimensional stochastic differential equation

$$
\mathrm{d} X_{t}=\sigma\left(X_{t}\right) \mathrm{d} w(t)+b\left(X_{t}\right) \mathrm{d} t, \quad X_{0}=x \in \mathbb{R}^{d} .
$$

Also in this section, we assume the conditions (H1) and (H2). We denote by $\{X(t, x, w)\}_{t \geqslant 0}$ a unique strong solution $X=\left(X_{t}\right)_{t \geqslant 0}$ to (4.1).

4.1. Stochastic integrals of pull-backs by diffusion. For each $J \in \mathbb{D}^{\infty}$, we will denote by $(D J)^{i}$ the $i$-th component of $D J \in \mathbb{D}^{\infty}(H): D J=\left((D J)^{1}, \cdots,(D J)^{d}\right)$ (Recall $H$ is the Cameron-Martin subspace of the $d$-dimensional Wiener space). For each $t \in[0, T]$, the evaluation map evt $: H \ni h \mapsto h(t) \in \mathbb{R}^{d}$ naturally induces a map id $\otimes \mathrm{ev}_{t}: L_{2}(H) \cong L_{2} \otimes H \rightarrow L_{2} \otimes \mathbb{R}^{d}$ and then we write $D_{t} J:=:\left(\left(D_{t} J\right)^{1}, \cdots,\left(D_{t} J\right)^{d}\right):=\frac{\mathrm{d}}{\mathrm{d} t}\left(\mathrm{id} \otimes \mathrm{ev}_{t}\right)(D J)$ for a.a. $t \in[0, T]$.

Let $\Lambda \in \mathscr{D}^{\prime}\left(\mathbb{R}^{d}\right)$. Throughout this section, we assume either one of

$\circ(\mathrm{H} 1),(\mathrm{H} 2)$ and $\Lambda \in \mathscr{S}^{\prime}\left(\mathbb{R}^{d}\right)$;

$\circ(\mathrm{H} 1),(\mathrm{H} 2),(\mathrm{H} 4)$ and $\Lambda \in \mathscr{E}^{\prime}\left(\mathbb{R}^{d}\right)$.

Then $\Lambda(X(t, x, w))$ is defined as a generalized Wiener functional. If $\int_{0}^{T}\|\Lambda(X(t, x, w))\|_{2,-k}^{2} \mathrm{~d} t$ is finite for some $k \in \mathbb{N}$, we define the stochastic integrals $\int_{0}^{T} \Lambda(X(t, x, w)) \mathrm{d} w^{i}(t), i=1, \cdots, d$ as elements in $\mathbb{D}^{-\infty}$ via the pairing

$$
\mathbf{E}\left[\left(\int_{0}^{T} \Lambda(X(t, x, w)) \mathrm{d} w^{i}(t)\right) J\right]=\int_{0}^{T} \mathbf{E}\left[\Lambda(X(t, x, w))\left(D_{t} J\right)^{i}\right] \mathrm{d} t,
$$

for $J \in \mathbb{D}^{\infty}$ and $i=1,2, \cdots, d$. We define the stochastic integral $\int_{s}^{T} \Lambda(X(t, x, w)) \mathrm{d} w^{i}(t)$ similarly for each $0<s \leqslant T$. 
This pairing is well defined because of the following:

Proposition 4.1. For each $k \in \mathbb{N}$ and $i=1,2, \cdots, d$, there exists a constant $C>0$ such that

$$
\int_{0}^{T}\left|\mathbf{E}\left[\Lambda(X(t, x, w))\left(D_{t} J\right)^{i}\right]\right| \mathrm{d} t \leqslant C\left\{\int_{0}^{T}\|\Lambda(X(t, x, w))\|_{2,-k}^{2} \mathrm{~d} t\right\}^{1 / 2}\|J\|_{2, k+1}
$$

for all $J \in \mathbb{D}^{\infty}$.

Proof: For simplicity of notation, we prove in the case $d=1$. For each $k \in \mathbb{N}$ and $J \in \mathbb{D}^{\infty}$,

$$
\begin{aligned}
& \int_{0}^{T}\left|\mathbf{E}\left[\Lambda\left(X_{t}\right) D_{t} J\right]\right| \mathrm{d} t \leqslant \int_{0}^{T}\left\|\Lambda\left(X_{t}\right)\right\|_{2,-k}\left\|D_{t} J\right\|_{2, k} \mathrm{~d} t \\
& \leqslant\left\{\int_{0}^{T}\left\|\Lambda\left(X_{t}\right)\right\|_{2,-k}^{2} \mathrm{~d} t\right\}^{1 / 2}\left\{\int_{0}^{T}\left\|D_{t} J\right\|_{2, k}^{2} \mathrm{~d} t\right\}^{1 / 2} .
\end{aligned}
$$

By Meyer's inequality, there exist constants $c^{\prime}, C^{\prime}>0$ such that

$$
c^{\prime}\left\|D^{k^{\prime}} F\right\|_{2} \leqslant\|F\|_{2, k^{\prime}} \leqslant C^{\prime} \sum_{l=0}^{k^{\prime}}\left\|D^{l} F\right\|_{2}, \quad \text { for all } F \in \mathbb{D}_{2}^{k^{\prime}}
$$

for $k^{\prime}=1,2, \cdots, k+1$. Therefore we have

$$
\begin{aligned}
& \left\|D_{t} J\right\|_{2, k}^{2} \leqslant\left(C^{\prime}\right)^{2}\left\{\sum_{l=0}^{k}\left\|D^{l} D_{t} J\right\|_{2}\right\}^{2} \leqslant\left(C^{\prime}\right)^{2}(k+1) \sum_{l=0}^{k}\left\|D^{l} D_{t} J\right\|_{2}^{2} \\
& =\left(C^{\prime}\right)^{2}(k+1) \sum_{l=0}^{k} \mathbf{E}\left[\left\|D^{l} D_{t} J\right\|_{H^{\otimes l}}^{2}\right] \\
& =C^{\prime \prime}\left\{\mathbf{E}\left[\left(D_{t} J\right)^{2}\right]+\sum_{l=1}^{k} \int_{0}^{T} \cdots \int_{0}^{T} \mathbf{E}\left[\left(D_{s_{l}} \cdots D_{s_{1}} D_{t} J\right)^{2}\right] \mathrm{d} s_{1} \cdots \mathrm{d} s_{l}\right\},
\end{aligned}
$$

where $C^{\prime \prime}:=\left(C^{\prime}\right)^{2}(k+1)$, so that

$$
\begin{aligned}
& \int_{0}^{T}\left\|D_{t} J\right\|_{2, k}^{2} \mathrm{~d} t \\
& \leqslant C^{\prime \prime} \sum_{l=1}^{k+1} \int_{0}^{T} \cdots \int_{0}^{T} \mathbf{E}\left[\left(D_{u_{l}} \cdots D_{u_{1}} J\right)^{2}\right] \mathrm{d} u_{1} \cdots \mathrm{d} u_{l} \\
& \leqslant C^{\prime \prime} \sum_{l=0}^{k+1}\left\|D^{l} J\right\|_{2}^{2} \leqslant\left(c^{\prime}\right)^{-2} C^{\prime \prime}\|J\|_{2, k+1}^{2} .
\end{aligned}
$$

Hence by substituting this into (4.3), we get the result.

Remark 4.2. (a) As is easily seen, the stochastic integral $\int_{0}^{T} \Lambda\left(X_{t}\right) \mathrm{d} w^{i}(t)$ has another expression:

$$
\int_{0}^{T} \Lambda\left(X_{t}\right) \mathrm{d} w^{i}(t)=D^{*}\left[(0, \cdots, \underbrace{\int_{0}^{\bullet} \Lambda\left(X_{u}\right) \mathrm{d} u}_{i \text {-th position }}, \cdots, 0)\right] .
$$

Hence it coincides with the Skorokhod integral as long as the object standing on the right of $D^{*}$ lies in the domain $\mathbb{D}_{2}^{1}(H)$ and then automatically coincides with the Itô 
integral because of the adaptedness. In fact, in view of the Clark-Ocone formula, every $J \in L_{2}$ can be written as $J=\mathbf{E}[J]+\sum_{i=1}^{d} \int_{0}^{T} \mathbf{E}\left[\left(D_{t} J\right)^{i} \mid \mathcal{F}_{t}^{w}\right] \mathrm{d} w^{i}(t)$ and then (4.2) is just the Itô isometry. Therefore, our stochastic integral may be a natural extension of the classical anticipative stochastic integral to this distributional setting.

(b) By Proposition 4.1, we have $\int_{0}^{T} \Lambda\left(X_{t}\right) \mathrm{d} w^{i}(t) \in \mathbb{D}_{2}^{-(k+1)}$ for $i=1,2, \cdots, d$ provided $\int_{0}^{T}\left\|\Lambda\left(X_{t}\right)\right\|_{2,-k}^{2} \mathrm{~d} t<+\infty$.

The following is the main result in this section. This is a version of a result by Uemura (2004, Proposition 1).

Theorem 4.3. Let $s \in \mathbb{R}, p \geqslant 2$ and assume that $(0, T] \ni t \mapsto \Lambda(X(t, x, w)) \in \mathbb{D}_{p}^{s}$ is continuous. Then we have

$$
\int_{0}^{T} \Lambda(X(t, x, w)) \mathrm{d} w^{i}(t) \in \mathbb{D}_{p}^{s}, \quad \text { for } i=1,2, \cdots, d
$$

provided either one of the following

(i) $\lim _{t \downarrow 0}\|\Lambda(X(t, x, w))\|_{p, s}=0$.

(ii) $s \geqslant 0$ and $\int_{0}^{T}\|\Lambda(X(t, x, w))\|_{p, s}^{2} \mathrm{~d} t<\infty$.

Remark 4.4. (a) See also a remark just after Lemma 4.12 for verification of the continuity assumption.

(b) From the proof of Theorem 4.3, we would find that

$$
\int_{t_{0}}^{T} \Lambda\left(X_{t}\right) \mathrm{d} w^{i}(t) \in \mathbb{D}_{p}^{s} \quad \text { for any } t_{0}>0
$$

and $i=1,2, \cdots, d$ if $(0, T] \ni t \mapsto \Lambda\left(X_{t}\right) \in \mathbb{D}_{p}^{s}$ is continuous.

The proof of Theorem 4.3 mainly consists of the following series of Propositions 4.6, 4.7 and 4.8. We will give the proof at the last of this section.

Before the next definition, we note that $\mathbf{E}\left[F \mid \mathcal{F}_{t}^{w}\right] \in \mathbb{D}^{\infty}$ for every $t \geqslant 0$ if $F \in \mathbb{D}^{\infty}$.

Definition 4.5. Let $t \geqslant 0$. We say that a generalized Wiener functional $F \in \mathbb{D}^{-\infty}$ is $\mathcal{F}_{t}^{w}$-measurable if it holds that $\mathbf{E}[F G]=\mathbf{E}\left[F \mathbf{E}\left[G \mid \mathcal{F}_{t}^{w}\right]\right]$ for any $G \in \mathbb{D}^{\infty}$.

Proposition 4.6. Let $s \in \mathbb{R}$ and $p \geqslant 2$. Then there exists $c=c(p, s, T)>0$ such that, for any mapping $F:(0, T] \ni t \mapsto F(t) \in \mathbb{D}_{p}^{s}$ with $F(t)$ is $\mathcal{F}_{t}^{w}$-measurable for any $t \in(0, T]$, any division $0=t_{0}<t_{1}<\cdots<t_{n}=T$, and any $i=1,2, \cdots, d$, we have

$$
\left\|\sum_{k=2}^{n} F\left(t_{k-1}\right)\left(w^{i}\left(t_{k}\right)-w^{i}\left(t_{k-1}\right)\right)\right\|_{p, s}^{p} \leqslant c \sum_{k=2}^{n}\left\|F\left(t_{k-1}\right)\right\|_{p, s}^{p}\left(t_{k}-t_{k-1}\right) .
$$

Proof: Let $0=t_{0}<t_{1}<\cdots<t_{n}=T$ be any division of $[0, T]$ and set

$$
\Phi:=\sum_{k=2}^{n} F\left(t_{k-1}\right)\left(w^{i}\left(t_{k}\right)-w^{i}\left(t_{k-1}\right)\right) .
$$

To calculate $\|\Phi\|_{p, s}$, we begin with the chaos expansion of each $F\left(t_{k-1}\right)\left(w^{i}\left(t_{k}\right)-\right.$ $\left.w^{i}\left(t_{k-1}\right)\right)$. Noting $\mathbf{E}\left[F\left(t_{k-1}\right)\left(w^{i}\left(t_{k}\right)-w^{i}\left(t_{k-1}\right)\right)\right]=0$ and

$$
J_{m}\left[F\left(t_{k-1}\right)\left(w^{i}\left(t_{k}\right)-w^{i}\left(t_{k-1}\right)\right)\right]=J_{m-1}\left[F\left(t_{k-1}\right)\right]\left(w^{i}\left(t_{k}\right)-w^{i}\left(t_{k-1}\right)\right)
$$


for $m \geqslant 1$ (here we have used the condition that $F\left(t_{k-1}\right)$ is $\mathcal{F}_{t_{k-1}}^{w}$-measurable), one finds that the chaos expansion is given by

$$
F\left(t_{k-1}\right)\left(w^{i}\left(t_{k}\right)-w^{i}\left(t_{k-1}\right)\right)=\sum_{m=1}^{\infty} J_{m-1}\left[F\left(t_{k-1}\right)\right]\left(w^{i}\left(t_{k}\right)-w^{i}\left(t_{k-1}\right)\right),
$$

where $F\left(t_{k-1}\right)=\sum_{m=0}^{\infty} J_{m}\left[F\left(t_{k-1}\right)\right]$ is the chaos expansion of $F\left(t_{k-1}\right)$. Hence, by using (4.4), we have

$$
\begin{aligned}
& (I-\mathcal{L})^{s / 2} \sum_{k=2}^{n} F\left(t_{k-1}\right)\left(w^{i}\left(t_{k}\right)-w^{i}\left(t_{k-1}\right)\right) \\
& =\sum_{k=2}^{n} \sum_{m=1}^{\infty}(1+m)^{s / 2} J_{m-1}\left[F\left(t_{k-1}\right)\right]\left(w^{i}\left(t_{k}\right)-w^{i}\left(t_{k-1}\right)\right) \\
& =\sum_{m=0}^{\infty} \frac{(2+m)^{s / 2}}{(1+m)^{s / 2}} \sum_{k=2}^{n}(1+m)^{s / 2} J_{m}\left[F\left(t_{k-1}\right)\right]\left(w^{i}\left(t_{k}\right)-w^{i}\left(t_{k-1}\right)\right) .
\end{aligned}
$$

By Meyer's $L_{p}$-multiplier theorem (see e.g. Ikeda and Watanabe, 1989, Chapter V, Section 8, Lemma 8.2), there exists $c^{\prime}=c^{\prime}(p, s)>0$ such that

$$
\begin{aligned}
& \left\|\sum_{k=2}^{n} F\left(t_{k-1}\right)\left(w^{i}\left(t_{k}\right)-w^{i}\left(t_{k-1}\right)\right)\right\|_{p, s} \\
& =\left\|(I-\mathcal{L})^{s / 2} \sum_{k=2}^{n} F\left(t_{k-1}\right)\left(w^{i}\left(t_{k}\right)-w^{i}\left(t_{k-1}\right)\right)\right\|_{p} \\
& \leqslant c^{\prime}\left\|\sum_{m=0}^{\infty} \sum_{k=2}^{n}(1+m)^{s / 2} J_{m}\left[F\left(t_{k-1}\right)\right]\left(w^{i}\left(t_{k}\right)-w^{i}\left(t_{k-1}\right)\right)\right\|_{p} \\
& =c^{\prime}\left\|\sum_{k=2}^{n}\left[(I-\mathcal{L})^{s / 2} F\left(t_{k-1}\right)\right]\left(w^{i}\left(t_{k}\right)-w^{i}\left(t_{k-1}\right)\right)\right\|_{p} .
\end{aligned}
$$

Note that $(I-\mathcal{L})^{s / 2} F\left(t_{k-1}\right) \in L_{2}$ and is $\mathcal{F}_{t_{k-1}}^{w}$-measurable. Hence the last quantity in the $L_{p}$-norm can be written as

$$
\int_{0}^{T} \sum_{k=2}^{n}\left[(I-\mathcal{L})^{s / 2} F\left(t_{k-1}\right)\right] 1_{\left(t_{k-1}, t_{k}\right]}(t) \mathrm{d} w^{i}(t) .
$$

Thus by using the Burkholder-Davis-Gundy inequality, we get

$$
\begin{aligned}
& \left\|\sum_{k=2}^{n} F\left(t_{k-1}\right)\left(w^{i}\left(t_{k}\right)-w^{i}\left(t_{k-1}\right)\right)\right\|_{p, s}^{p} \\
& \leqslant c^{\prime \prime} \mathbf{E}\left[\left\{\int_{0}^{T} \sum_{k=2}^{n}\left[(I-\mathcal{L})^{s / 2} F\left(t_{k-1}\right)\right]^{2} 1_{\left(t_{k-1}, t_{k}\right]}(t) \mathrm{d} t\right\}^{p / 2}\right]
\end{aligned}
$$

for some constant $c^{\prime \prime}>0$. Finally, using the assumption $p \geqslant 2$ and the Jensen inequality, we reached

$$
\left\|\sum_{k=2}^{n} F\left(t_{k-1}\right)\left(w^{i}\left(t_{k}\right)-w^{i}\left(t_{k-1}\right)\right)\right\|_{p, s}^{p} \leqslant c \sum_{k=2}^{n}\left\|F\left(t_{k-1}\right)\right\|_{p, s}^{p}\left(t_{k}-t_{k-1}\right)
$$

for some constant $c>0$. 
Given $n \in \mathbb{N}, i=1,2, \cdots, d$ and the dyadic division $\left\{t_{k}:=k T / 2^{n}\right\}_{k=0}^{2^{n}}$ of $[0, T]$, we define

$$
\Phi_{n}:=\sum_{k=2}^{2^{n}} \Lambda\left(X\left(t_{k-1}, x, w\right)\right)\left(w^{i}\left(t_{k}\right)-w^{i}\left(t_{k-1}\right)\right) .
$$

Proposition 4.7. Let $s \in \mathbb{R}, p \geqslant 2$ and suppose that

(i) $(0, T] \ni t \mapsto \Lambda(X(t, x, w)) \in \mathbb{D}_{p}^{s}$ is continuous and

(ii) $\lim _{t \downarrow 0}\|\Lambda(X(t, x, w))\|_{p, s}=0$.

Then we have $\left\|\Phi_{n}-\Phi_{m}\right\|_{p, s} \rightarrow 0$ as $n, m \rightarrow \infty$.

Proof: Suppose that $n<m$ and let $t_{k}:=k T / 2^{n}$ and $u_{l}:=l T / 2^{m}$. Then we have

$$
\Phi_{n}-\Phi_{m}=\sum_{k=2}^{2^{n}} \sum_{\substack{l \in\left\{0,1, \cdots, 2^{m}\right\}: \\ t_{k-1}<u_{l} \leqslant t_{k}}}\left[\Lambda\left(X_{t_{k-1}}\right)-\Lambda\left(X_{u_{l}}\right)\right]\left(w^{i}\left(u_{l}\right)-w^{i}\left(u_{l-1}\right)\right)
$$

and hence by Proposition 4.6, we obtain

$$
\left\|\Phi_{n}-\Phi_{m}\right\|_{p, s}^{p} \leqslant c \sum_{k=2}^{2^{n}} \sum_{\substack{l \in\left\{0,1, \cdots, 2^{m}\right\}: \\ t_{k-1}<u_{l} \leqslant t_{k}}}\left\|\Lambda\left(X_{t_{k-1}}\right)-\Lambda\left(X_{u_{l}}\right)\right\|_{p, s}^{p}\left(u_{l}-u_{l-1}\right),
$$

for some constant $c>0$. By the assumption, the mapping $(0, T] \ni t \mapsto \Lambda\left(X_{t}\right) \in \mathbb{D}_{p}^{s}$ is uniformly continuous, from which, we easily get $\left\|\Phi_{n}-\Phi_{m}\right\|_{p, s} \rightarrow 0$ as $n \rightarrow \infty$.

Proposition 4.8. Suppose $k \in \mathbb{Z}_{\geqslant 0}$ and

(i) $(0, T] \ni t \mapsto \Lambda(X(t, x, w)) \in \mathbb{D}_{2}^{-k}$ is continuous;

(ii) $\lim _{t \downarrow 0}\|\Lambda(X(t, x, w))\|_{2,-k}=0$.

Then we have

$$
\left\|\Phi_{n}-\int_{0}^{T} \Lambda(X(t, x, w)) \mathrm{d} w^{i}(t)\right\|_{2,-(k+1)} \rightarrow 0 \quad \text { as } n \rightarrow \infty .
$$

Proof: The same argument in Proposition 4.1 leads us to

$$
\begin{aligned}
& \left\|\Phi_{n}-\int_{0}^{T} \Lambda\left(X_{t}\right) \mathrm{d} w^{i}(t)\right\|_{2,-(k+1)} \\
& \leqslant\left\{\int_{0}^{t_{1}}\left\|\Lambda\left(X_{t}\right)\right\|_{2,-k}^{2} \mathrm{~d} t+\sum_{k=2}^{2^{n}} \int_{t_{k-1}}^{t_{k}}\left\|\Lambda\left(X_{t_{k-1}}\right)-\Lambda\left(X_{t}\right)\right\|_{2,-k}^{2} \mathrm{~d} t\right\}^{1 / 2} .
\end{aligned}
$$

By the assumption, we have $(0, T] \ni t \mapsto \Lambda\left(X_{t}\right) \in \mathbb{D}_{2}^{-k}$ is uniformly continuous, and hence the above quantity converges to zero as $n \rightarrow \infty$.

Proof of Theorem 4.3: (i) Suppose that $\lim _{t \downarrow 0}\left\|\Lambda\left(X_{t}\right)\right\|_{p, s}=0$. In the first, suppose that $s<0$. Let $k$ be the largest integer, not exceeding $s$, i.e., $k=\max \left\{k^{\prime} \in \mathbb{Z}\right.$ : $\left.k^{\prime} \leqslant s\right\}$. By the assumption, the stochastic integral $\int_{0}^{T} \Lambda\left(X_{t}\right) \mathrm{d} w^{i}(t)$ is defined as an element in $\mathbb{D}_{2}^{k-1}$ (see Remark 4.2-(b)). On the other hand, Proposition 4.7 tells us that $\left\{\Phi_{n}\right\}_{n=1}^{\infty}$ is a Cauchy sequence in $\mathbb{D}_{p}^{s}$ and hence converges to some $\Phi \in \mathbb{D}_{p}^{s}$. Hence $\Phi_{n}$ converges to $\Phi$ also in $\mathbb{D}_{2}^{k-1}$. Now by Proposition 4.8 , it must be $\int_{0}^{T} \Lambda\left(X_{t}\right) \mathrm{d} w^{i}(t)=\lim _{n \rightarrow \infty} \Phi_{n}=\Phi$. Thus we have $\int_{0}^{T} \Lambda\left(X_{t}\right) \mathrm{d} w^{i}(t) \in \mathbb{D}_{p}^{s}$.

Second, suppose that $s \geqslant 0$. In this case, it is clearly satisfied that $\int_{0}^{T}\left\|\Lambda\left(X_{t}\right)\right\|_{p, s}^{p} \mathrm{~d} t<+\infty$, and hence it reduces to the case (ii). 
(ii) Suppose that $s \geqslant 0$ and $\int_{0}^{T}\left\|\Lambda\left(X_{t}\right)\right\|_{p, s}^{p} \mathrm{~d} t<+\infty$. Then by Proposition 4.6, we have

$$
\left\|\Phi_{n}\right\|_{p, s}^{p} \leqslant \text { const. } \sum_{k=2}^{2^{n}}\left\|\Lambda\left(X_{t_{k-1}}\right)\right\|_{p, s}^{p}\left(t_{k}-t_{k-1}\right) \rightarrow \int_{0}^{T}\left\|\Lambda\left(X_{t}\right)\right\|_{p, s}^{p} \mathrm{~d} t,
$$

as $n \rightarrow \infty$. Here, $t_{k}=k T / 2^{n}, k=0,1, \cdots, 2^{n}$. Hence $\left\{\Phi_{n}\right\}_{n=1}^{\infty}$ forms a bounded family in $\mathbb{D}_{p}^{s}$, so that by Alaoglu's theorem (for dual spaces of separable normed spaces), there exists a subsequence $\left\{\Phi_{n_{l}}\right\}_{l=1}^{\infty}$ and $\Phi \in \mathbb{D}_{p}^{s}$ such that $\Phi_{n_{l}} \rightarrow \Phi$ weakly in $\mathbb{D}_{p}^{s}$. In particular, since $s \geqslant 0$ and $p \geqslant 2$, this convergence is still valid in the weak topology on $L_{2}$. On the other hand, it is clearly satisfied that $\int_{0}^{T}\left\|\Lambda\left(X_{t}\right)\right\|_{2}^{2} \mathrm{~d} t<\infty$, and hence $\left\{\Lambda\left(X_{t}\right)\right\}_{t \geqslant 0}$ is now a square-integrable $\left(\mathcal{F}_{t}\right)_{t \geqslant 0}$-adapted process. The classical stochastic analysis proves that $\Phi_{n} \rightarrow \int_{0}^{T} \Lambda\left(X_{t}\right) \mathrm{d} w^{i}(t)$ in $L_{2}$. Therefore, it must be $\int_{0}^{T} \Lambda\left(X_{t}\right) \mathrm{d} w^{i}(t)=\lim _{l \rightarrow \infty} \Phi_{n_{l}}=\Phi$, and thus $\int_{0}^{T} \Lambda\left(X_{t}\right) \mathrm{d} w^{i}(t) \in \mathbb{D}_{p}^{s}$.

4.2. Distributional Itô's formula. Let $A_{i}, i=1,2, \cdots, d$ and $L$ be the vector fields and the second-order differential operator given by

$$
\begin{aligned}
& \left(A_{i} f\right)(z):=\sum_{k=1}^{d} \sigma_{i}^{k}(z) \frac{\partial f}{\partial z_{k}}(z) \\
& (L f)(z):=\frac{1}{2} \sum_{i, j=1}^{d}\left(\sigma \sigma^{*}\right)_{i}^{j}(z) \frac{\partial^{2} f}{\partial z_{i} \partial z_{j}}(z)+\sum_{i=1}^{d} b^{i}(z) \frac{\partial f}{\partial z_{i}}(z)
\end{aligned}
$$

for $f \in \mathscr{S}\left(\mathbb{R}^{d}\right)$ and $z \in \mathbb{R}^{d}$. In the case of $d=1$, the vector field $A_{1}$ will be denoted by $A$. Under the assumption (H1), these operators naturally act on $\mathscr{S}^{\prime}\left(\mathbb{R}^{d}\right)$ and $\mathscr{E}^{\prime}\left(\mathbb{R}^{d}\right)$.

Theorem 4.9 (cf. Kubo, 1983). Let $x \in \mathbb{R}^{d}$ and assume (H1) and (H2). Then for each $\Lambda \in \mathscr{S}^{\prime}\left(\mathbb{R}^{d}\right)$ and $t_{0} \in(0, T]$, we have

$$
\begin{aligned}
& \Lambda(X(T, x, w))-\Lambda\left(X\left(t_{0}, x, w\right)\right) \\
& =\sum_{i=1}^{d} \int_{t_{0}}^{T}\left(A_{i} \Lambda\right)(X(t, x, w)) \mathrm{d} w^{i}(t)+\int_{t_{0}}^{T}(L \Lambda)(X(t, x, w)) \mathrm{d} t \quad \text { in } \mathbb{D}^{-\infty} .
\end{aligned}
$$

Similarly, we have (4.5) for $\Lambda \in \mathscr{E}^{\prime}\left(\mathbb{R}^{d}\right)$ if we further assume (H4).

Proof: For simplicity of notation, we assume $d=1$. The case $d \geqslant 2$ is similar. Fix $t_{0}>0$. Suppose that $\Lambda \in \mathscr{S}_{-2 k}$. Then there exist $\phi_{n} \in \mathscr{S}(\mathbb{R}), n \in \mathbb{N}$ such that $\Lambda=\lim _{n \rightarrow \infty} \phi_{n}$ in $\mathscr{S}_{-2 k}$. By Itô's formula, we clearly have

$$
\phi_{n}\left(X_{T}\right)-\phi_{n}\left(X_{t_{0}}\right)=\int_{t_{0}}^{T}\left(A \phi_{n}\right)\left(X_{t}\right) \mathrm{d} w(t)+\int_{t_{0}}^{T}\left(L \phi_{n}\right)\left(X_{t}\right) \mathrm{d} t
$$

for each $n \in \mathbb{N}$. What we have to prove is the following: As $n \rightarrow \infty$,

(a) $\left\|\Lambda\left(X_{t}\right)-\phi_{n}\left(X_{t}\right)\right\|_{2,-2 k} \rightarrow 0$ for $t=t_{0}$ and $T$,

(b) $\left\|\int_{t_{0}}^{T}\left[A \Lambda\left(X_{t}\right)-A \phi_{n}\left(X_{t}\right)\right] \mathrm{d} w(t)\right\|_{2,-(2 k+2)} \rightarrow 0$,

(c) $\left\|\int_{t_{0}}^{T}\left[L \Lambda\left(X_{t}\right)-L \phi_{n}\left(X_{t}\right)\right] \mathrm{d} t\right\|_{2,-(2 k+2)} \rightarrow 0$. 
It is easy to show (a). In fact, for any $p \in(1,+\infty)$, we have the inequality

$$
\left\|\Lambda\left(X_{t}\right)-\phi_{n}\left(X_{t}\right)\right\|_{p,-2 k} \leqslant c_{0} t^{-K}\left|\Lambda-\phi_{n}\right|_{-2 k}
$$

where the constants $c_{0}>0$ and $K>0$ can depend on $p$ but not on $t$ and $\phi_{n}$ 's (see Ikeda and Watanabe, 1989, Chapter V, Section 9, Theorem 9.1 and Section 10, Theorem 10.2). We shall prove (b). By Proposition 4.1 and (4.2), we have

$$
\left\|\int_{t_{0}}^{T}\left[A \Lambda\left(X_{t}\right)-A \phi_{n}\left(X_{t}\right)\right] \mathrm{d} w(t)\right\|_{2,-(2 k+2)}^{2} \leqslant C \int_{t_{0}}^{T}\left\|\left[A\left(\Lambda-\phi_{n}\right)\right]\left(X_{t}\right)\right\|_{2,-(2 k+1)}^{2} \mathrm{~d} t .
$$

Next we shall show that there exists $c, \nu>0$ such that

$$
\left\|\left[A\left(\Lambda-\phi_{n}\right)\right]\left(X_{t}\right)\right\|_{2,-(2 k+1)}^{2} \leqslant c t^{-\nu}\left\|\left(\Lambda-\phi_{n}\right)\left(X_{t}\right)\right\|_{4,-2 k}^{2}
$$

for every $t \in\left[t_{0}, T\right]$, and then the above quantities converge to zero uniformly in $t \in\left[t_{0}, T\right]$ as $n \rightarrow \infty$, and hence (b) is proved. To prove (4.7), it suffices to show that: there exist $c, \nu>0$ such that

$$
\left|\mathbf{E}\left[\left(\sigma \Psi^{\prime}\right)\left(X_{t}\right) J\right]\right| \leqslant c t^{-\nu}\left\|\Psi\left(X_{t}\right)\right\|_{4,-2 k}\|J\|_{2,2 k+1}
$$

for each $\Psi \in \mathscr{S}_{-2 k}$ and $J \in \mathbb{D}^{\infty}$. In fact, we have

$$
\mathbf{E}\left[\left(\sigma \Psi^{\prime}\right)\left(X_{t}\right) J\right]=\mathbf{E}\left[\Psi\left(X_{t}\right)\left\{P_{0}(t) \sigma\left(X_{t}\right) J+\left\langle P_{1}(t), D\left(\sigma\left(X_{t}\right) J\right)\right\rangle_{H}\right\}\right]
$$

for some $P_{i}(t) \in \mathbb{D}^{\infty}\left(H^{\otimes i}\right), i=0,1$ which are polynomials in $X_{t}=X(t, x, w)$, its derivatives and $\left\|D X_{t}\right\|_{H}^{-2}$. Hence

$$
\begin{aligned}
& \left|\mathbf{E}\left[\left(\sigma \Psi^{\prime}\right)\left(X_{t}\right) J\right]\right| \\
& \leqslant\left\|\Psi\left(X_{t}\right)\right\|_{4,-2 k}\left\{\left\|P_{0}(t) \sigma\left(X_{t}\right) J\right\|_{4 / 3,2 k}+\left\|\left\langle P_{1}(t), D\left(\sigma\left(X_{t}\right) J\right)\right\rangle_{H}\right\|_{4 / 3,2 k}\right\} .
\end{aligned}
$$

Noting that $\frac{1}{2}+\frac{1}{4}=\frac{3}{4}<1$, we can make estimates

$$
\begin{aligned}
& \left\|P_{0}(t) \sigma\left(X_{t}\right) J\right\|_{4 / 3,2 k} \leqslant c^{\prime} t^{-\nu}\|J\|_{2,2 k}, \\
& \left\|\left\langle P_{1}(t), D\left(\sigma\left(X_{t}\right) J\right)\right\rangle_{H}\right\|_{4 / 3,2 k} \leqslant c^{\prime} t^{-\nu}\|J\|_{2,2 k+1}
\end{aligned}
$$

for each $t>0$, and for some constants $c^{\prime}, \nu>0$ (where, $c^{\prime}$ may depend on the derivatives of $\sigma$ up to the $(2 k+1)$-th order, which are assumed to be bounded by (H1)). Now (4.8) follows.

(c) is proved similarly. The statement for $\Lambda \in \mathscr{E}^{\prime}(\mathbb{R})$ is also proved similarly.

Theorem 4.10. Let $x \in \mathbb{R}^{d}$. Suppose (H1), (H2) and (H4). Let $f: \mathbb{R}^{d} \rightarrow \mathbb{R}$ be a locally-integrable function such that

(i) $f$ is continuous at $x$,

(ii) $f \in \mathscr{E}^{\prime}\left(\mathbb{R}^{d}\right)$,

(iii) $\int_{0}^{T}\left\|\left(A_{i} f\right)(X(t, x, w))\right\|_{2,-k}^{2} \mathrm{~d} t<+\infty$ for $i=1,2, \cdots, d$,

(iv) $\int_{0}^{T}\|(L f)(X(t, x, w))\|_{2,-k} \mathrm{~d} t<+\infty$

for some $k \in \mathbb{N}$. Then we have

$$
\begin{aligned}
& f(X(T, x, w))-f(x) \\
& =\sum_{i=1}^{d} \int_{0}^{T}\left(A_{i} f\right)(X(t, x, w)) \mathrm{d} w^{i}(t)+\int_{0}^{T}(L f)(X(t, x, w)) \mathrm{d} t \quad \text { in } \mathbb{D}^{-\infty} .
\end{aligned}
$$

The equation (4.9) still holds without (H4) if $f \in \mathscr{S}^{\prime}\left(\mathbb{R}^{d}\right)$. 
Proof: By the conditions (ii), (iii), (iv) and Theorem 4.9, we have for each $t_{0}>0$ that

$$
f\left(X_{T}\right)-f\left(X_{t_{0}}\right)=\sum_{i=1}^{d} \int_{t_{0}}^{T}\left(A_{i} f\right)\left(X_{t}\right) \mathrm{d} w^{i}(t)+\int_{t_{0}}^{T}(L f)\left(X_{t}\right) \mathrm{d} t
$$

in $\mathbb{D}^{-\infty}$. Letting $t_{0} \downarrow 0$ with using (i), we have $f\left(X_{t_{0}}\right) \rightarrow f(x)$ in probability. Moreover, again by (i), we can take $\delta>0$ such that for any $y \in \mathbb{R}^{d},|y-x|<\delta$ implies $|f(y)|<|f(x)|+1$. Thus $\mathbf{E}\left[f\left(X_{t}\right)^{4}\right] \leqslant(|f(x)|+1)^{4}+\mathbf{E}\left[f\left(X_{t}\right)^{4} ;\left|X_{t}-x\right|>\delta\right]$, where $\lim \sup _{t \downarrow 0} \mathbf{E}\left[f\left(X_{t}\right)^{4} ;\left|X_{t}-x\right|>\delta\right]<+\infty$ in view of (ii) and Lemma 3.10(ii). Therefore $\left\{f\left(X_{t}\right)^{2}\right\}_{0<t \leqslant T}$ is $L_{2}$-bounded, so that $\left\{\left(f\left(X_{t}\right)-f(x)\right)^{2}\right\}_{0<t \leqslant T}$ is uniformly integrable. Hence $f\left(X_{t_{0}}\right) \rightarrow f(x)$ in $L_{2}$ as $t_{0} \downarrow 0$ and (4.9) is proved.

If $f \in \mathscr{S}^{\prime}\left(\mathbb{R}^{d}\right)$, then $f$ has at most polynomial growth. In this case, $\left\{f\left(X_{t}\right)^{2}\right\}_{0<t \leqslant T}$ is $L_{2}$-bounded without assuming (H4) and then (4.9) can be proved similarly.

By Proposition 3.12, Lemma 3.11 and Theorem 4.9, we obtain the following.

Corollary 4.11. Let $x \in \mathbb{R}^{d}$ and $\Lambda \in \mathscr{E}^{\prime}\left(\mathbb{R}^{d}\right)$ be such that $\operatorname{supp} \Lambda \not \supset x$. Assume (H1), (H2) and (H4). Then we have in $\mathbb{D}^{-\infty}$,

$$
\Lambda(X(T, x, w))=\sum_{i=1}^{d} \int_{0}^{T}\left(A_{i} \Lambda\right)(X(t, x, w)) \mathrm{d} w^{i}(t)+\int_{0}^{T}(L \Lambda)(X(t, x, w)) \mathrm{d} t .
$$

This still holds without (H4) if $f \in \mathscr{S}^{\prime}\left(\mathbb{R}^{d}\right)$.

4.3. An application and examples. In the sequel, we denote by $X=\left(X_{t}\right)_{t \geqslant 0}$ the unique strong solution to the $d$-dimensional stochastic differential equation

$$
\mathrm{d} X_{t}=\sigma\left(X_{t}\right) \mathrm{d} w(t)+b\left(X_{t}\right) \mathrm{d} t, \quad X_{0}=x \in \mathbb{R}^{d},
$$

where $w=(w(t))_{t \geqslant 0}$ is a $d$-dimensional Wiener process. We denote by $L$ the associated generator, i.e., $L=\frac{1}{2} \sum_{i, j=1}^{d}\left(\sigma \sigma^{*}\right)_{j}^{i} \partial_{i} \partial_{j}+\sum_{i=1}^{d} b^{i} \partial_{i}$.

Let $\left\{F_{\varepsilon}\right\}_{\varepsilon \in I} \subset \mathbb{D}^{\infty}\left(\mathbb{R}^{d}\right)$ be a bounded and uniformly non-degenerate family (see Definition 2.1). Here, the boundedness is used in the sense of $\left\{F_{\varepsilon}\right\}_{\varepsilon \in I}$ is bounded in $\mathbb{D}_{p}^{k}\left(\mathbb{R}^{d}\right)$ for each $p \in(1, \infty)$ and $k \in \mathbb{Z}_{\geqslant 0}$.

Lemma 4.12. For any $s \in \mathbb{R}, p \in(1, \infty)$ and $p^{\prime}>p$, there exists $c=c\left(s, p, p^{\prime}\right)>0$ such that

$$
\left\|\Lambda\left(F_{\varepsilon}\right)\right\|_{p, s} \leqslant c\left\|(1-\triangle)^{s / 2} \Lambda\right\|_{L_{p^{\prime}}\left(\mathbb{R}^{d}, \mathrm{~d} x\right)}
$$

for every $\varepsilon \in I$ and $\Lambda \in \mathscr{S}^{\prime}\left(\mathbb{R}^{d}\right)$ with $(1-\triangle)^{s / 2} \Lambda \in L_{p^{\prime}}\left(\mathbb{R}^{d}, \mathrm{~d} x\right)$.

We note that one can take $p^{\prime}=p$ when $F_{\varepsilon}=\varepsilon^{-1} w\left(\varepsilon^{2} T\right)$ as mentioned in Remark A.5. We don't give a proof of Lemma 4.12. However, we prove a similar inequality (Lemma A.4) in Section A.3. The same techniques there are available to show Lemma 4.12 if we replace the harmonic oscillator $H$ by the Bessel potential $(1-\triangle)$. (Although the step (b) in the proof of Lemma A.4 uses results by Bongioanni and Torrea (2006), we don't need for the proof of Lemma 4.12. The steps (a) and (b) for the proof of Lemma 4.12 can be established analogously by a standard integrationby-parts techniques in the Malliavin calculus. The steps (c)-(e) also run in exactly the same way.)

Let $H_{p}^{s}\left(\mathbb{R}^{d}\right):=(1-\triangle)^{-s / 2} L_{p}\left(\mathbb{R}^{d}, \mathrm{~d} z\right), p \in(1, \infty), s \in \mathbb{R}$ be the Bessel potential spaces (see Abels, 2012 and Krylov, 2008 for details). By imitating the proof of 
Corollary 2.8 with using Lemma 4.12 (instead of Proposition 2.6), we can show that for each $p \in(1, \infty), s \in \mathbb{R}$ and $\Lambda \in H_{p}^{s}\left(\mathbb{R}^{d}\right)$, the mapping $(0, \infty) \ni t \mapsto \Lambda\left(X_{t}\right) \in \mathbb{D}_{p^{\prime}}^{s}$ is continuous for $p^{\prime} \in(1, p)$ under assumptions (H1) and (H2).

We are now in a position to prove Corollary 1.6.

Proof of Corollary 1.6: (i) is clear by Lemma 4.12.

(ii) The operator $L$ is a uniformly elliptic operator of the second order satisfying (H4). Hence the elliptic regularity theorem (see e.g., Abels, 2012, Chapter III, Section 7.3, Theorem 7.13) assures that $f:=(1-L)^{-1} \Lambda \in H_{p}^{s+2}\left(\mathbb{R}^{d}\right)$ and $\sigma_{j}^{k} \partial_{k} f \in$ $H_{p}^{s+1}\left(\mathbb{R}^{d}\right)$ for each $j, k=1,2, \cdots, d$. On the other hand, Theorem 4.9 gives

$$
f\left(X_{T}\right)-f\left(X_{t_{0}}\right)=\sum_{j, k=1}^{d} \int_{t_{0}}^{T}\left(\sigma_{j}^{k} \partial_{k} f\right)\left(X_{t}\right) \mathrm{d} w^{j}(t)+\int_{t_{0}}^{T}(L f)\left(X_{t}\right) \mathrm{d} t .
$$

Let $p^{\prime} \in[2, p)$ be arbitrary. We find that $f\left(X_{T}\right) \in \mathbb{D}_{p^{\prime}}^{s+2}$ by (i) and $\int_{t_{0}}^{T}\left(\sigma_{j}^{k} \partial_{k} f\right)\left(X_{t}\right) \mathrm{d} w^{j} \in \mathbb{D}_{p^{\prime}}^{s+1}$ for $T>0$ by Theorem 4.3, so that we have $\int_{t_{0}}^{T}(L f)\left(X_{t}\right) \mathrm{d} t \in \mathbb{D}_{p^{\prime}}^{s+1}$. Furthermore, since $\left[t_{0}, T\right] \ni t \mapsto f\left(X_{t}\right) \in \mathbb{D}_{p^{\prime}}^{s+2}$ is continuous (recall the remark just before the proof), this mapping is Bochner integrable and $\int_{t_{0}}^{T} f\left(X_{t}\right) \mathrm{d} t \in \mathbb{D}_{p^{\prime}}^{s+2}$. Therefore

$$
\int_{t_{0}}^{T} \Lambda\left(X_{t}\right) \mathrm{d} t=\int_{t_{0}}^{T} f\left(X_{t}\right) \mathrm{d} t-\int_{t_{0}}^{T}(L f)\left(X_{t}\right) \mathrm{d} t \in \mathbb{D}_{p^{\prime}}^{s+1} .
$$

Remark 4.13. By the above remark (just after Lemma 4.12), one would see that in Corollary 1.6, we can take $p^{\prime}=p$ when $\sigma=$ (identity matrix) and $b=0$, i.e., $X_{t}=w(t)$.

Second, we investigate the class $\mathbb{D}_{p}^{s}$ to which $\int_{t_{0}}^{T} \Lambda\left(X_{t}\right) \mathrm{d} t$ belongs when $t_{0}=0$ for several cases of $\Lambda \in \mathscr{S}^{\prime}\left(\mathbb{R}^{d}\right)$.

Example 4.14. Assume $d=1,(\mathrm{H} 1),(\mathrm{H} 3)$ and (H4). Let

$$
\begin{aligned}
& s(x):=\int_{0}^{x} \exp \left\{-\int_{0}^{z} \frac{2 b(\eta)}{\sigma(\eta)^{2}} \mathrm{~d} \eta\right\} \mathrm{d} z, \\
& m(x):=2 \int_{0}^{x} \exp \left\{\int_{0}^{z} \frac{2 b(\eta)}{\sigma(\eta)^{2}} \mathrm{~d} \eta\right\} \frac{\mathrm{d} z}{\sigma(z)^{2}},
\end{aligned} \quad \text { for } x \in \mathbb{R} .
$$

The function $s(x)$ is called the scale function of $L$ and the measure $m(\mathrm{~d} x)=$ $m^{\prime}(x) \mathrm{d} x$ is called the speed measure of $L$. We fix $y \in \mathbb{R}$ and define $u: \mathbb{R} \rightarrow \mathbb{R}$ by

$$
u(x):=: u(x, y):=\frac{m^{\prime}(y)}{2}|s(x)-s(y)|, \quad x \in \mathbb{R} .
$$

Then it is easily checked that $L u=\delta_{y}$ and

$$
(A u)(x)=\operatorname{sgn}(x-y)\left[\exp \left\{-\int_{y}^{x} \frac{2 b(\eta)}{\sigma(\eta)^{2}} \mathrm{~d} \eta\right\} \frac{\sigma(x)}{\sigma(y)^{2}}\right], \quad x \in \mathbb{R} .
$$

in the distributional sense. Now we shall prove

$$
\left\{\begin{array} { l } 
{ s < \frac { 1 } { 2 } ; } \\
{ p \in ( 1 , \frac { 1 } { s } ) }
\end{array} \Rightarrow \left\{\begin{array}{l}
1_{\{X(t, x, w)<y\}} \in \mathbb{D}_{p}^{s} \\
\lim \sup _{t \downarrow 0}\left\|1_{\{X(t, x, w)<y\}}\right\|_{p, s}<\infty
\end{array}\right.\right.
$$


In fact, let $s \in(0,1 / 2)$ and $p \in\left(1, \frac{1}{s}\right)$. Take $p^{\prime}>p$ so that $s p^{\prime}<1$. Then by Proposition 3.7 and Lemma A.4, there exists $c>0$ such that

$$
\begin{aligned}
& \left\|1_{\{X(t, x, w)<y\}}\right\|_{p, s}=\left\|1_{(-\infty,(y-x) / \sqrt{t})}\left(\widetilde{F}_{t}\right)\right\|_{p, s} \\
& \quad \leqslant c\left\|\left(z^{2}-\triangle\right)^{s / 2} 1_{(-\infty,(y-x) / \sqrt{t})}\right\|_{L_{p^{\prime}}(\mathbb{R}, \mathrm{d} z)} \quad \text { for all } t \in(0, T] .
\end{aligned}
$$

where $\widetilde{F}_{t}=\left(X^{\sqrt{t}}(1, x, w)-x\right) / \sqrt{t}$. Hence we can conclude (4.12) by Proposition A.3-(iii).

By the condition $(\mathrm{H} 3)$ and $(\mathrm{H} 4)$, we see that $u \in \mathscr{E}^{\prime}(\mathbb{R})$. Hence by Theorem 3.4 and Theorem 4.10, we have

$$
\int_{0}^{T} \delta_{y}\left(X_{t}\right) \mathrm{d} t=u\left(X_{T}\right)-u(x)-\int_{0}^{T}(A u)\left(X_{t}\right) \mathrm{d} w(t) .
$$

It is easy to see that $u\left(X_{T}\right) \in \cap_{p>1} \mathbb{D}_{p}^{1}$ and by (H3) that

$$
\exp \left\{-\int_{y}^{X_{t}} \frac{2 b(\eta)}{\sigma(\eta)^{2}} \mathrm{~d} \eta\right\} \frac{\sigma\left(X_{t}\right)}{\sigma(y)^{2}} \in \mathbb{D}_{p}^{\infty} \quad \text { for all } p \in(1, \infty)
$$

From this and (4.12), we find $\lim \sup _{t \downarrow 0}\left\|(A u)\left(X_{t}\right)\right\|_{p, s}<\infty$ for $s<1 / 2$ and $p \in$ $\left(1, \frac{1}{s}\right)$. Then by Theorem 4.3-(ii), $\int_{0}^{T}(A u)\left(X_{t}\right) \mathrm{d} w(t) \in \mathbb{D}_{p}^{s}$ for $s<\frac{1}{2}$ and $p \in\left[2, \frac{1}{s}\right)$, and thus we reached: under (H1), (H3) and (H4),

$$
\int_{0}^{T} \delta_{y}\left(X_{t}\right) \mathrm{d} t \in \mathbb{D}_{p}^{s} \quad \text { for } s<\frac{1}{2} \text { and } p \in\left[2, \frac{1}{s}\right) .
$$

See Airault et al. (2000) for a more general and stronger result.

Remark 4.15. In particular, we see from Example 4.14 that $\int_{0}^{T} \delta_{y}\left(X_{t}\right) \mathrm{d} t$ is a classical Wiener functional when $d=1$, which is related to the local time at $y$. This can be seen as follows: Classically, the symmetric local time $\{\widetilde{l}(y, t): y \in \mathbb{R}, t \geqslant 0\}$ is defined as a unique increasing process such that

$$
\left|X_{t}-y\right|=|x-y|+\int_{0}^{t} \operatorname{sgn}\left(X_{s}-y\right) \mathrm{d} X_{s}+\widetilde{l}(y, t)
$$

and equivalently given by

$$
\widetilde{l}(y, t)=\lim _{\varepsilon \downarrow 0} \frac{1}{2 \varepsilon} \int_{0}^{t} 1_{(y-\varepsilon, y+\varepsilon)}\left(X_{s}\right) \mathrm{d}\langle X\rangle_{s}
$$

(see Revuz and Yor, 1999). Since it holds $\lim _{\varepsilon \downarrow 0}(2 \varepsilon)^{-1} \sigma^{2} 1_{(y-\varepsilon, y+\varepsilon)}=\sigma \delta_{y}=\sigma(y) \delta_{y}$ in $\mathscr{S}^{\prime}(\mathbb{R})$, we have

$$
\widetilde{l}(y, t)=\sigma(y)^{2} \int_{0}^{t} \delta_{y}\left(X_{u}\right) \mathrm{d} u .
$$

In the sequel, we denote $\mathbb{D}_{2}^{s-}:=\cap_{\varepsilon>0} \mathbb{D}_{2}^{s-\varepsilon}$.

Example 4.16. Assume $d=1,(\mathrm{H} 1)$, (H3) and (H4). Let $x, y \in \mathbb{R}$ be such that $x \neq y$. By using Lemma 4.12, we see that the mapping $(0, T] \ni t \mapsto \delta_{y}^{\prime}(X(t, x, w)) \in \mathbb{D}_{2}^{-k}$ is continuous for some $k \in \mathbb{Z}_{\geqslant 0}$.

Define $u\left(z_{1}, z_{2}\right)$ by $(4.11)$, and then

$$
\begin{aligned}
& v(z):=-\left(\partial_{z_{2}} u\right)(z, y) \\
& =\frac{1}{2}\left\{m^{\prime}(y) s^{\prime}(y) \operatorname{sgn}(z-y)-m^{\prime \prime}(y)|s(z)-s(y)|\right\} \in \mathscr{E}^{\prime}(\mathbb{R})
\end{aligned}
$$


satisfies $L v=\delta_{y}^{\prime}$ and

$$
(A v)(z)=\frac{\sigma(z)}{2}\left(2 m^{\prime}(y) s^{\prime}(y) \delta_{y}(z)-m^{\prime \prime}(y) s^{\prime}(z) \operatorname{sgn}(z-y)\right) .
$$

Since $X_{t}=X(t, x, w)$ is non-degenerate for each $t>0$, it is known by Watanabe (1991) that $\delta_{y}\left(X_{t}\right) \in \mathbb{D}_{p}^{s}$ if $s \in\left(-1,-\frac{1}{2}\right)$ and $p \in\left(1, \frac{1}{1+s}\right)$. Hence by virtue of conditions $(\mathrm{H} 1)$ and $(\mathrm{H} 3)$, for each $t_{0} \in(0, T)$ and $p \geqslant 2$, we find $\int_{t_{0}}^{T}(A u)\left(X_{t}\right) \mathrm{d} w(t) \in$ $\mathbb{D}_{p}^{\left(\frac{1}{p}-1\right)-}$, and thus

$$
\int_{t_{0}}^{T} \delta_{y}^{\prime}\left(X_{t}\right) \mathrm{d} t \in \mathbb{D}_{p}^{\left(\frac{1}{p}-1\right)-} \text { for any } t_{0}>0 \text { and } p \geqslant 2
$$

Example 4.17. In the above Example 4.16, we shall try to investigate the class to which $\int_{0}^{T} \delta_{y}^{\prime}\left(X_{t}\right) \mathrm{d} t$ belongs. Let $p \geqslant 2$ be arbitrary. Since $\delta_{y}(x+\varepsilon \bullet)=$ $\lim _{a \downarrow 0}(2 \pi a)^{-1 / 2} \exp \left\{-(x+\varepsilon \bullet-y)^{2} /(2 a)\right\}=\varepsilon^{-1} \lim _{a \downarrow 0}\left(2 \pi \varepsilon^{-2} a\right)^{-1 / 2} \exp \left\{-\left(\frac{y-x}{\varepsilon}-\right.\right.$ $\left.\bullet)^{2} /\left(2 \varepsilon^{-2} a\right)\right\}=\varepsilon^{-1} \delta_{(y-x) / \varepsilon}(\bullet)$ in the distributional sense, we have

$$
\sigma\left(X_{t}\right)\left(m^{\prime} s^{\prime}\right)(y) \delta_{y}\left(X_{t}\right)=\left(\sigma m^{\prime} s^{\prime}\right)(y) \varepsilon^{-1} \delta_{(y-x) / \varepsilon}\left(\widetilde{F}_{\varepsilon}\right),
$$

where $\varepsilon:=\sqrt{t}, X_{t}=X(t, x, w)$ and $\widetilde{F}_{\varepsilon}=\left(X_{t}-x\right) / \varepsilon$. We shall prove

$$
\varepsilon^{-1}\left\|\delta_{(y-x) / \varepsilon}\left(\widetilde{F}_{\varepsilon}\right)\right\|_{p, s} \rightarrow 0 \quad \text { as } \varepsilon=\sqrt{t} \downarrow 0 \text { for every } s<-1 \text {. }
$$

Note that the probability density function $p_{\widetilde{F}_{\varepsilon}}$ of $\widetilde{F}_{\varepsilon}$ has an estimate $\sup _{0<\varepsilon \leqslant 1} p_{\widetilde{F}_{\varepsilon}}(z)$ $\leqslant C p(z)$ for some $C>0$, where $p(z):=\left(2 \pi c^{\prime}\right)^{-1 / 2} \mathrm{e}^{-z^{2} /\left(2 c^{\prime}\right)}$ and $c^{\prime}>0$ is a constant. By refining the argument in the proof of Lemma A.4, one finds that for every $p^{\prime}>p$,

$$
\left\|\delta_{(y-x) / \varepsilon}\left(\widetilde{F}_{\varepsilon}\right)\right\|_{p, s} \leqslant \text { const. }\left\|(1-\triangle)^{s / 2} \delta_{(y-x) / \varepsilon}\right\|_{L_{p^{\prime}}(\mathbb{R}, \mu)}
$$

for all $\varepsilon \in(0,1]$, where $\mu(\mathrm{d} z)=p(z) \mathrm{d} z$. To estimate the last quantity, we apply the Fourier transformation and integration by parts to get

$$
\begin{aligned}
& \varepsilon^{-2}(1-\triangle)^{s / 2} \delta_{(y-x) / \varepsilon}(z)=\frac{-1}{2 \pi} \int_{-\infty}^{\infty} \frac{\mathrm{e}^{i \xi z}}{\left(1+\xi^{2}\right)^{-s / 2}} \varepsilon^{-2} \mathrm{e}^{i \xi \frac{y-x}{\varepsilon}} \mathrm{d} \xi \\
& =\frac{1}{2 \pi(y-x)^{2}} \int_{-\infty}^{\infty}\left\{\frac{\mathrm{d}^{2}}{\mathrm{~d} \xi^{2}} \frac{\mathrm{e}^{i \xi z}}{\left(1+\xi^{2}\right)^{-s / 2}}\right\} \mathrm{e}^{i \xi \frac{y-x}{\varepsilon}} \mathrm{d} \xi
\end{aligned}
$$

in which, one finds that there exists $c=c(s)>0$ such that

$$
\left|\frac{\mathrm{d}^{2}}{\mathrm{~d} \xi^{2}} \frac{\mathrm{e}^{i \xi z}}{\left(1+\xi^{2}\right)^{-s / 2}}\right| \leqslant c\left(1+z^{2}\right)\left(1+\xi^{2}\right)^{s / 2}
$$

Hence we obtain

$$
\left|\varepsilon^{-2}(1-\triangle)^{s / 2} \delta_{(y-x) / \varepsilon}(z)\right| \leqslant \frac{c}{2 \pi(y-x)^{2}}\left(1+z^{2}\right) \int_{-\infty}^{\infty}\left(1+\xi^{2}\right)^{s / 2} \mathrm{~d} \xi
$$

Note that $\int_{-\infty}^{\infty}\left(1+\xi^{2}\right)^{s / 2} \mathrm{~d} \xi<+\infty$ since $s<-1$. We thus have

$$
\sup _{\varepsilon>0} \varepsilon^{-2 p^{\prime}}\left\|(1-\triangle)^{s / 2} \delta_{(y-x) / \varepsilon}\right\|_{L_{p^{\prime}}(\mathbb{R}, \mu)}^{p^{\prime}} \leqslant c^{\prime} \int_{\mathbb{R}}\left(1+z^{2}\right)^{p^{\prime}} p(z) \mathrm{d} z<+\infty,
$$

where the constant $c^{\prime}>0$ may depend on $y-x$, and which gives (4.13). 
By (H1), (H3) and (H4), we see that $\lim _{t \downarrow 0} \sigma\left(X_{t}\right)=\sigma(x)$ in $\mathbb{D}^{\infty}$. Combining this with (4.13) and Theorem 4.3-(i), we obtain $\int_{0}^{T}\left(\sigma \delta_{y}\right)\left(X_{t}\right) \mathrm{d} w(t) \in \mathbb{D}_{p}^{(-1)-}$. On the other hand, we have $\int_{0}^{T}\left(\sigma s^{\prime}\right)\left(X_{t}\right) \operatorname{sgn}\left(X_{t}-y\right) \mathrm{d} w(t) \in L_{2} \subset \mathbb{D}_{p}^{(-1)-}$. Therefore

$$
\begin{aligned}
& \int_{0}^{T}(A u)\left(X_{t}\right) \mathrm{d} w(t) \\
& =m^{\prime}(y) s^{\prime}(y) \int_{0}^{T}\left(\sigma \delta_{y}\right)\left(X_{t}\right) \mathrm{d} w(t)-\frac{m^{\prime \prime}(y)}{2} \int_{0}^{T}\left(\sigma s^{\prime}\right)\left(X_{t}\right) \operatorname{sgn}\left(X_{t}-y\right) \mathrm{d} w(t) \in \mathbb{D}_{p}^{(-1)-} .
\end{aligned}
$$

Hence by Theorem 4.10,

$$
\int_{0}^{T} \delta_{y}^{\prime}\left(X_{t}\right) \mathrm{d} t \in \mathbb{D}_{p}^{(-1)-} \quad \text { for any } p \geqslant 2 .
$$

Example 4.18 (Cauchy's principal value of $1 / x$ ). Let $d=1$. The tempered distribution p.v. $\frac{1}{x}$ is defined by

$$
\left\langle\text { p.v. } \frac{1}{x}, f\right\rangle:=\lim _{\varepsilon \downarrow 0} \int_{|x|>\varepsilon} \frac{f(x)}{x} \mathrm{~d} x, \quad f \in \mathscr{S}(\mathbb{R}) .
$$

Let $\Lambda \in \mathscr{S}^{\prime}(\mathbb{R})$ be the regular distribution (i.e., a distribution associated with a locally integrable function) given by $\Lambda=x \log |x|-x$. Then the distributional derivatives are: $\Lambda^{\prime}=\log |x|$ and $\Lambda^{\prime \prime}=$ p.v. $\frac{1}{x}$.

We shall first show the Bochner integrability of the pull-back of (p.v. $\left.\frac{1}{x}\right)$ by a Brownian motion $w(t):(0, T] \ni t \mapsto\left(\operatorname{p} \cdot v \cdot \frac{1}{x}\right)(w(t))$. For each $J \in \mathbb{D}^{\infty}$ and $p, q>1$ such that $1 / p+1 / q=1$, we obtain

$$
\begin{aligned}
& \mathbf{E}\left[\left(\text { p.v. } \frac{1}{x}\right)(w(t)) J\right]=\mathbf{E}\left[(x \log |x|-x)^{\prime \prime}(w(t)) J\right] \\
& =\mathbf{E}\left[(w(t) \log |w(t)|-w(t)) l_{t}(J)\right] \\
& \leqslant\|(w(t) \log |w(t)|-w(t))\|_{p}\left\|l_{t}(J)\right\|_{q} .
\end{aligned}
$$

Here $l_{t}(J)=\sum_{i=0}^{2}\left\langle P_{i}(t), D^{i} J\right\rangle_{H^{\otimes i}} \in \mathbb{D}^{\infty}$ for some $P_{i}(t) \in \mathbb{D}^{\infty}\left(H^{\otimes i}\right), i=0,1,2$ which are polynomials in $w(t)$, its derivatives and $\|D w(t)\|_{H}^{-2}=t^{-1}$. Since we have

$$
\begin{aligned}
& \|w(t) \log |w(t)|\|_{p}^{p}=\int_{\mathbb{R}}|(\sqrt{t} x) \log (\sqrt{t}|x|)|^{p} \frac{\mathrm{e}^{-x^{2} / 2}}{\sqrt{2 \pi}} \mathrm{d} x \\
& \leqslant 2^{p}|\sqrt{t} \log \sqrt{t}|^{p} \int_{\mathbb{R}}|x|^{p} \frac{\mathrm{e}^{-x^{2} / 2}}{\sqrt{2 \pi}} \mathrm{d} x+2^{p} t^{p / 2} \int_{\mathbb{R}}|x \log | x||^{p} \frac{\mathrm{e}^{-x^{2} / 2}}{\sqrt{2 \pi}} \mathrm{d} x,
\end{aligned}
$$

which tends to zero as $t \downarrow 0$, and hence $\lim \sup _{t \downarrow 0} \|\left(\right.$ p.v. $\left.\frac{1}{x}\right)(w(t)) \|_{p,-2}<+\infty$ for $p \geqslant 2$. This proves that $(0, T] \ni t \mapsto\left(\right.$ p.v. $\left.\frac{1}{x}\right)(w(t)) \in \mathbb{D}_{2}^{-2}$ is Bochner integrable. On the other hand, it is clear that $\int_{0}^{T}\|\log |w(t)|\|_{2}^{2} \mathrm{~d} t<+\infty$.

Now, by Theorem 4.10, we have

$$
w(T) \log |w(T)|=\int_{0}^{T} \log |w(t)| \mathrm{d} w(t)+\frac{1}{2} \int_{0}^{T}\left(\text { p.v. } \frac{1}{x}\right)(w(t)) \mathrm{d} t .
$$

The chaos expansion of $\log |w(1)|$ is given by

$$
\log |w(1)|=\mathbf{E}[\log |w(1)|]+\sum_{n=1}^{\infty} \frac{1}{n !} \lim _{\varepsilon \downarrow 0} \int_{|x| \geqslant \varepsilon} \frac{1}{x} H_{n-1}(x) \frac{\mathrm{e}^{-x^{2} / 2}}{\sqrt{2 \pi}} \mathrm{d} x H_{n}(w(1)) .
$$


Put $\mu(\mathrm{d} x):=(2 \pi)^{-1 / 2} \exp \left(-x^{2} / 2\right) \mathrm{d} x$. It is clear that $\lim _{\varepsilon \downarrow 0} \int_{|x|>\varepsilon} \frac{1}{x} H_{1}(x) \mu(\mathrm{d} x)=$ $\int_{\mathbb{R}} \mu(\mathrm{d} x)=1$. For $n \geqslant 2$, by using Lemma A.1-(ii), we have

$$
\begin{aligned}
& \lim _{\varepsilon \downarrow 0} \int_{|x|>\varepsilon} \frac{1}{x} H_{n}(x) \mu(\mathrm{d} x) \\
& =\lim _{\varepsilon \downarrow 0} \int_{|x|>\varepsilon} \frac{1}{x}\left\{x H_{n-1}(x)-(n-1) H_{n-2}(x)\right\} \mu(\mathrm{d} x) \\
& =\int_{\mathbb{R}} H_{n-1}(x) \mu(\mathrm{d} x)-(n-1) \lim _{\varepsilon \downarrow 0} \int_{|x|>\varepsilon} \frac{1}{x} H_{n-2}(x) \mu(\mathrm{d} x) \\
& =-(n-1) \lim _{\varepsilon \downarrow 0} \int_{|x|>\varepsilon} \frac{1}{x} H_{n-2}(x) \mu(\mathrm{d} x) .
\end{aligned}
$$

Therefore if $n=2 k+1$, then

$$
\begin{aligned}
& \lim _{\varepsilon \downarrow 0} \int_{|x|>\varepsilon} \frac{1}{x} H_{n}(x) \mu(\mathrm{d} x) \\
& =(-2 k)(-(2 k-2))(-(2 k-4)) \cdots(-2) \cdot \lim _{\varepsilon \downarrow 0} \int_{|x|>\varepsilon} \frac{1}{x} H_{1}(x) \mu(\mathrm{d} x) \\
& =(-1)^{k}(2 k) ! !
\end{aligned}
$$

Similarly, if $n$ is even, $\lim _{\varepsilon \downarrow 0} \int_{|x|>\varepsilon} x^{-1} H_{n}(x) \mu(\mathrm{d} x)=0$. Thus we get

$$
\log |w(1)|=\mathbf{E}[\log |w(1)|]+\sum_{n=0}^{\infty}(-1)^{n} \frac{(2 n) ! !}{(2 n+2) !} H_{2 n+2}(w(1)),
$$

from which, we find that

$$
\begin{aligned}
& \|\log |w(t) / \sqrt{t}|\|_{2, s}^{2}=\|\log |w(1)|\|_{2, s}^{2} \\
& =\mathbf{E}[\log |w(1)|]^{2}+\sum_{n=0}^{\infty}(1+n)^{s} \frac{((2 n) ! !)^{2}}{(2 n+2) !} .
\end{aligned}
$$

Noting $(2 n) ! !=2^{n} n !$, the Stirling formula tells us

- $\|\log |w(t) / \sqrt{t}|\|_{2, s}=\|\log |w(1)|\|_{2, s}<+\infty$ iff $s<\frac{1}{2}$.

- A similar computation shows that

$$
\|\left(\text { p.v. } \frac{1}{x}\right)(w(1)) \|_{2, s}<+\infty \quad \text { iff } s<-\frac{1}{2} .
$$

Now, for $s<1 / 2$, we have

$$
\int_{0}^{T}\|\log |w(t)|\|_{2, s}^{2} \mathrm{~d} t \leqslant 4\left(\int_{0}^{T}(\log \sqrt{t})^{2} \mathrm{~d} t+T\|\log |w(1)|\|_{2, s}^{2}\right)<+\infty,
$$

so that $\int_{0}^{T} \log |w(t)| \mathrm{d} w(t) \in \mathbb{D}_{2}^{(1 / 2)-}$ by Theorem 4.3-(ii). Hence by (4.14),

$$
\int_{0}^{T}\left(\text { p.v. } \frac{1}{x}\right)(w(t)) \mathrm{d} t \in \mathbb{D}_{2}^{(1 / 2)-} .
$$

Example 4.19. Let $d \geqslant 2$ and $x, y \in \mathbb{R}^{d}$. Suppose (H1), (H3) and (H4). Since $\delta_{y} \in H_{p}^{s}\left(\mathbb{R}^{d}\right)$ if $s<-(p-1) d / p$ (Lemma A.6), we find from Corollary 1.6,

$$
\int_{t_{0}}^{T} \delta_{y}\left(X_{t}\right) \mathrm{d} t \in \mathbb{D}_{p}^{\left(1-\frac{(p-1) d}{p}\right)-} \text { for } t_{0}>0 \text { and } p \geqslant 2,
$$


where $X_{t}=X(t, x, w)$.

Assume that $x \neq y$, and then we shall further investigate the class to which $\int_{0}^{T} \delta_{y}\left(X_{t}\right) \mathrm{d} t$ belongs. Let $f:=(1-L)^{-1} \delta_{y}$ and let $\phi: \mathbb{R}^{d} \rightarrow \mathbb{R}$ be a $C^{\infty}$-function such that (1) $x \notin \operatorname{supp} \phi,(2) \phi \equiv 1$ on a neighbourhood of $y$, and (3) $\operatorname{supp} \phi$ is compact. By Theorem 4.10, we have

$$
(\phi f)\left(X_{T}\right)-(\phi f)(x)=\sum_{i, j=1}^{d} \int_{0}^{T}\left(\sigma_{j}^{i} \partial_{i}(\phi f)\right)\left(X_{t}\right) \mathrm{d} w^{j}(t)+\int_{0}^{T}(L(\phi f))\left(X_{t}\right) \mathrm{d} t,
$$

in which we note that

$$
L(\phi f)=(L \phi) f+\langle\sigma \nabla \phi, \sigma \nabla f\rangle_{\mathbb{R}^{d}}+\phi f-\delta_{y}
$$

and $(L \phi) f,\langle\sigma \nabla \phi, \sigma \nabla f\rangle_{\mathbb{R}^{d}} \in \mathscr{S}\left(\mathbb{R}^{d}\right)$. By Lemma A.7-(ii),

$$
\left\{\begin{array} { l } 
{ p \in ( 1 , \infty ) ; } \\
{ - \frac { d } { 2 } < s < \operatorname { m i n } \{ \frac { p } { p - 1 } - d , 0 \} }
\end{array} \Rightarrow \left\{\begin{array}{l}
\lim _{t \downarrow 0}\left\|(\phi f)\left(X_{t}\right)\right\|_{p, s}=0 ; \\
\lim _{t \downarrow 0}\left\|\partial_{i}(\phi f)\left(X_{t}\right)\right\|_{p, s}=0
\end{array}\right.\right.
$$

and then we have $\lim _{t \downarrow 0}\left\|\left(\sigma_{j}^{i} \partial_{i}(\phi f)\right)\left(X_{t}\right)\right\|_{p, s}=0$ since $\lim _{t \downarrow 0} \sigma_{j}^{i}\left(X_{t}\right)=\sigma_{j}^{i}(x)$ in $\mathbb{D}^{\infty}$ and $\left\|\left(\sigma_{j}^{i} \partial_{i}(\phi f)\right)\left(X_{t}\right)\right\|_{p, s} \leqslant$ const. $\left\|\sigma_{j}^{i}\left(X_{t}\right)\right\|_{q,-s^{\prime}}\left\|\partial_{i}(\phi f)\left(X_{t}\right)\right\|_{r, s^{\prime}}$ (this follows by taking the dual of Watanabe, 1993, inequality (1.6)), where $q, r \in(1, \infty)$ and $s^{\prime} \in \mathbb{R}$ are such that $1 / p=1 / q+1 / r$ and $s<s^{\prime}<\min \left\{\frac{p}{p-1}-d, 0\right\}\left(\leqslant \min \left\{\frac{q}{q-1}-d, 0\right\}\right)$. Now by Theorem 4.3 , we obtain $\int_{0}^{T}\left(\sigma_{j}^{i} \partial_{i}(\phi f)\right)\left(X_{t}\right) \mathrm{d} w^{j}(t) \in \mathbb{D}_{p}^{s}$ for $p \geqslant 2$, so that

$$
\int_{0}^{T} \delta_{y}\left(X_{t}\right) \mathrm{d} t \in \mathbb{D}_{p}^{\left(\frac{p}{p-1}-d\right)-} \text { for any } d \geqslant 2 \text { and } p \geqslant 2 .
$$

Remark 4.20. In Examples 4.16 and 4.19, the conclusions seem not the best possible given $p \geqslant 2$, because recalling that $\int_{t_{0}}^{T} \delta_{y}^{\prime}\left(X_{t}\right) \mathrm{d} t$ and $\int_{t_{0}}^{T} \delta_{y}\left(X_{t}\right) \mathrm{d} t$ belong to $\mathbb{D}_{p}^{\left(\frac{1}{p}-1\right)-}$ and $\mathbb{D}_{p}^{\left(1-\frac{(p-1) d}{p}\right)-}$ respectively for each $t_{0}>0$ (Note that $X_{t}$ 's in each case are different though we are using the same symbol), it is natural to ask that $\int_{0}^{T} \delta_{y}^{\prime}\left(X_{t}\right) \mathrm{d} t$ and $\int_{0}^{T} \delta_{y}\left(X_{t}\right) \mathrm{d} t$ also do. To get further results for $p \in(1,2)$, one would have to investigate Theorem 4.3 for $p \in(1,2)$, which we could not.

\section{Appendix A. Auxiliary Lemmas}

A.1. Some knowledge of Hermite polynomials. The Hermite polynomials $H_{n}, n \in$ $\mathbb{Z}_{\geqslant 0}$ is defined by $H_{0}(x)=1$ and $H_{n}(x):=\partial^{* n} 1(x)$ for $n \in \mathbb{N}$ and $x \in \mathbb{R}$, where

$$
\partial^{*} f(x):=-f^{\prime}(x)+x f(x), \quad x \in \mathbb{R}
$$

for any differentiable function $f: \mathbb{R} \rightarrow \mathbb{R}$. The Hermite functions are now defined by

$$
\phi_{n}(x):=H_{n}(\sqrt{2} x) \mathrm{e}^{-\frac{x^{2}}{2}}, \quad x \in \mathbb{R} \text { and } n \in \mathbb{Z}_{\geqslant 0} .
$$

Some facts about $\left\{H_{n}\right\}_{n=0}^{\infty}$ and $\left\{\phi_{n}\right\}_{n=0}^{\infty}$ are summarized as follows.

Lemma A.1. (i) For each $n \in \mathbb{Z}_{\geqslant 0}$,

$$
H_{n}(x)=(-1)^{n} \mathrm{e}^{\frac{x^{2}}{2}} \frac{\mathrm{d}^{n}}{\mathrm{~d} x^{n}} \mathrm{e}^{-\frac{x^{2}}{2}}=\frac{(-1)^{n} \mathrm{e}^{\frac{x^{2}}{2}}}{\sqrt{2 \pi}} \int_{-\infty}^{+\infty}(i \xi)^{n} \mathrm{e}^{-\frac{\xi^{2}}{2}} \mathrm{e}^{i \xi x} \mathrm{~d} \xi .
$$

(ii) $H_{n}^{\prime}=n H_{n-1}$ and $H_{n}(x)=x H_{n-1}(x)-(n-1) H_{n-2}(x)$.

(iii) $\left\{\frac{1}{\sqrt{n !}} H_{n}\right\}_{n \geqslant 0}$ is a complete orthonormal basis of $L_{2}\left(\mathbb{R},(2 \pi)^{-1 / 2} \mathrm{e}^{-x^{2} / 2} \mathrm{~d} x\right)$. 
(iv) $\left\{(\sqrt{\pi} n !)^{-1 / 2} \phi_{n}\right\}_{n \geqslant 0}$ is a complete orthonormal system of $L_{2}(\mathbb{R}, \mathrm{d} x)$.

(v) $\left(1+x^{2}-\triangle\right) \phi_{n}=2(n+1) \phi_{n}$ for $n \in \mathbb{Z}_{\geqslant 0}$, where $\triangle=\frac{\mathrm{d}^{2}}{\mathrm{~d} x^{2}}$.

A.2. Some knowledge of Heaviside function. We begin with introducing some results by Bongioanni and Torrea (2006). Let $H:=x^{2}-\triangle, A:=\frac{\mathrm{d}}{\mathrm{d} x}+x$ and $B:=-\frac{\mathrm{d}}{\mathrm{d} x}+x$. From the point of view of Lemma A.1-(iii), (iv), A family of linear operators $(\lambda+H)^{s}, s \in \mathbb{R}$ and $\lambda \geqslant 0$ can also be defined by the relation $(\lambda+H)^{s} \phi_{n}=\left(2\left(n+\frac{\lambda+1}{2}\right)\right)^{s} \phi_{n}$ for $n \in \mathbb{Z}_{\geqslant 0}$.

Then for $s<0$, the operator $H^{s / 2}$ has an integral representation (see Bongioanni and Torrea, 2006, Proposition 2)

$$
\left(H^{s / 2} f\right)(x)=\int_{\mathbb{R}} K_{s / 2}(x, y) f(y) \mathrm{d} y \quad \text { for } f \in \mathscr{S}(\mathbb{R}),
$$

where the kernel $K_{s / 2}(x, z)$ has an estimate $K_{s / 2}(x, y) \leqslant c \Phi_{s / 2}(|x-y|)$ for some constant $c>0$ and

$$
\Phi_{s / 2}(x)= \begin{cases}|x|^{-(1+s)} 1_{\{|x|<1\}}+\mathrm{e}^{-x^{2} / 4} 1_{\{|x| \geqslant 1\}} & \text { if } s>-1 \\ (1-\log |x|) 1_{\{|x|<1\}}+\mathrm{e}^{-x^{2} / 4} 1_{\{|x| \geqslant 1\}} & \text { if } s=-1 \\ 1_{\{|x|<1\}}+\mathrm{e}^{-x^{2} / 4} 1_{\{|x| \geqslant 1\}} & \text { if } s<-1\end{cases}
$$

Lemma A.2. If $s \in\left(-1,-\frac{1}{2}\right)$ and $p \in\left(1, \frac{1}{1+s}\right), H^{s / 2} \delta_{y}(x) \in L_{p}(\mathbb{R}, \mathrm{d} x)$.

Proof: Since $s>-1$, we have

$$
\begin{aligned}
\left(H^{s / 2} \delta_{y}\right)(x) & =K_{s / 2}(x, y) \\
& \leqslant c\left(|x-y|^{-(1+s)} 1_{\{|x-y|<1\}}+\mathrm{e}^{-\frac{|x-y|^{2}}{4}} 1_{\{|x-y| \geqslant 1\}}\right) .
\end{aligned}
$$

Hence we can easily conclude the result.

By using Bongioanni and Torrea (2006, Theorem 4, Lemma 4, Theorem 7) and $L_{p}$-multiplier theorem (see Thangavelu, 1993, Chapter 4, Section 4.2, Theorem 4.2.1) for operators $\left[H^{-1}(H+2)\right]^{s / 2}, s \in \mathbb{R}$, we can deduce that for each $s \in \mathbb{R}$, there exists a constant $c_{1}=c_{1}(s)>0$ such that

$$
\begin{aligned}
& \left\|H^{(s+1) / 2} f\right\|_{L_{p}(\mathbb{R}, \mathrm{d} x)} \\
& \leqslant c_{1}\left(\left\|H^{s / 2} \phi\right\|_{L_{p}(\mathbb{R}, \mathrm{d} x)}+\left\|x H^{s / 2} f\right\|_{L_{p}(\mathbb{R}, \mathrm{d} x)}+\left\|H^{s / 2} f\right\|_{L_{p}(\mathbb{R}, \mathrm{d} x)}\right)
\end{aligned}
$$

for all $f=f(x) \in \mathscr{S}(\mathbb{R})$, where $\phi:=A f$. By a standard argument, this inequality extends and is still valid for $f=f(x) \in \mathscr{S}^{\prime}(\mathbb{R})$ such that $\left(H^{s / 2} A f\right),\left(H^{s / 2} f\right)$, $\left(x H^{s / 2} f\right) \in L_{p}(\mathbb{R}, \mathrm{d} x)$.

Proposition A.3. Let $y \in \mathbb{R}$. For each $s<1 / 2$ and $p \in(1,1 / s)$, we have

(i) $H^{s / 2} 1_{(-\infty, y)} \in L_{p}(\mathbb{R}, \mathrm{d} x)$,

(ii) $\lim _{y \rightarrow-\infty}\left\|H^{s / 2} 1_{(-\infty, y)}\right\|_{L_{p}(\mathbb{R}, \mathrm{d} x)}=0$ and

(iii) $\sup _{y \in \mathbb{R}}\left\|H^{s / 2} 1_{(-\infty, y)}\right\|_{L_{p}(\mathbb{R}, \mathrm{d} x)}<\infty$. 
Proof: Let $f(x):=1_{(-\infty, y)}(x)$ and $\phi(x):=A f(x)=\delta_{y}(x)+x 1_{(-\infty, y)}(x)$. Then by (A.2), we have

$$
\begin{aligned}
& \left\|H^{s / 2} 1_{(-\infty, y)}\right\|_{L_{p}(\mathbb{R}, \mathrm{d} x)} \\
& \leqslant 2 c_{1}\left(\left\|H^{(s-1) / 2} \delta_{y}\right\|_{L_{p}(\mathbb{R}, \mathrm{d} x)}+\left\|H^{(s-1) / 2}\left(x 1_{(-\infty, y)}\right)\right\|_{L_{p}(\mathbb{R}, \mathrm{d} x)}\right. \\
& \left.\quad+\left\|x H^{(s-1) / 2} 1_{(-\infty, y)}\right\|_{L_{p}(\mathbb{R}, \mathrm{d} x)}+\left\|H^{(s-1) / 2} 1_{(-\infty, y)}\right\|_{L_{p}(\mathbb{R}, \mathrm{d} x)}\right),
\end{aligned}
$$

which is finite by virtue of Lemma A.2 and by a similar argument in Lemma A.2 with using the integral expression (A.1). Furthermore again by (A.1), we find also that the last four terms converge to zero as $y \rightarrow-\infty$, and uniformly bounded in $y \in \mathbb{R}$.

A.3. Fractional inequalities. Let $\left\{F_{\varepsilon}\right\}_{\varepsilon \in I} \subset \mathbb{D}^{\infty}$ be a bounded and uniformly nondegenerate family (see Definition 2.1). Here, the boundedness is used in the sense of $\left\{F_{\varepsilon}\right\}_{\varepsilon \in I}$ is bounded in $\mathbb{D}_{p}^{k}$ for each $p \in(1, \infty)$ and $k \in \mathbb{Z}_{\geqslant 0}$.

Lemma A.4. For any $s \in \mathbb{R}, p \in(1, \infty)$ and $p^{\prime}>p$, there exists $c=$ $c\left(s, p, p^{\prime},\left\{F_{\varepsilon}\right\}_{\varepsilon \in I}\right)>0$ such that

$$
\left\|\Lambda\left(F_{\varepsilon}\right)\right\|_{p, s} \leqslant c\left\|\left(x^{2}-\triangle\right)^{s / 2} \Lambda\right\|_{L_{p^{\prime}}(\mathbb{R}, \mathrm{d} x)}
$$

for every $\varepsilon \in I$ and $\Lambda \in \mathscr{S}^{\prime}(\mathbb{R})$ with $\left(x^{2}-\triangle\right)^{s / 2} \Lambda \in L_{p^{\prime}}(\mathbb{R}, \mathrm{d} x)$.

See also Remark A.5. The following proof is based on the technique in Watanabe (1991).

Proof: We show in the case of $-2 \leqslant s \leqslant 1$. Other cases are similarly proved. Define a linear operator $T_{\alpha}(\varepsilon)$ for $-2 \leqslant \alpha \leqslant 1$ and $\varepsilon \in I$ by

$$
T_{\alpha}(\varepsilon) \phi:=(I-\mathcal{L})^{\alpha / 2}\left[\left(x^{2}-\triangle\right)^{-\alpha / 2} \phi\right]\left(F_{\varepsilon}\right)
$$

for $\phi=\phi(x) \in \mathscr{S}(\mathbb{R})$. Since $\left\{F_{\varepsilon}\right\}_{\varepsilon \in I}$ is uniformly non-degenerate, the density function $p_{F_{\varepsilon}}(x)$ is uniformly bounded in $(\varepsilon, x) \in I \times \mathbb{R}$ :

$$
c_{0}:=\sup _{\varepsilon \in I} \sup _{x \in \mathbb{R}} p_{F_{\varepsilon}}(x)<\infty .
$$

Take $p^{\prime}>p>1$ arbitrary. We divide the proof into five steps.

(a) In the first place, when $\alpha=-2$, we shall show that $T_{-2}(\varepsilon): L_{p^{\prime}}(\mathbb{R}, \mathrm{d} x) \rightarrow$ $\mathbb{D}_{p}^{0}=L_{p}$ and is a continuous linear operator with an estimate

$$
\left\|T_{-2}(\varepsilon)\right\|_{L_{p^{\prime}}(\mathbb{R}, \mathrm{d} x) \rightarrow L_{p}} \leqslant c_{1} \quad \text { for any } \varepsilon \in I
$$

for some constant $c_{1}>0$. Let $\phi \in \mathscr{S}(\mathbb{R})$ be arbitrary. Then

$$
\left\|T_{-2}(\varepsilon) \phi\right\|_{p}=\left\|(I-\mathcal{L})^{-1}\left[\left(x^{2}-\triangle\right) \phi\right]\left(F_{\varepsilon}\right)\right\|_{p}=\left\|(H \phi)\left(F_{\varepsilon}\right)\right\|_{p,-2},
$$

where $H:=x^{2}-\triangle$, and for each $J \in \mathbb{D}^{\infty}$ and $p^{\prime \prime} \in\left(p, p^{\prime}\right)$, we have

$$
\mathbf{E}\left[(H \phi)\left(F_{\varepsilon}\right) J\right]=\mathbf{E}\left[\left(\left(x^{2}-\triangle\right) \phi\right)\left(F_{\varepsilon}\right) J\right] \leqslant\left\|\phi\left(F_{\varepsilon}\right)\right\|_{p^{\prime \prime}}\left\|l_{\varepsilon}(J)\right\|_{q^{\prime \prime}},
$$

where $1 / p^{\prime \prime}+1 / q^{\prime \prime}=1$ and $l_{\varepsilon}(J)$ is of the form $l_{\varepsilon}(J)=\sum_{i=0}^{2}\left\langle P_{i}(\varepsilon, w), D^{i} J\right\rangle_{H^{\otimes i}}$ for some $P_{i}(\varepsilon, w) \in \mathbb{D}^{\infty}\left(H^{\otimes i}\right), i=0,1,2$, all of which are polynomials in $F_{\varepsilon}$, its derivatives up to the second order and $\left\|D F_{\varepsilon}\right\|_{H}^{-2}$. Since $\left\{F_{\varepsilon}\right\}_{\varepsilon \in I}$ is uniformly nondegenerate, there exists $c_{0}^{\prime}>0$ such that $\left\|l_{\varepsilon}(J)\right\|_{q^{\prime \prime}} \leqslant c_{0}^{\prime}\|J\|_{q, 2}$. Hence we have

$$
\mathbf{E}\left[(H \phi)\left(F_{\varepsilon}\right) J\right] \leqslant c_{0}^{\prime}\left\|\phi\left(F_{\varepsilon}\right)\right\|_{p^{\prime \prime}}\|J\|_{q, 2} \leqslant c_{0} c_{0}^{\prime}\|\phi\|_{L_{p^{\prime}}(\mathbb{R}, \mathrm{d} x)}\|J\|_{q, 2}
$$


for each $J \in \mathbb{D}^{\infty}$, which implies $\left\|T_{-2}(\varepsilon) \phi\right\|_{p} \leqslant c_{1}\|\phi\|_{L_{p^{\prime}}(\mathbb{R}, \mathrm{d} x)}$, where $c_{1}:=c_{0} c_{0}^{\prime}$. Since $\mathscr{S}(\mathbb{R})$ is dense in $L_{p^{\prime}}(\mathbb{R}, \mathrm{d} x)$, we obtain the desired estimate.

(b) Next, we focus on the case of $\alpha=1$. We shall show that this operator actually defines a continuous linear mapping $T_{1}(\varepsilon): L_{p^{\prime}}(\mathbb{R}, \mathrm{d} x) \rightarrow \mathbb{D}_{p}^{0}=L_{p}$ with an estimate

$$
\left\|T_{1}(\varepsilon)\right\|_{L_{p^{\prime}}(\mathbb{R}, \mathrm{d} x) \rightarrow L_{p}} \leqslant c_{2} \quad \text { for any } \varepsilon \in I,
$$

for some constant $c_{2}>0$. Let $\phi=\phi(x) \in \mathscr{S}(\mathbb{R})$ be arbitrary. Then

$$
\left\|T_{1}(\varepsilon) \phi\right\|_{L_{p}}=\left\|(I-\mathcal{L})^{1 / 2}\left[\left(x^{2}-\triangle\right)^{-1 / 2} \phi\right]\left(F_{\varepsilon}\right)\right\|_{L_{p}}=\left\|\left(H^{-1 / 2} \phi\right)\left(F_{\varepsilon}\right)\right\|_{p, 1} .
$$

By Meyer's inequality, there exists a positive constant $c_{2}^{\prime}>0$ such that $\|J\|_{p, 1} \leqslant$ $c_{2}^{\prime}\left(\|J\|_{L_{p}}+\|D J\|_{L_{p}(H)}\right)$ for every $J \in \mathbb{D}_{2}^{1}$. Hence we have

$$
\left\|\left(H^{-1 / 2} \phi\right)\left(F_{\varepsilon}\right)\right\|_{p, 1} \leqslant c_{2}^{\prime}\left(\left\|\left(H^{-1 / 2} \phi\right)\left(F_{\varepsilon}\right)\right\|_{L_{p}}+\left\|\left(H^{-1 / 2} \phi\right)^{\prime}\left(F_{\varepsilon}\right) D F_{\varepsilon}\right\|_{L_{p}(H)}\right) .
$$

We easily have $\left\|\left(H^{-1 / 2} \phi\right)\left(F_{\varepsilon}\right)\right\|_{L_{p}} \leqslant c_{0}\left\|\left(x^{2}-\triangle\right)^{-1 / 2} \phi\right\|_{L_{p^{\prime}}(\mathbb{R}, \mathrm{d} x)}$. Take $q^{\prime} \in(1, \infty)$ so that $1 / p^{\prime}+1 / q^{\prime}=1 / p$. Then there exists $c_{2}^{\prime \prime}=c_{2}^{\prime \prime}\left(p^{\prime}, q^{\prime}\right)>0$ such that $\left\|J_{1} J_{2}\right\|_{L_{p}(H)} \leqslant c_{2}^{\prime \prime}\left\|J_{1}\right\|_{L_{p^{\prime}}}\left\|J_{2}\right\|_{L_{q^{\prime}}(H)}$ for all $\left(J_{1}, J_{2}\right) \in L_{p^{\prime}} \times L_{q^{\prime}}(H)$. Hence we have

$$
\begin{aligned}
& \left\|\left(H^{-1 / 2} \phi\right)^{\prime}\left(F_{\varepsilon}\right) D F_{\varepsilon}\right\|_{L_{p}(H)} \\
& \leqslant c_{2}^{\prime \prime}\left\|\left[\left(x^{2}-\triangle\right)^{-1 / 2} \phi\right]^{\prime}\left(F_{\varepsilon}\right)\right\|_{L_{p^{\prime}}}\left\|D F_{\varepsilon}\right\|_{L_{q^{\prime}}(H)} \\
& \leqslant c_{2}^{\prime \prime} c_{0}\left(\sup _{\varepsilon \in I}\left\|D F_{\varepsilon}\right\|_{L_{q^{\prime}}(H)}\right)\left\|\left[\left(x^{2}-\triangle\right)^{-1 / 2} \phi\right]^{\prime}\right\|_{L_{p^{\prime}}(\mathbb{R}, \mathrm{d} x)} .
\end{aligned}
$$

By a result by Bongioanni and Torrea (2006, Lemma 3 and Theorem 4), there exists a constant $c_{2}^{\prime \prime \prime}=c_{2}^{\prime \prime \prime}\left(p^{\prime}\right)>0$ such that

$$
\begin{aligned}
& \left\|\left[\left(x^{2}-\triangle\right)^{-1 / 2} \phi\right]^{\prime}\right\|_{L_{p^{\prime}}(\mathbb{R}, \mathrm{d} x)}+\left\|\left(x^{2}-\triangle\right)^{-1 / 2} \phi\right\|_{L_{p^{\prime}}(\mathbb{R}, \mathrm{d} x)} \\
& \leqslant\left\|\left(\frac{\mathrm{d}}{\mathrm{d} x}+x\right)\left(x^{2}-\triangle\right)^{-1 / 2} \phi\right\|_{L_{p^{\prime}}(\mathbb{R}, \mathrm{d} x)}+\left\|\left(x^{2}-\triangle\right)^{-1 / 2} \phi\right\|_{L_{p^{\prime}}(\mathbb{R}, \mathrm{d} x)} \\
& \quad+\left\|x\left(x^{2}-\triangle\right)^{-1 / 2} \phi\right\|_{L_{p^{\prime}}(\mathbb{R}, \mathrm{d} x)} \\
& \leqslant c_{2}^{\prime \prime \prime}\|\phi\|_{L_{p^{\prime}}(\mathbb{R}, \mathrm{d} x)},
\end{aligned}
$$

and hence we have obtained

$$
\left\|T_{1}(\varepsilon) \phi\right\|_{L_{p}} \leqslant c_{2}\|\phi\|_{L_{p^{\prime}}(\mathbb{R}, \mathrm{d} x)} \quad \text { for all } \varepsilon \in I
$$

as desired, where $c_{2}:=c_{0} c_{2}^{\prime}\left(1+c_{2}^{\prime \prime} \sup _{\varepsilon \in I}\left\|D \widetilde{F}_{\varepsilon}\right\|_{L_{q^{\prime}}(H)}\right) c_{2}^{\prime \prime \prime}$.

(c) For each $\varepsilon \in I, z \in \mathbb{C}$ and $\phi \in \mathscr{S}(\mathbb{R})$, we define

$$
T_{z}(\varepsilon) \phi:=(I-\mathcal{L})^{z / 2}\left[\left(x^{2}-\triangle\right)^{-z / 2} \phi\right]\left(F_{\varepsilon}\right), \quad \phi \in \mathscr{S}(\mathbb{R})
$$

(the operators $(I-\mathcal{L})^{z / 2}$ and $\left(x^{2}-\triangle\right)^{-z / 2}$ are defined by the same definition formulae for the real-exponent case). For any $\phi \in \mathscr{S}(\mathbb{R})$ and $\psi \in \mathbb{D}^{\infty}$, we shall show $z \mapsto \mathbf{E}\left[\left(T_{z}(\varepsilon) \phi\right) \psi\right]$ is analytic. For this, we note that

$$
\begin{aligned}
\mathbf{E}\left[\left(T_{z}(\varepsilon) \phi\right) \psi\right] & =\mathbf{E}\left[\left(H^{-z / 2} \phi\right)\left(F_{\varepsilon}\right)(I-\mathcal{L})^{z / 2} \psi\right] \\
& =\sum_{m=0}^{\infty}(1+m)^{z / 2} \mathbf{E}\left[\left(H^{-z / 2} \phi\right)\left(F_{\varepsilon}\right) J_{m}[\psi]\right] .
\end{aligned}
$$


Letting $\left\{\phi_{n}\right\}_{n \in \mathbb{Z}_{\geqslant 0}}$ be as in Lemma A.1, we have $H^{-z / 2} \phi_{n}=(2 n+1)^{-z / 2} \phi_{n}$ for $n \in \mathbb{Z}_{\geqslant 0}$ and $\left\{\widetilde{\phi}_{n}:=(\sqrt{\pi} n !)^{-1 / 2} \phi_{n}\right\}_{n \in \mathbb{Z} \geqslant 0}$ is a complete orthonormal system of $L_{2}(\mathbb{R}, \mathrm{d} x)$. Then we have $\phi=\sum_{n=0}^{\infty}\left\langle\phi, \widetilde{\phi}_{n}\right\rangle_{L_{2}(\mathbb{R}, \mathrm{d} x)} \widetilde{\phi}_{n}$ in $L_{2}(\mathbb{R}, \mathrm{d} x)$. Therefore

$$
\mathbf{E}\left[\left(H^{-z / 2} \phi\right)\left(F_{\varepsilon}\right) J_{m}[\psi]\right]=\sum_{n=0}^{\infty}(2 n+1)^{-z / 2}\left\langle\phi, \widetilde{\phi}_{n}\right\rangle_{L_{2}(\mathbb{R}, \mathrm{d} x)} \mathbf{E}\left[\widetilde{\phi}_{n}\left(F_{\varepsilon}\right) J_{m}[\psi]\right]
$$

is an infinite sum of analytic functions in $z$. Furthermore, this series converges absolutely and locally-uniformly in $z$. In fact, with recalling that $c_{0}=$ $\sup _{\varepsilon \in I} \sup _{x \in \mathbb{R}} p_{F_{\varepsilon}}(x)<\infty$ where $p_{F_{\varepsilon}}$ is the probability density function of $F_{\varepsilon}$, and by using the Cauchy-Schwartz inequality,

$$
\begin{aligned}
& \sum_{n=0}^{\infty}\left|(2 n+1)^{-z / 2}\left\langle\phi, \widetilde{\phi}_{n}\right\rangle_{L_{2}(\mathbb{R}, \mathrm{d} x)} \mathbf{E}\left[\widetilde{\phi}_{n}\left(F_{\varepsilon}\right) J_{m}[\psi]\right]\right| \\
& \leqslant c_{0}\left\|J_{m}[\psi]\right\|_{L_{2}} \sum_{n=0}^{\infty}\left|(2 n+1)^{-1}\left\langle\phi, H^{1-\operatorname{Re}(z / 2)} \widetilde{\phi}_{n}\right\rangle_{L_{2}(\mathbb{R}, \mathrm{d} x)}\right| \\
& \leqslant c_{0}\left\|J_{m}[\psi]\right\|_{L_{2}}\left(\sum_{n=0}^{\infty}(2 n+1)^{-2}\right)^{1 / 2}\left\|H^{1-\operatorname{Re}(z / 2)} \phi\right\|_{L_{2}(\mathbb{R}, \mathrm{d} x)},
\end{aligned}
$$

is finite because $\phi \in \mathscr{S}(\mathbb{R})$. Therefore, $\mathbf{E}\left[\left(H^{-z / 2} \phi\right)\left(F_{\varepsilon}\right) J_{m}[\psi]\right]$ is indeed an analytic function in $z$. Thus each term in the summation in (A.3) is analytic in $z$. We see further that the each term has the estimate

$$
\begin{aligned}
& \left|\mathbf{E}\left[\left(H^{-z / 2} \phi\right)\left(F_{\varepsilon}\right) J_{m}[\psi]\right]\right| \\
& \leqslant\left\|\left(H^{-z / 2} \phi\right)\left(F_{\varepsilon}\right)\right\|_{L_{2}}\left\|J_{m}[\psi]\right\|_{L_{2}} \\
& \leqslant c_{0}\left\|H^{-z / 2} \phi\right\|_{L_{2}(\mathbb{R}, \mathrm{d} y)}\left\|J_{m}[\psi]\right\|_{L_{2}} .
\end{aligned}
$$

Therefore

$$
\sum_{m=0}^{\infty}\left|(1+m)^{z / 2} \mathbf{E}\left[\left(H^{-z / 2} \phi\right)\left(F_{\varepsilon}\right) J_{m}[\psi]\right]\right| \leqslant c_{0}\left\|H^{-z / 2} \phi\right\|_{L_{2}(\mathbb{R}, \mathrm{d} y)}\|\psi\|_{2, \operatorname{Re}(z)} .
$$

Here, the two quantities $\left\|H^{-z / 2} \phi\right\|_{L_{2}(\mathbb{R}, \mathrm{d} y)}$ and $\|\psi\|_{2, \operatorname{Re}(z)}$ are continuously depending on $z$ because $\phi \in \mathscr{S}(\mathbb{R})$ and $\psi \in \mathbb{D}^{\infty}$. Hence the infinite sum in (A.3) converges absolutely and locally-uniformly in $z$, so that $\mathbf{E}\left[\left(T_{z}(\varepsilon) \phi\right) \psi\right]$ is analytic in $z$.

(d) For $\phi \in \mathscr{S}(\mathbb{R})$ and $\psi \in \mathbb{D}^{\infty}, \Phi(z):=\mathbf{E}\left[\left(T_{z}(\varepsilon) \phi\right) \psi\right]$ is analytic on $\mathbb{C}$. By the Marcinkiewicz $L_{p}$-multiplier theorem (see e.g. Thangavelu, 1993, Chapter 4, Section 4.2, Theorem 4.2.1), one gets $\sup _{\tau \in \mathbb{R}}\left\|\left(x^{2}-\triangle\right)^{i \tau}\right\|_{L_{p^{\prime}}(\mathbb{R}, \mathrm{d} x) \rightarrow L_{p^{\prime}}(\mathbb{R} \rightarrow \mathbb{C} ; \mathrm{d} x)}<+\infty$, where $L_{p^{\prime}}(\mathbb{R} \rightarrow \mathbb{C} ; \mathrm{d} x)$ is the space of all complex-valued measurable functions that are $p^{\prime}$-th order integrable. On the other hand, by Meyer's $L_{p}$-multiplier theorem (see e.g. Ikeda and Watanabe, 1989, Chapter V, Section 8, Lemma 8.2) gives $\sup _{\tau \in \mathbb{R}}\left\|(I-\mathcal{L})^{i \tau}\right\|_{L_{p} \rightarrow \mathbb{D}_{p}^{0}(\mathbb{C})}<+\infty$. By using these, we can conclude the following:

(1) the complex function $\Phi$ has the estimate

$$
\sup _{\tau \in \mathbb{R}} \sup _{-2 \leqslant \alpha \leqslant 1}|\Phi(\alpha+i \tau)|<+\infty
$$

for each $\phi$ and $\psi$ (see Hirschman's Lemma in Stein, 1956). 
(2) for $\tau \in \mathbb{R}$, the operators $T_{-2+i \tau}(\varepsilon)$ and $T_{1+i \tau}(\varepsilon)$ uniquely extends to a bounded linear operators $L_{p^{\prime}}(\mathbb{R}, \mathrm{d} x) \rightarrow \mathbb{D}_{p}^{0}(\mathbb{C})$ with estimates

$$
\begin{aligned}
& \sup _{\tau \in \mathbb{R}}\left\|T_{-2+i \tau}(\varepsilon)\right\|_{L_{p^{\prime}}(\mathbb{R}, \mathrm{d} x) \rightarrow \mathbb{D}_{p}^{0}(\mathbb{C})}<+\infty, \\
& \sup _{\tau \in \mathbb{R}}\left\|T_{1+i \tau}(\varepsilon)\right\|_{L_{p^{\prime}}(\mathbb{R}, \mathrm{d} x) \rightarrow \mathbb{D}_{p}^{0}(\mathbb{C})}<+\infty
\end{aligned}
$$

(Note that one can take these upper bounds independent of $\varepsilon$ since the bounds $c_{1}$ and $c_{2}$ obtained in steps (a) and (b) are independent of $\varepsilon$ ). Then by Stein's interpolation theorem ${ }^{2}$ (see Stein, 1956, Theorem 1), we can conclude that the operators $T_{z}(\varepsilon)$ for $-2 \leqslant \operatorname{Re}(z) \leqslant 1$ uniquely extends to a bounded linear operator $L_{p^{\prime}}(\mathbb{R}, \mathrm{d} x) \rightarrow \mathbb{D}_{p}^{0}(\mathbb{C})$ and

$$
\sup _{\varepsilon \in I-2 \leqslant \alpha \leqslant 1} \sup _{-2 \leqslant}\left\|T_{\alpha}(\varepsilon)\right\|_{L_{p^{\prime}}(\mathbb{R}, \mathrm{d} x) \rightarrow L_{p}}<+\infty .
$$

(e) Now, for each $\phi \in \mathscr{S}(\mathbb{R})$, we have

$$
\begin{aligned}
& \left\|\phi\left(F_{\varepsilon}\right)\right\|_{p, s}=\left\|(I-\mathcal{L})^{s / 2}\left[\left(x^{2}-\triangle\right)^{-s / 2}\left(x^{2}-\triangle\right)^{s / 2} \phi\right]\left(F_{\varepsilon}\right)\right\|_{L_{p}} \\
& =\left\|T_{s}(\varepsilon)\left[\left(x^{2}-\triangle\right)^{s / 2} \phi\right]\right\|_{L_{p}} \\
& \leqslant\left\|T_{s}(\varepsilon)\right\|_{L_{p^{\prime}}(\mathbb{R}, \mathrm{d} x) \rightarrow L_{p}}\left\|\left[\left(x^{2}-\triangle\right)^{s / 2} \phi\right]\right\|_{L_{p^{\prime}}(\mathbb{R}, \mathrm{d} x)} .
\end{aligned}
$$

Again by denseness of $\mathscr{S}(\mathbb{R})$, this inequality extends to $\Lambda \in \mathscr{S}^{\prime}(\mathbb{R})$ such that $\left(x^{2}-\triangle\right)^{s / 2} \Lambda \in L_{p^{\prime}}(\mathbb{R}, \mathrm{d} x)$.

Remark A.5. In Lemma A.4 and Lemma 4.12, one can take $p^{\prime}=p$ when $F_{\varepsilon}=$ $\varepsilon^{-1} w\left(\varepsilon^{2} T\right)$, where $\varepsilon \in(0, T]=: I, T>0$ and $w=\left(w^{1}, \cdots, w^{d}\right)$ is the canonical process, i.e., the $d$-dimensional Wiener process starting at zero (assume $d=1$ if considering Lemma A.4), because then $D F_{\varepsilon}=\varepsilon^{-1} D w\left(\varepsilon^{2} T\right)=\varepsilon^{-1}\left(1_{\left[0, \varepsilon^{2} T\right]}, \cdots, 1_{\left[0, \varepsilon^{2} T\right]}\right)$ and $\left\langle D F_{\varepsilon}^{i}, D F_{\varepsilon}^{j}\right\rangle_{H}=\delta_{i j} T$ are non-random.

A.4. Regularity of something related to resolvent kernel associated with elliptic operators. Suppose (H1), (H3) and (H4). We set, for $f \in \mathscr{S}\left(\mathbb{R}^{d}\right)$ and $x \in \mathbb{R}^{d}$,

$$
L f(x)=\frac{1}{2} \sum_{i, j=1}^{d}\left(\sigma \sigma^{*}\right)_{j}^{i}(x) \frac{\partial^{2} f}{\partial x_{i} \partial x_{j}}(x)+\sum_{i=1}^{d} b^{i}(x) \frac{\partial f}{\partial x_{i}}(x) .
$$

The fractional power $(1-\triangle)^{s / 2}$ is defined as a pseudo-differential operator:

$$
(1-\triangle)^{s / 2} \phi(x)=\int_{\mathbb{R}^{d}}\left(1+|\xi|^{2}\right)^{s / 2} \widehat{\phi}(\xi) \mathrm{e}^{i\langle\xi, x\rangle} \mathrm{d} \xi, \quad \phi \in \mathscr{S}\left(\mathbb{R}^{d}\right),
$$

where $\widehat{\phi}(\xi)=(2 \pi)^{-d / 2} \int_{\mathbb{R}^{d}} \phi(y) \mathrm{e}^{-i\langle\xi, y\rangle} \mathrm{d} y$ is the Fourier transform of $\phi$, and is also given by

$$
(1-\triangle)^{s / 2} \phi(x)=\frac{1}{\Gamma(-s / 2)} \int_{0}^{\infty} t^{-\frac{s}{2}-1} \mathrm{e}^{-t}\left(\mathrm{e}^{t \triangle} \phi\right)(x) \mathrm{d} t, \quad \phi \in \mathscr{S}\left(\mathbb{R}^{d}\right)
$$

\footnotetext{
${ }^{2}$ Strictly speaking, this theorem was stated in the case where $\phi$ and $\psi$ are simple functions in Stein (1956, Theorem 1). This is because just that his argument is based on general $\sigma$-finite measure spaces where we can not speak about 'smoothness'. The same arguments apply to our case.
} 
for $-d<s<0$, where $\mathrm{e}^{t \triangle}$ is the heat semigroup associated to $\triangle$. Actually, formulae $\phi(y)=\int_{\mathbb{R}^{d}} \widehat{\phi}(\xi) \mathrm{e}^{i\langle\xi, y\rangle} \mathrm{d} \xi$ and $\int_{\mathbb{R}^{d}} \mathrm{e}^{-\frac{|y|^{2}}{4 t}} \mathrm{e}^{i\langle\xi, y\rangle} \mathrm{d} y=(4 \pi t)^{d / 2} \mathrm{e}^{-|\xi|^{2} t}$ give the above equivalence.

Before entering the following series of estimates, we recall that

$$
\int_{|x|<1}|x|^{-s} \mathrm{~d} x<+\infty \quad \text { iff } \quad s<d
$$

where the integral is over $\mathbb{R}^{d}$ and $|x|=|x|_{\mathbb{R}^{d}}$.

Lemma A.6. For every $p \in(1, \infty)$ and $s \in(-d,-(p-1) d / p)$, we have $(1-$ $\triangle)^{s / 2} \delta_{y} \in L_{p}\left(\mathbb{R}^{d}, \mathrm{~d} x\right)$ for each $y \in \mathbb{R}^{d}$.

Proof: The transition density associated to the semigroup generated by $\triangle$ is given by $p_{t}(x, y)=(4 \pi t)^{-d / 2} \exp \left(-|y-x|^{2} /(4 t)\right)$. Hence we have

$$
\begin{aligned}
& (1-\triangle)^{s / 2} \delta_{y}(x)=\frac{1}{\Gamma(-s / 2)} \int_{0}^{\infty} t^{-\frac{s}{2}-1} \mathrm{e}^{-t} p_{t}(x, y) \mathrm{d} t \\
& =\frac{(4 \pi)^{-d / 2}}{\Gamma(-s / 2)} \int_{0}^{\infty} t^{-\frac{s+d}{2}-1} \mathrm{e}^{-t} \exp \left\{-\frac{|x-y|^{2}}{4 t}\right\} \mathrm{d} t \\
& =\frac{(4 \pi)^{-d / 2}}{4^{-\frac{s+d}{2}} \Gamma(-s / 2)}|x-y|^{-(d+s)} \int_{0}^{\infty} u^{\frac{d+s}{2}-1} \mathrm{e}^{-u} \exp \left\{-\frac{|x-y|^{2}}{4 u}\right\} \mathrm{d} u,
\end{aligned}
$$

which behaves, up to a multiplicative constant, as $|x-y|^{-(d+s)}$ when $|x-y| \rightarrow 0$ and rapidly decreasing as $|x-y| \rightarrow \infty$. In fact, by using the identity

$$
\mathrm{e}^{-u} \exp \left\{-\frac{|x-y|^{2}}{4 u}\right\}=\exp \left\{-\frac{(|x-y|-2 u)^{2}}{4 u}\right\} \mathrm{e}^{-|x-y|}
$$

and putting $a:=|x-y|$, the last integral can be written as

$$
\int_{0}^{\infty} u^{\frac{d+s}{2}-1} \mathrm{e}^{-u} \exp \left\{-\frac{a^{2}}{4 u}\right\} \mathrm{d} u=\mathrm{e}^{-a}(I+I I)
$$

where

$$
\begin{aligned}
I & :=\int_{0}^{a} u^{\frac{d+s}{2}-1} \exp \left\{-\frac{(a-2 u)^{2}}{4 u}\right\} \mathrm{d} u, \\
I I & :=\int_{a}^{\infty} u^{\frac{d+s}{2}-1} \exp \left\{-\frac{(a-2 u)^{2}}{4 u}\right\} \mathrm{d} u .
\end{aligned}
$$

Since $d+s>0$, we have $I \leqslant \int_{0}^{a} u^{\frac{d+s}{2}-1} \mathrm{~d} u=\frac{2}{d+s} a^{\frac{d+s}{2}}$. On the other hand, by using the change of variable $u=\frac{a}{2} v$, one has $I I=(a / 2)^{\frac{d+s}{2}} \int_{2}^{+\infty} v^{\frac{d+s}{2}-1} \exp \left(-\frac{(1-v)^{2}}{2 v} a\right) \mathrm{d} v$. For $a \geqslant 1$ and $v \geqslant 2$, we have $\exp \left(-\frac{(1-v)^{2}}{2 v} a\right)=\exp \left(-\frac{a}{2}(v-2)\right) \exp \left(-\frac{a}{2 v}\right) \leqslant$ $\exp \left(-\frac{a}{2}(v-2)\right) \leqslant \exp \left(-\frac{1}{2}(v-2)\right)=\mathrm{e} \cdot \exp (-v / 2)$. Thus we obtain $I I \leqslant c_{0} \mathrm{e}(a / 2)^{\frac{d+s}{2}}$ if $a \geqslant 1$, where $c_{0}:=\int_{2}^{+\infty} v^{\frac{d+s}{2}-1} \exp (-v / 2) \mathrm{d} v<+\infty$. The arguments above shows that $\int_{0}^{\infty} u^{\frac{d+s}{2}-1} \mathrm{e}^{-u} \exp \left(-\frac{a^{2}}{4 u}\right) \mathrm{d} u$ decreases rapidly when $a \rightarrow+\infty$, as claimed.

Therefore, $(1-\triangle)^{s / 2} \delta_{y} \in L_{p}\left(\mathbb{R}^{d}, \mathrm{~d} x\right)$ if $(d+s) p<d$, i.e., $s<-(p-1) d / p$.

Let $x \in \mathbb{R}^{d}$. Under the conditions (H1), (H3) and (H4), we denote by $X_{t}=$ $X(t, x, w)$ a unique strong solution to the stochastic differential equation (4.10). In the sequel, we fix $y \in \mathbb{R}^{d}$ such that $y \neq x$ and define

$$
f_{y}(z):=(1-L)^{-1} \delta_{y}(z), \quad z \in \mathbb{R}^{d} .
$$


Let $\phi: \mathbb{R}^{d} \rightarrow \mathbb{R}$ be a $C^{\infty}$-function such that (1) $x \notin \operatorname{supp} \phi,(2) \phi \equiv 1$ on a neighbourhood of $y$, and (3) $\operatorname{supp} \phi$ is compact.

Lemma A.7. Assume (H1), (H3) and (H4). Let $p \in(1, \infty)$ be arbitrary.

(i) For each $s<\min \left\{1-\frac{(p-1) d}{p}, 0\right\}$ and $t>0$, we have $\left(\nabla f_{y}\right)\left(X_{t}\right) \in \mathbb{D}_{p}^{s}\left(\mathbb{R}^{d}\right)$ and $(0, T] \ni t \mapsto\left(\nabla f_{y}\right)\left(X_{t}\right) \in \mathbb{D}_{p}^{s}\left(\mathbb{R}^{d}\right)$ is continuous.

(ii) Assume $d \geqslant 2$. Then for each $-\frac{d}{2}<s<\min \left\{\frac{p}{p-1}-d, 0\right\}$, we have $\lim _{t \rightarrow 0}\left\|\left(\nabla\left(\phi f_{y}\right)\right)\left(X_{t}\right)\right\|_{p, s}=0$ and $\lim _{t \rightarrow 0}\left\|\left(\phi f_{y}\right)\left(X_{t}\right)\right\|_{p, s}=0$.

Proof: Let $p \in(1, \infty)$ and $t_{0}>0$ be arbitrary. In the following, we write $f:=f_{y}$.

(i) Take $p^{\prime}>p$ such that $s<\min \left\{1-\frac{\left(p^{\prime}-1\right) d}{p^{\prime}}, 0\right\}$. Since $\left\{X_{t}\right\}_{t_{0} \leqslant t \leqslant T}$ is uniformly non-degenerate, the assertion follows by Lemma 4.12 once we show (1$\triangle)^{s / 2}\left(\partial f / \partial z_{k}\right) \in L_{p^{\prime}}\left(\mathbb{R}^{d}, \mathrm{~d} z\right)$.

Denote by $p_{t}\left(z, z^{\prime}\right)$ the transition density associated to $L$. By a standard estimate (see e.g., Friedman, 1964, Chapter 9, Section 6, Theorem 7), there exist $c, C>0$ such that

$$
\begin{gathered}
p_{t}\left(z, z^{\prime}\right) \leqslant C(2 \pi c t)^{-d / 2} \exp \left\{-\frac{\left|z-z^{\prime}\right|^{2}}{2 c t}\right\} \\
\left|\frac{\partial p_{t}}{\partial z_{k}}\left(z, z^{\prime}\right)\right| \leqslant C t^{-1 / 2}(2 \pi c t)^{-d / 2} \exp \left\{-\frac{\left|z-z^{\prime}\right|^{2}}{2 c t}\right\}
\end{gathered}
$$

for every $k=1,2, \cdots, d$ and $z, z^{\prime} \in \mathbb{R}^{d}$. We may assume $c \geqslant 2$ by rearranging $C>0$. Then we have

$$
\begin{aligned}
\left|\frac{\partial f}{\partial z_{k}}(z)\right| & \leqslant \frac{1}{\Gamma(1)} \int_{0}^{\infty} \mathrm{e}^{-t}\left|\frac{\partial p_{t}}{\partial z_{k}}(z, y)\right| \mathrm{d} t \\
& \leqslant C \int_{0}^{\infty} t^{-1 / 2} \mathrm{e}^{-t}(2 \pi c t)^{-d / 2} \exp \left\{-\frac{|z-y|^{2}}{2 c t}\right\} \mathrm{d} t
\end{aligned}
$$

so that

$$
\begin{aligned}
& \left|\left[(1-\triangle)^{s / 2} \frac{\partial f}{\partial z_{k}}\right](z)\right| \\
& \leqslant \frac{1}{\Gamma(-s / 2)} \int_{0}^{\infty} t^{-\frac{s}{2}-1} \mathrm{e}^{-t} \int_{\mathbb{R}^{d}} \frac{\mathrm{e}^{-\frac{\left|z-z^{\prime}\right|^{2}}{4 t}}}{\sqrt{4 \pi t}^{d}}\left|\frac{\partial f}{\partial z_{k}}\left(z^{\prime}\right)\right| \mathrm{d} z^{\prime} \mathrm{d} t \\
& \leqslant \frac{C^{\prime}}{\Gamma(-s / 2) \Gamma(1 / 2)} \int_{0}^{\infty} \int_{0}^{\infty} t^{-\frac{s}{2}-1} u^{-1 / 2} \mathrm{e}^{-(t+u)} \frac{\mathrm{e}^{-\frac{1 z-\left.y\right|^{2}}{2 c(t+u)}}}{(2 \pi c(t+u))^{d / 2}} \mathrm{~d} u \mathrm{~d} t \\
& =\frac{C^{\prime}}{\Gamma\left(\frac{1-s}{2}\right)} \int_{0}^{\infty} v^{\frac{1-s}{2}-1} \mathrm{e}^{-v} \frac{\mathrm{e}^{-\frac{|z-y|^{2}}{2 c v}}}{(2 \pi c v)^{d / 2}} \mathrm{~d} v,
\end{aligned}
$$

for some constant $C^{\prime}>0$. Hence $(1-\triangle)^{s / 2}\left(\partial_{k} f\right)(z) \in L_{p^{\prime}}\left(\mathbb{R}^{d}, \mathrm{~d} z\right)$.

(ii) Suppose that $s<\min \left\{\frac{p}{p-1}-d, 0\right\}$. Take $\delta>0$ so that $\left\{z \in \mathbb{R}^{d}:|z-x|<\delta\right\} \subset$ $(\operatorname{supp} \phi)^{c}$. Note that $\partial_{k}\left(\phi f_{y}\right)=\left(\partial_{k} \phi\right) f_{y}+\phi\left(\partial_{k} f_{y}\right),\left(\partial_{k} \phi\right)(x) f_{y}(x)=0$ and $\left(\partial_{k} \phi\right) f_{y} \in$ $\mathscr{S}\left(\mathbb{R}^{d}\right)$. Therefore by bounded convergence theorem, $\lim _{t \downarrow 0}\left\|\left[\left(\partial_{k} \phi\right) f_{y}\right]\left(X_{t}\right)\right\|_{p, s} \leqslant$ $\lim _{t \downarrow 0}\left\|\left[\left(\partial_{k} \phi\right) f_{y}\right]\left(X_{t}\right)\right\|_{p}=0$. So in the following, we investigate the behaviour of $\left(\phi\left(\partial_{k} f_{y}\right)\right)\left(X_{t}\right)$ and $\left(\phi f_{y}\right)\left(X_{t}\right)$. For this, we divide the proof into four steps. 
(a) Since $s \leqslant 0$, we notice that

$$
\begin{aligned}
\left|(I-\mathcal{L})^{s / 2} F\right| & =\left|\frac{1}{\Gamma\left(-\frac{s}{2}\right)} \int_{0}^{\infty} u^{-\frac{s}{2}-1} \mathrm{e}^{-u} T_{u} F \mathrm{~d} u\right| \\
& \leqslant \frac{1}{\Gamma\left(-\frac{s}{2}\right)} \int_{0}^{\infty} u^{-\frac{s}{2}-1} \mathrm{e}^{-u} T_{u}|F| \mathrm{d} u=(I-\mathcal{L})^{s / 2}|F|
\end{aligned}
$$

for any $F \in L_{2}$, where $T_{u}=\exp (u \mathcal{L}), u \geqslant 0$ is the Ornstein-Uhlenbeck semigroup on the Wiener space. Then, taking $p^{\prime}, q, r>1$ so that $\frac{1}{p^{\prime}}+\frac{1}{q}+\frac{1}{r}<1$ and with putting $F:=\left(\phi \partial_{k} f\right)\left(X_{t}\right)=\left(\phi \partial_{k} f\right)\left(X_{t}\right) 1_{\left\{\left|X_{t}-x\right|>\delta\right\}}$, we have

$$
\begin{aligned}
& \left\|\left(\phi \partial_{k} f\right)\left(X_{t}\right)\right\|_{p, s}^{p}=\left\|(I-\mathcal{L})^{s / 2} F\right\|_{p}^{p} \\
& \leqslant \mathbf{E}\left[\left|(I-\mathcal{L})^{s / 2} F\right|^{p-1}(I-\mathcal{L})^{s / 2}|F|\right] \\
& =\mathbf{E}\left[\left|\left(\phi \partial_{k} f\right)\left(X_{t}\right)\right|\left\{(I-\mathcal{L})^{s / 2}\left|(I-\mathcal{L})^{s / 2} F\right|^{p-1}\right\} 1_{\left\{\left|X_{t}-x\right|>\delta\right\}}\right] \\
& \leqslant c_{0}\left\|\left(\phi \partial_{k} f\right)\left(X_{t}\right)\right\|_{p^{\prime}}\left\|\left|(I-\mathcal{L})^{s / 2} F\right|^{p-1}\right\|_{q, s} \mathbf{P}\left(\left|X_{t}-x\right|>\delta\right)^{1 / r},
\end{aligned}
$$

where $c_{0}=c_{0}\left(p^{\prime}, q, r\right)>0$ is a constant independent of $t$. We easily have $\left\|\left(\phi \partial_{k} f\right)\left(X_{t}\right)\right\|_{p^{\prime}} \leqslant|\phi|_{\infty}\left\|\left(\partial_{k} f\right)\left(X_{t}\right)\right\|_{p^{\prime}}$ and

$$
\begin{aligned}
& \left.\|\|(I-\mathcal{L})^{s / 2} F\right|^{p-1} \|_{q, s} \\
& \leqslant\left\|\left\{(I-\mathcal{L})^{s / 2} F\right\}^{p-1}\right\|_{q} \\
& =\left\|(I-\mathcal{L})^{s / 2} F\right\|_{q(p-1)}^{p-1}=\left\|(I-\mathcal{L})^{s / 2}\left(\phi \partial_{k} f\right)\left(X_{t}\right)\right\|_{p^{\prime \prime}}^{p-1},
\end{aligned}
$$

where $p^{\prime \prime}:=q(p-1)$. Thus we have obtained

$$
\begin{aligned}
& \left\|\left(\phi \partial_{k} f\right)\left(X_{t}\right)\right\|_{p, s}^{p} \\
& \leqslant c_{0}|\phi|_{\infty}\left\|\left(\partial_{k} f\right)\left(X_{t}\right)\right\|_{p^{\prime}}\left\|(I-\mathcal{L})^{s / 2}\left(\phi \partial_{k} f\right)\left(X_{t}\right)\right\|_{p^{\prime \prime}}^{p-1} \\
& \times \mathbf{P}\left(\left|X_{t}-x\right|>\delta\right)^{1 / r}
\end{aligned}
$$

Similarly, we have

$$
\begin{aligned}
& \left\|(\phi f)\left(X_{t}\right)\right\|_{p, s}^{p} \\
& \leqslant c_{0}^{\prime}\left\|f\left(X_{t}\right)\right\|_{p^{\prime}}\left\|(I-\mathcal{L})^{s / 2}(\phi f)\left(X_{t}\right)\right\|_{p^{\prime \prime}}^{p-1} \\
& \quad \times \mathbf{P}\left(\left|X_{t}-x\right|>\delta\right)^{1 / r}
\end{aligned}
$$

for some constant $c_{0}^{\prime}>0$, independent of $t$.

(b) We will write $\varepsilon:=\sqrt{t}$ in the sequel. We shall give estimates for each factors in (A.5) and (A.6), though the proof is presented in the next step. Note that for the last factors in (A.5) and (A.6), there exists $c_{3}, c_{3}^{\prime}, K>0$ such that

$$
\mathbf{P}\left(\left|X_{t}-x\right|>\delta\right) \leqslant c_{3} \exp \left(-\frac{\delta^{2}}{c_{3}^{\prime} \varepsilon^{2}}\right) \quad \text { for } t=\varepsilon^{2} \in(0, K] .
$$

Let $p \in(1, \infty)$ anew be arbitrary. To see (A.5), we shall prove

$$
\begin{gathered}
\lim _{\varepsilon \downarrow 0} \varepsilon^{d}\left\|\left(\partial_{k} f\right)\left(X_{t}\right)\right\|_{p}^{p}=0 \quad \text { if } p<\frac{d}{d-1} \\
\lim _{\varepsilon \downarrow 0} \varepsilon^{d-s p}\left\|(I-\mathcal{L})^{s / 2}\left(\phi \partial_{k} f\right)\left(X_{t}\right)\right\|_{p}^{p}=0 \quad \text { if } p<\frac{d}{d+s-1} .
\end{gathered}
$$


On the other hand, for (A.6), we shall prove

$$
\begin{gathered}
\lim _{\varepsilon \downarrow 0} \varepsilon^{d}\left\|f\left(X_{t}\right)\right\|_{p}^{p}=0 \quad \text { if } p<\frac{d}{d-1}, \\
\lim _{\varepsilon \downarrow 0} \varepsilon^{d-s p}\left\|(I-\mathcal{L})^{s / 2}(\phi f)\left(X_{t}\right)\right\|_{p}^{p}=0 \\
\text { if } p<\min \left\{\frac{d}{d+s-1}, \frac{2 d}{d-2}\right\}=\frac{d}{d+s-1},
\end{gathered}
$$

where $\frac{2 d}{d-2}$ is understood as $+\infty$ if $d=2$ and the last equality follows from $s>-\frac{d}{2}$.

(c) Take $p^{\prime}>p$ arbitrary. First we shall prove (A.8). By using Proposition 3.7 and Lemma 4.12 (Recall that $\varepsilon=\sqrt{t}$ and $X_{t}=x+\varepsilon F_{\varepsilon}$, where $F_{\varepsilon}=\left(X^{\varepsilon}(1, x, w)-\right.$ $x) / \varepsilon$ is uniformly non-degenerate), we have

$$
\begin{aligned}
& \left\|\left(\partial_{k} f\right)\left(X_{t}\right)\right\|_{p}^{p^{\prime}}=\left\|\left(\partial_{k} f\right)\left(x+\varepsilon F_{\varepsilon}\right)\right\|_{p}^{p^{\prime}} \leqslant c_{1}\left\|\left(\partial_{k} f\right)(x+\varepsilon z)\right\|_{L_{p^{\prime}}\left(\mathbb{R}^{d}, \mathrm{~d} z\right)}^{p^{\prime}} \\
& \leqslant c_{1}^{\prime} \int_{\mathbb{R}^{d}}\left\{\int_{0}^{\infty} u^{-1 / 2} \mathrm{e}^{-u}(2 \pi c u)^{-d / 2} \exp \left\{-\frac{|\varepsilon z-(y-x)|^{2}}{2 c u}\right\} \mathrm{d} u\right\}^{p^{\prime}} \mathrm{d} z \\
& =c_{1}^{\prime} \varepsilon^{-d} \int_{\mathbb{R}^{d}}\left\{|z-(y-x)|^{(1-d)} \int_{0}^{\infty} v^{\frac{d-1}{2}-1} \mathrm{e}^{-v} \mathrm{e}^{-\frac{|z-(y-x)|^{2}}{2 c v}} \mathrm{~d} v\right\}^{p^{\prime}} \mathrm{d} z .
\end{aligned}
$$

for some constants $c_{1}, c_{1}^{\prime}>0$ independent of $\varepsilon$ (In the last equality, we have applied the change of variables $\left.v=\frac{|\varepsilon z-(y-x)|^{2}}{2 c u}\right)$. The last factor can be further computed as follows:

$$
\begin{aligned}
& \int_{\mathbb{R}^{d}}\left\{|z|^{(1-d)} \int_{0}^{\infty} v^{\frac{d-1}{2}-1} \mathrm{e}^{-v} \mathrm{e}^{-\frac{|z|^{2}}{2 c v}} \mathrm{~d} v\right\}^{p^{\prime}} \mathrm{d} z \\
& \leqslant \int_{|z|<1}\left\{|z|^{(1-d)} \int_{0}^{\infty} v^{\frac{d-1}{2}-1} \mathrm{e}^{-v} \mathrm{~d} v\right\}^{p^{\prime}} \mathrm{d} z \\
& \quad+\int_{|z| \geqslant 1}\left\{|z|^{(1-d)} \int_{0}^{\infty} v^{\frac{d-1}{2}-1} \mathrm{e}^{-\frac{(|z|-\sqrt{2 c} v)^{2}}{2 c v}} \mathrm{e}^{-\sqrt{(2 / c)}|z|} \mathrm{d} v\right\}^{p^{\prime}} \mathrm{d} z .
\end{aligned}
$$

(For getting the second term, note that $\mathrm{e}^{-v} \mathrm{e}^{-|z|^{2} /(2 c v)}=\mathrm{e}^{-\frac{(|z|-\sqrt{2 c v})^{2}}{2 c v}} \mathrm{e}^{-\sqrt{(2 / c)}|z|}$.) The first term equals to $\left(\int_{|z|<1}|z|^{p^{\prime}(1-d)} \mathrm{d} z\right)\left(\int_{0}^{\infty} v^{\frac{d-1}{2}-1} \mathrm{e}^{-v} \mathrm{~d} v\right)^{p^{\prime}}$. The assumption $d \geqslant 2$ assures $\int_{0}^{\infty} v^{\frac{d-1}{2}-1} \mathrm{e}^{-v} \mathrm{~d} v<+\infty$ and hence the first term is finite if $p^{\prime}<\frac{d}{d-1}$. For the second term, we have

$$
\int_{|z| \geqslant 1}\left\{|z|^{(1-d)} \int_{0}^{\infty} v^{\frac{d-1}{2}-1} \mathrm{e}^{-\frac{(|z|-\sqrt{2 c} v)^{2}}{2 c v}} \mathrm{e}^{-\sqrt{(2 / c)}|z|} \mathrm{d} v\right\}^{p^{\prime}} \mathrm{d} z \leqslant c_{1}^{\prime \prime}(I+I I),
$$

where $c_{1}^{\prime \prime}>0$ is a constant depending only on $p^{\prime}$, and $I, I I$ are defined by

$$
\begin{aligned}
& I:=\int_{|z| \geqslant 1}\left\{|z|^{(1-d)} \mathrm{e}^{-\sqrt{(2 / c)}|z|} \int_{0}^{|z|} v^{\frac{d-1}{2}-1} \mathrm{~d} v\right\}^{p^{\prime}} \mathrm{d} z \\
& I I:=\int_{|z| \geqslant 1}\left\{|z|^{(1-d)} \mathrm{e}^{-\sqrt{(2 / c)}|z|} \int_{|z|}^{\infty} v^{\frac{d-1}{2}-1} \mathrm{e}^{-\frac{(|z|-\sqrt{2 c} v)^{2}}{2 c v}} \mathrm{~d} v\right\}^{p^{\prime}} \mathrm{d} z
\end{aligned}
$$

The term $I$ is estimated as $I=\left(\frac{2}{d-1}\right)^{p^{\prime}} \int_{|z| \geqslant 1}\left\{|z|^{-\frac{d-1}{2}} \mathrm{e}^{-\sqrt{(2 / c)}|z|}\right\}^{p^{\prime}} \mathrm{d} z \leqslant$ $\left(\frac{2}{d-1}\right)^{p^{\prime}} \int_{|z| \geqslant 1} \mathrm{e}^{-p^{\prime} \sqrt{(2 / c)}|z|} \mathrm{d} z<+\infty$. On the other hand, by applying the change of 
variable $v=|z| u$ and noting that $|z| \geqslant 1$,

$$
\begin{aligned}
I I & =\int_{|z| \geqslant 1}\left\{|z|^{-\frac{d-1}{2}} \mathrm{e}^{-\sqrt{(2 / c)}|z|} \int_{1}^{\infty} u^{\frac{d-1}{2}-1} \mathrm{e}^{-\frac{|z|(1-\sqrt{2 c} u)^{2}}{2 c u}} \mathrm{~d} u\right\}^{p^{\prime}} \mathrm{d} z \\
& \leqslant \int_{|z| \geqslant 1} \mathrm{e}^{-p^{\prime} \sqrt{(2 / c)}|z|}\left\{\int_{1}^{\infty} u^{\frac{d-1}{2}-1} \mathrm{e}^{-\frac{(1-\sqrt{2 c} u)^{2}}{2 c u}} \mathrm{~d} u\right\}^{p^{\prime}} \mathrm{d} z .
\end{aligned}
$$

Since $\exp \left(-\frac{(1-\sqrt{2 c u})^{2}}{2 c u}\right)=\mathrm{e}^{-\frac{1}{2 c u}} \mathrm{e}^{\sqrt{2 / c}} \exp (-u) \leqslant \mathrm{e}^{\sqrt{2 / c}} \exp (-u)$, we get

$$
I I \leqslant \mathrm{e}^{p^{\prime} \sqrt{2 / c}}\left(\int_{|z| \geqslant 1} \mathrm{e}^{-p^{\prime} \sqrt{2 / c}|z|} \mathrm{d} z\right)\left(\int_{1}^{\infty} u^{\frac{d-1}{2}-1} \mathrm{e}^{-u} \mathrm{~d} u\right)^{p^{\prime}}<+\infty
$$

(the arguments so far will be used repeatedly below). Putting all together, we have obtained that for $p^{\prime} \in\left(p, \frac{d}{d-1}\right)$, $\lim _{\sup _{\varepsilon \downarrow 0}} \varepsilon^{d}\left\|\left(\partial_{k} f\right)\left(X_{t}\right)\right\|_{p}^{p^{\prime}}<+\infty$, which implies (A.8).

For (A.9), with assuming $\varepsilon \in(0,1]$, we have

$$
\begin{aligned}
& \left|(1-\triangle)^{s / 2}\left[\left(\phi \partial_{k} f\right)(x+\varepsilon \bullet)\right](z)\right| \\
& =\left|\frac{1}{\Gamma\left(-\frac{s}{2}\right)} \int_{0}^{\infty} u^{-\frac{s}{2}-1} \mathrm{e}^{-u} \int_{\mathbb{R}^{d}} \frac{\mathrm{e}^{-\frac{\left|z-z^{\prime}\right|^{2}}{4 u}}}{(4 \pi u)^{d / 2}}\left(\phi \partial_{k} f\right)\left(x+\varepsilon z^{\prime}\right) \mathrm{d} z^{\prime} \mathrm{d} u\right|
\end{aligned}
$$

By using (A.4), change of variables $\varepsilon z^{\prime}=z^{\prime \prime}$ and the semigroup property of $\mathrm{e}^{t \triangle / 2}$ (recall that $c \geqslant 2$ ), we have

$$
\begin{aligned}
& \int_{\mathbb{R}^{d}} \frac{\mathrm{e}^{-\frac{\left|z-z^{\prime}\right|^{2}}{4 u}}}{(4 \pi u)^{d / 2}}\left|\left(\phi \partial_{k} f\right)\left(x+\varepsilon z^{\prime}\right)\right| \mathrm{d} z^{\prime} \\
& \leqslant C \int_{0}^{+\infty} v^{-1 / 2} \mathrm{e}^{-v} \mathrm{~d} v \int_{\mathbb{R}^{d}} \frac{\mathrm{e}^{-\frac{\left|z-z^{\prime}\right|^{2}}{4 u}}}{(4 \pi u)^{d / 2}} \frac{\mathrm{e}^{-\frac{\left|\left(x+\varepsilon z^{\prime}\right)-y\right|^{2}}{2 c v}}}{(2 \pi c v)^{d / 2}} \mathrm{~d} z^{\prime} \\
& =C \varepsilon^{d} \int_{0}^{+\infty} v^{-1 / 2} \mathrm{e}^{-v} \mathrm{~d} v \int_{\mathbb{R}^{d}} \frac{\mathrm{e}^{-\frac{\left|\varepsilon z-\varepsilon z^{\prime}\right|^{2}}{4 \varepsilon^{2} u}}}{\left(4 \pi \varepsilon^{2} u\right)^{d / 2}} \frac{\mathrm{e}^{-\frac{\left|\left(x+\varepsilon z^{\prime}\right)-y\right|^{2}}{2 c v}}}{(2 \pi c v)^{d / 2}} \mathrm{~d} z^{\prime} \\
& =C \int_{0}^{+\infty} v^{-1 / 2} \mathrm{e}^{-v} \mathrm{~d} v \int_{\mathbb{R}^{d}} \frac{\mathrm{e}^{-\frac{\left|\varepsilon z-z^{\prime \prime}\right|^{2}}{4 \varepsilon^{2} u}}}{\left(4 \pi \varepsilon^{2} u\right)^{d / 2}} \frac{\mathrm{e}^{-\frac{\left|\left(x+z^{\prime \prime}\right)-y\right|^{2}}{2 c v}}}{(2 \pi c v)^{d / 2}} \mathrm{~d} z^{\prime \prime} \\
& =C \int_{0}^{+\infty} v^{-1 / 2} \mathrm{e}^{-v} \frac{\mathrm{e}^{-\frac{|\varepsilon z-(y-x)|^{2}}{2\left(2 \varepsilon^{2} u+c v\right)}}}{\left(2 \pi\left(2 \varepsilon^{2} u+c v\right)\right)^{d / 2}} \mathrm{~d} v \\
& \leqslant C\left(2^{-1} c\right)^{d / 2} \int_{0}^{+\infty} v^{-1 / 2} \mathrm{e}^{-v} \frac{\mathrm{e}^{-\frac{|\varepsilon z-(y-x)|^{2}}{2\left(c \varepsilon^{2} u+c v\right)}}}{\left(2 \pi\left(c \varepsilon^{2} u+c v\right)\right)^{d / 2}} \mathrm{~d} v,
\end{aligned}
$$

where in the last inequality, we have used that

$$
\sup _{u, v \geqslant 0} \frac{\left(c \varepsilon^{2}+c v\right)^{d / 2}}{\left(2 \varepsilon^{2}+c v\right)^{d / 2}} \leqslant \sup _{u, v \geqslant 0} \frac{\left(c \varepsilon^{2}+c v\right)^{d / 2}}{\left(2 \varepsilon^{2}+2 v\right)^{d / 2}}=\left(2^{-1} c\right)^{d / 2} .
$$


Substituting this estimate into (A.12), we get

$$
\begin{aligned}
& \left|(1-\triangle)^{s / 2}\left[\left(\phi \partial_{k} f\right)(x+\varepsilon \bullet)\right](z)\right| \\
& \leqslant c_{2} \int_{0}^{\infty} \int_{0}^{\infty} u^{-\frac{s}{2}-1} \mathrm{e}^{-u} v^{-1 / 2} \mathrm{e}^{-v} \frac{\mathrm{e}^{-\frac{|\varepsilon z-(y-x)|^{2}}{2 c\left(\varepsilon^{2} u+v\right)}}}{\left(2 \pi c\left(\varepsilon^{2} u+v\right)\right)^{d / 2}} \mathrm{~d} u \mathrm{~d} v \\
& =c_{2} \varepsilon^{s} \int_{0}^{\infty} \int_{0}^{\infty} u^{-\frac{s}{2}-1} v^{-1 / 2} \mathrm{e}^{-\left(\varepsilon^{-2} u+v\right)} \frac{\mathrm{e}^{-\frac{|\varepsilon z-(y-x)|^{2}}{2 c(u+v)}}}{(2 \pi c(u+v))^{d / 2}} \mathrm{~d} u \mathrm{~d} v \\
& \leqslant c_{2} \varepsilon^{s} \int_{0}^{\infty} \int_{0}^{\infty} u^{-\frac{s}{2}-1} v^{-1 / 2} \mathrm{e}^{-(u+v)} \frac{\mathrm{e}^{-\frac{|\varepsilon z-(y-x)|^{2}}{2 c(u+v)}}}{(2 \pi c(u+v))^{d / 2}} \mathrm{~d} u \mathrm{~d} v \\
& =c_{2}^{\prime} \varepsilon^{s} \int_{0}^{\infty} u^{\frac{1-s}{2}-1} \mathrm{e}^{-u} \frac{\mathrm{e}^{-\frac{|\varepsilon z-(y-x)|^{2}}{2 c u}}}{(2 \pi c u)^{d / 2}} \mathrm{~d} u,
\end{aligned}
$$

for some constants $c_{2}, c_{2}^{\prime}>0$ independent of $\varepsilon$, and so that, by using Lemma 4.12,

$$
\begin{aligned}
& \left\|(I-\mathcal{L})^{s / 2}\left(\phi \partial_{k} f\right)\left(X_{t}\right)\right\|_{p}^{p^{\prime}} \\
& \leqslant c_{2}^{\prime \prime}\left\|(1-\triangle)^{s / 2}\left[\left(\phi \partial_{k} f\right)(x+\varepsilon \bullet)\right](z)\right\|_{L_{p^{\prime}}\left(\mathbb{R}^{d}, \mathrm{~d} z\right)}^{p^{\prime}} \\
& \leqslant c_{2}^{\prime \prime \prime} \varepsilon^{s p^{\prime}} \int_{\mathbb{R}^{d}}\left\{\int_{0}^{\infty} u^{\frac{1-s}{2}-1} \mathrm{e}^{-u} \frac{\mathrm{e}^{-\frac{|\varepsilon z-(y-x)|^{2}}{2 \pi u}}}{(2 \pi c u)^{d / 2}} \mathrm{~d} u\right\}^{p^{\prime}} \mathrm{d} z \\
& =c_{2}^{\prime \prime \prime} \varepsilon^{s p^{\prime}-d} \int_{\mathbb{R}^{d}}\left\{|z-(y-x)|^{-(d-(1-s))}\right. \\
& \left.\quad \times \int_{0}^{\infty} u^{\frac{d-(1-s)}{2}-1} \mathrm{e}^{-u} \mathrm{e}^{-\frac{|z-(y-x)|^{2}}{2 c u}} \mathrm{~d} u\right\}^{p^{\prime}} \mathrm{d} z .
\end{aligned}
$$

for some constants $c_{2}^{\prime \prime}, c_{2}^{\prime \prime \prime}>0$. Here, note that $d-(1-s)>0$ because $d \geqslant 2$ and $s>-\frac{d}{2}$. Hence $\int_{0}^{\infty} u^{\frac{d-(1-s)}{2}-1} \mathrm{e}^{-u} \mathrm{~d} u<+\infty$ and by repeating the above argument, we see that if $p<p^{\prime}<\frac{d}{d+s-1}$, limsup $\sup _{\varepsilon \downarrow 0} \varepsilon^{-\left(s p^{\prime}-d\right)}\left\|(I-\mathcal{L})^{s / 2}\left(\phi \partial_{k} f\right)\left(X_{t}\right)\right\|_{p}^{p^{\prime}}<+\infty$, so that

$$
\begin{aligned}
& \limsup _{\varepsilon \downarrow 0}\left(\varepsilon^{-(s p-d)}\left\|(I-\mathcal{L})^{s / 2}\left(\phi \partial_{k} f\right)\left(X_{t}\right)\right\|_{p}^{p}\right)^{p^{\prime} / p} \\
& =\underset{\varepsilon \downarrow 0}{\limsup } \varepsilon^{d\left(\frac{p^{\prime}}{p}-1\right)}\left(\varepsilon^{-\left(s p^{\prime}-d\right)}\left\|(I-\mathcal{L})^{s / 2}\left(\phi \partial_{k} f\right)\left(X_{t}\right)\right\|_{p}^{p^{\prime}}\right)=0 .
\end{aligned}
$$

This is nothing but (A.9).

Next we prove (A.10). By virtue of Lemma 4.12, it suffices to show

$$
\begin{aligned}
& \|f(x+\varepsilon z)\|_{L_{p^{\prime}}\left(\mathbb{R}^{d}, \mathrm{~d} z\right)}^{p^{\prime}} \\
& \leqslant c_{3} \varepsilon^{-d}\left[\int_{\mathbb{R}^{d}}\left\{|z-(x-y)|^{1-d} \int_{0}^{\infty} u^{\frac{d}{2}-1} \mathrm{e}^{-u} \mathrm{e}^{-\frac{|z-(x-y)|^{2}}{2 c u}} \mathrm{~d} u\right\}^{p^{\prime}} \mathrm{d} z\right. \\
& \left.\quad+\int_{\mathbb{R}^{d}}\left\{|z-(x-y)|^{-(d-1) / 2} \int_{0}^{\infty} u^{-1 / 2} \mathrm{e}^{-u} \mathrm{e}^{-\frac{|z-(x-y)|^{2}}{2 c u}} \mathrm{~d} u\right\}^{p^{\prime}} \mathrm{d} z\right]
\end{aligned}
$$

for some constant $c_{3}>0$, independent of $\varepsilon$. Actually, then we have $\lim \sup _{\varepsilon \downarrow 0} \varepsilon^{d}\left\|f\left(X_{t}\right)\right\|_{p}^{p^{\prime}}<+\infty$ if $p<p^{\prime}<\frac{d}{d-1}$, from which we can conclude (A.10). 
To prove (A.13), we begin with the inequality

$$
f(x+\varepsilon z) \leqslant \frac{C(2 \pi c)^{-d / 2}}{\Gamma(1)} \int_{0}^{\infty} u^{-d / 2} \mathrm{e}^{-u} \mathrm{e}^{-\frac{|y-(x+\varepsilon z)|^{2}}{2 c u}} \mathrm{~d} u .
$$

We divide the integral as

$$
\begin{aligned}
& \int_{0}^{\infty} u^{-d / 2} \mathrm{e}^{-u} \mathrm{e}^{-\frac{|y-(x+\varepsilon z)|^{2}}{2 c u}} \mathrm{~d} u \\
& =\int_{0}^{|y-(x+\varepsilon z)|} u^{-d / 2} \mathrm{e}^{-u} \mathrm{e}^{-\frac{|y-(x+\varepsilon z)|^{2}}{2 c u}} \mathrm{~d} u+\int_{|y-(x+\varepsilon z)|}^{\infty} u^{-d / 2} \mathrm{e}^{-u} \mathrm{e}^{-\frac{|y-(x+\varepsilon z)|^{2}}{2 c u}} \mathrm{~d} u .
\end{aligned}
$$

The first term in the last equation is estimated as

$$
\begin{aligned}
& \int_{0}^{|y-(x+\varepsilon z)|} u^{-d / 2} \mathrm{e}^{-u} \mathrm{e}^{-\frac{|\varepsilon z-(x-y)|^{2}}{2 c u}} \mathrm{~d} u \\
& =(2 c)^{\frac{d}{2}-1}|\varepsilon z-(x-y)|^{2-d} \int_{\frac{|y-(x+\varepsilon z)|}{2 c}}^{\infty} u^{\frac{d}{2}-2} \mathrm{e}^{-u} \mathrm{e}^{-\frac{|\varepsilon z-(x-y)|^{2}}{2 c u}} \mathrm{~d} u \\
& \leqslant(2 c)^{\frac{d}{2}}|\varepsilon z-(x-y)|^{1-d} \int_{\frac{|y-(x+\varepsilon z)|}{2 c}}^{\infty} u^{\frac{d}{2}-1} \mathrm{e}^{-u} \mathrm{e}^{-\frac{|\varepsilon z-(x-y)|^{2}}{2 c u}} \mathrm{~d} u \\
& \leqslant(2 c)^{\frac{d}{2}}|\varepsilon z-(x-y)|^{1-d} \int_{0}^{\infty} u^{\frac{d}{2}-1} \mathrm{e}^{-u} \mathrm{e}^{-\frac{|\varepsilon z-(x-y)|^{2}}{2 c u}} \mathrm{~d} u .
\end{aligned}
$$

On the other hand, the second term is

$$
\begin{aligned}
& \int_{|y-(x+\varepsilon z)|}^{\infty} u^{-d / 2} \mathrm{e}^{-u} \mathrm{e}^{-\frac{|y-(x+\varepsilon z)|^{2}}{2 c u}} \mathrm{~d} u \\
& \leqslant|\varepsilon z-(x-y)|^{-(d-1) / 2} \int_{0}^{\infty} u^{-1 / 2} \mathrm{e}^{-u} \mathrm{e}^{-\frac{|y-(x+\varepsilon z)|^{2}}{2 c u}} \mathrm{~d} u .
\end{aligned}
$$

Now a change of variable leads us to (A.13) and thus (A.10) is proved.

For (A.11), it is sufficient to prove

$$
\begin{aligned}
& \left\|(1-\triangle)^{s / 2}[(\phi f)(x+\varepsilon \bullet)](z)\right\|_{L_{p^{\prime}}\left(\mathbb{R}^{d}, \mathrm{~d} z\right)}^{p^{\prime}} \\
& \leqslant c_{4} \varepsilon^{s p^{\prime}-d}\left[\int_{\mathbb{R}^{d}}\left\{|z-(x-y)|^{1-(s+d)} \int_{0}^{\infty} u^{\frac{d+s}{2}-1} \mathrm{e}^{-u} \mathrm{e}^{-\frac{|z-(x-y)|^{2}}{2 c u}} \mathrm{~d} u\right\}^{p^{\prime}} \mathrm{d} z\right. \\
& \left.\quad+\int_{\mathbb{R}^{d}}\left\{|z-(x-y)|^{\frac{2-d}{2}} \int_{0}^{\infty} u^{-\frac{s}{2}} \mathrm{e}^{-u} \mathrm{e}^{-\frac{|z-(x-y)|^{2}}{2 c u}} \mathrm{~d} u\right\}^{p^{\prime}} \mathrm{d} z\right]
\end{aligned}
$$

where $c_{4}>0$ is a constant independent of $\varepsilon$. Note that $d+s>0$ and $-s>0$, so that it is assured that $\int_{0}^{\infty} u^{\frac{d+s}{2}-1} \mathrm{e}^{-u} \mathrm{~d} u<+\infty$ and $\int_{0}^{\infty} u^{-\frac{s}{2}} \mathrm{e}^{-u} \mathrm{~d} u<+\infty$, respectively. Note also that $1-(s+d)<0$. These imply $\lim \sup _{\varepsilon \downarrow 0} \varepsilon^{d-s p^{\prime}}\left\|(I-\mathcal{L})^{s / 2}(\phi f)\left(X_{t}\right)\right\|_{p}^{p^{\prime}}<$ $+\infty$ if $\left.p<p^{\prime}<\min \left\{\frac{d}{d+s-1}, \frac{2 d}{d-2}\right\}\right\}$, and so (A.11).

To prove (A.14), we apply a similar argument, with assuming $\varepsilon \in(0,1]$, which leads to

$$
\begin{aligned}
& \left|(1-\triangle)^{s / 2}[(\phi f)(x+\varepsilon \bullet)](z)\right| \\
& \leqslant \text { const. } \varepsilon^{s} \int_{0}^{\infty} u^{\frac{2-(s+d)}{2}}-1 \mathrm{e}^{-u} \mathrm{e}^{-\frac{|\varepsilon z-(x-y)|^{2}}{2 c u}} \mathrm{~d} u
\end{aligned}
$$


We divide the integral as

$$
\begin{aligned}
& \int_{0}^{\infty} u^{\frac{2-(s+d)}{2}-1} \mathrm{e}^{-u} \mathrm{e}^{-\frac{|\varepsilon z-(x-y)|^{2}}{2 c u}} \mathrm{~d} u \\
& =\int_{0}^{|\varepsilon z-(x-y)|} u^{\frac{2-(s+d)}{2}-1} \mathrm{e}^{-u} \mathrm{e}^{-\frac{|\varepsilon z-(x-y)|^{2}}{2 c u}} \mathrm{~d} u \\
& \quad+\int_{|\varepsilon z-(x-y)|}^{\infty} u^{\frac{2-(s+d)}{2}-1} \mathrm{e}^{-u} \mathrm{e}^{-\frac{|\varepsilon z-(x-y)|^{2}}{2 c u}} \mathrm{~d} u .
\end{aligned}
$$

We estimate the first term as follows.

$$
\begin{aligned}
& \int_{0}^{|\varepsilon z-(x-y)|} u^{\frac{2-(s+d)}{2}-1} \mathrm{e}^{-u} \mathrm{e}^{-\frac{|\varepsilon z-(x-y)|^{2}}{2 c u}} \mathrm{~d} u \\
& =(2 c)^{1-\frac{d+s-2}{2}}|\varepsilon z-(x-y)|^{2-(s+d)} \int_{\frac{|\varepsilon z-(x-y)|}{2 c}}^{\infty} u^{\frac{d+s-2}{2}-1} \mathrm{e}^{-u} \mathrm{e}^{-\frac{|\varepsilon z-(x-y)|^{2}}{2 c u}} \mathrm{~d} u \\
& \leqslant(2 c)^{1-\frac{d+s-3}{2}}|\varepsilon z-(x-y)|^{1-(s+d)} \int_{\frac{|\varepsilon z-(x-y)|}{2 c}}^{\infty} u^{\frac{d+s}{2}-1} \mathrm{e}^{-u} \mathrm{e}^{-\frac{|\varepsilon z-(x-y)|^{2}}{2 c u}} \mathrm{~d} u \\
& \leqslant(2 c)^{1-\frac{d+s-3}{2}}|\varepsilon z-(x-y)|^{1-(s+d)} \int_{0}^{\infty} u^{\frac{d+s}{2}-1} \mathrm{e}^{-u} \mathrm{e}^{-\frac{|\varepsilon z-(x-y)|^{2}}{2 c u}} \mathrm{~d} u .
\end{aligned}
$$

The second term is estimated as

$$
\begin{aligned}
& \int_{|\varepsilon z-(x-y)|}^{\infty} u^{\frac{2-(s+d)}{2}-1} \mathrm{e}^{-u} \mathrm{e}^{-\frac{|\varepsilon z-(x-y)|^{2}}{2 c u}} \mathrm{~d} u \\
& \leqslant|\varepsilon z-(x-y)|^{\frac{2-d}{2}} \int_{0}^{\infty} u^{-\frac{s}{2}} \mathrm{e}^{-u} \mathrm{e}^{-\frac{|\varepsilon z-(x-y)|^{2}}{2 c u}} \mathrm{~d} u .
\end{aligned}
$$

Combining these, and a change of variable, we reach the estimate (A.14), and hence (A.11) is proved.

(d) In view of (A.8), (A.9), (A.10) and (A.11), what we have to do now is to find $p^{\prime}, q, r>1$ such that

- $\frac{1}{p^{\prime}}+\frac{1}{q}+\frac{1}{r}<1$;

- $p^{\prime}<\frac{d}{d-1}$ and $p^{\prime \prime}:=q(p-1)<\frac{d}{d-(1-s)}$.

In fact, since $s<\frac{p}{p-1}-d$, we can take $\varepsilon \in\left(0, \frac{1}{d}\right)$ such that $s<\frac{p}{p-1}-\frac{d}{1-\varepsilon d}<\frac{p}{p-1}-d$. Then take $p^{\prime}, q>1$ so that

$$
\frac{d-1}{d}<\frac{1}{p^{\prime}}<\frac{d-1}{d}+\varepsilon \text { and } \frac{1}{q}=\frac{1}{d}-\varepsilon\left(<\frac{1}{d}\right) .
$$

These conditions imply $1 / p^{\prime}+1 / q<\frac{d-1}{d}+\frac{1}{d}=1$ and hence one can take $r>0$ such that $1 / p^{\prime}+1 / q+1 / r<1$. Finally, we have if $p^{\prime \prime}-1>0$, then $1-\frac{\left(p^{\prime \prime}-1\right) d}{p^{\prime \prime}}=$ $1-\frac{\left(p^{\prime \prime}-1\right) d}{(p-1) q}>1-\frac{\left(p^{\prime \prime}-1\right) d}{(p-1) d}=1-\frac{p^{\prime \prime}-1}{p-1}=\frac{p}{p-1}-q=\frac{p}{p-1}-\frac{d}{1-\varepsilon d}>s$, which implies $p^{\prime \prime}<\frac{d}{d-(1-s)}$. If $p^{\prime \prime}-1 \leqslant 0$, then $1-\frac{\left(p^{\prime \prime}-1\right) d}{p^{\prime \prime}}>0>s$, which also implies $p^{\prime \prime}<\frac{d}{d-(1-s)}$.

Therefore, by taking $p^{\prime}, q, r>1$ as above, we conclude from (A.5), (A.6) and (A.7)-(A.11) that $\left\|\left(\phi \partial_{k} f\right)\left(X_{t}\right)\right\|_{p, s}$ and $\left\|(\phi f)\left(X_{t}\right)\right\|_{p, s}$ converge to zero as $t=\varepsilon^{2} \downarrow 0$. 


\section{Acknowledgements}

The author would like to thank an anonymous referee for pointing out several mistakes in a preliminary version.

\section{References}

H. Abels. Pseudodifferential and singular integral operators. De Gruyter Graduate Lectures. De Gruyter, Berlin (2012). ISBN 978-3-11-025030-5. MR2884718.

H. Airault, J. Ren and X. Zhang. Smoothness of local times of semimartingales. C. R. Acad. Sci. Paris Sér. I Math. 330 (8), 719-724 (2000). MR1763917.

M. Ait Ouahra, A. Kissami and H. Ouahhabi. On fractional derivatives of the local time of a symmetric stable process as a doubly indexed process. Random Oper. Stoch. Equ. 22 (2), 99-107 (2014). MR3213598.

C. Bender. An Itô formula for generalized functionals of a fractional Brownian motion with arbitrary Hurst parameter. Stochastic Process. Appl. 104 (1), 81106 (2003). MR1956473.

B. Bongioanni and J. L. Torrea. Sobolev spaces associated to the harmonic oscillator. Proc. Indian Acad. Sci. Math. Sci. 116 (3), 337-360 (2006). MR2256010.

B. Boufoussi and B. Roynette. Le temps local brownien appartient p.s. à l'espace de Besov $\mathscr{B}_{p, \infty}^{1 / 2}$. C. R. Acad. Sci. Paris Sér. I Math. 316 (8), 843-848 (1993). MR1218273.

N. Bouleau and M. Yor. Sur la variation quadratique des temps locaux de certaines semimartingales. C. R. Acad. Sci. Paris Sér. I Math. 292 (9), 491-494 (1981). MR612544.

H. Föllmer, P. Protter and A. N. Shiryayev. Quadratic covariation and an extension of Itô's formula. Bernoulli 1 (1-2), 149-169 (1995). MR1354459.

A. Friedman. Partial differential equations of parabolic type. Prentice-Hall, Inc., Englewood Cliffs, N.J. (1964). MR0181836.

M. Hasumi. Note on the $n$-dimensional tempered ultra-distributions. Tôhoku Math. J. (2) 13, 94-104 (1961). MR0131759.

N. Ikeda and S. Watanabe. Stochastic differential equations and diffusion processes, volume 24 of North-Holland Mathematical Library. North-Holland Publishing Co., Amsterdam; Kodansha, Ltd., Tokyo, second edition (1989). ISBN 0-44487378-3. MR1011252.

N. V. Krylov. Lectures on elliptic and parabolic equations in Sobolev spaces, volume 96 of Graduate Studies in Mathematics. American Mathematical Society, Providence, RI (2008). ISBN 978-0-8218-4684-1. MR2435520.

I. Kubo. Itô formula for generalized Brownian functionals. In Theory and application of random fields (Bangalore, 1982), volume 49 of Lect. Notes Control Inf. Sci., pages 156-166. Springer, Berlin (1983). MR799940.

S. Kusuoka and D. Stroock. Applications of the Malliavin calculus. II. J. Fac. Sci. Univ. Tokyo Sect. IA Math. 32 (1), 1-76 (1985). MR783181.

Z. Liang. Fractional smoothness for the generalized local time of the indefinite Skorohod integral. J. Funct. Anal. 239 (1), 247-267 (2006). MR2258223.

S. Lou and C. Ouyang. Local times of stochastic differential equations driven by fractional Brownian motions. Stochastic Process. Appl. 127 (11), 3643-3660 (2017). MR3707240. 
Distributional Itô's Formula and Regularization of Generalized Wiener Functionals 753

D. Nualart and J. Vives. Chaos expansions and local times. Publ. Mat. 36 (2B), 827-836 (1993) (1992). MR1210022.

D. Revuz and M. Yor. Continuous martingales and Brownian motion, volume 293 of Grundlehren der Mathematischen Wissenschaften [Fundamental Principles of Mathematical Sciences]. Springer-Verlag, Berlin, third edition (1999). ISBN 3540-64325-7. MR1725357.

J. Sebastião e Silva. Les fonctions analytiques comme ultra-distributions dans le calcul opérationnel. Math. Ann. 136, 58-96 (1958). MR0105615.

E. M. Stein. Interpolation of linear operators. Trans. Amer. Math. Soc. 83, 482-492 (1956). MR0082586.

S. Thangavelu. Lectures on Hermite and Laguerre expansions, volume 42 of Mathematical Notes. Princeton University Press, Princeton, NJ (1993). ISBN 0-69100048-4. MR1215939.

H. F. Trotter. A property of Brownian motion paths. Illinois J. Math. 2, 425-433 (1958). MR0096311.

H. Uemura. Tanaka formula for multidimensional Brownian motions. J. Theoret. Probab. 17 (2), 347-366 (2004). MR2053708.

A. Ju. Veretennikov and N. V. Krylov. Explicit formulae for the solutions of stochastic equations. Mat. Sb. (N.S.) 100(142) (2), 266-284, 336 (1976). MR0410921.

A. T. Wang. Generalized Ito's formula and additive functionals of Brownian motion. Z. Wahrscheinlichkeitstheorie und Verw. Gebiete 41 (2), 153-159 (1977/78). MR0488327.

S. Watanabe. Analysis of Wiener functionals (Malliavin calculus) and its applications to heat kernels. Ann. Probab. 15 (1), 1-39 (1987). MR877589.

S. Watanabe. Donsker's $\delta$-functions in the Malliavin calculus. In Stochastic analysis, pages 495-502. Academic Press, Boston, MA (1991). MR1119846.

S. Watanabe. Fractional order Sobolev spaces on Wiener space. Probab. Theory Related Fields 95 (2), 175-198 (1993). MR1214086.

S. Watanabe. Some refinements of Donsker's delta functions. In Stochastic analysis on infinite-dimensional spaces (Baton Rouge, LA, 1994), volume 310 of Pitman Res. Notes Math. Ser., pages 308-324. Longman Sci. Tech., Harlow (1994a). MR1415679.

S. Watanabe. Stochastic Levi sums. Comm. Pure Appl. Math. 47 (5), 767-786 (1994b). MR1278353.

K. Yoshinaga. On spaces of distributions of exponential growth. Bull. Kyushu Inst. Tech. Math. Nat. Sci. 6, 1-16 (1960). MR0126717. 